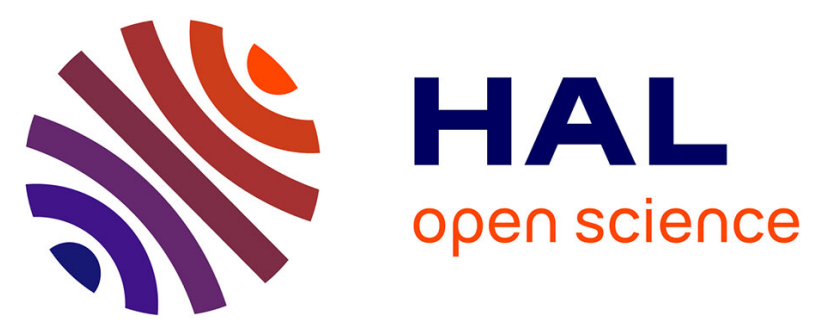

\title{
Premiers temps du Magdalénien en Gironde: réévaluation des fouilles Trécolle à Saint-Germain-la-Rivière (France)
}

Mathieu Langlais, Véronique Laroulandie, Sandrine Costamagno, Jean-Marc

Pétillon, Jean-Baptiste Mallye, François Lacrampe-Cuyaubère, Myriam

Boudadi-Maligne, Carolyn Barshay-Szmidt, Caroline Masset, Éric Pubert, et al.

\section{- To cite this version:}

Mathieu Langlais, Véronique Laroulandie, Sandrine Costamagno, Jean-Marc Pétillon, Jean-Baptiste Mallye, et al.. Premiers temps du Magdalénien en Gironde : réévaluation des fouilles Trécolle à SaintGermain-la-Rivière (France). Bulletin de la Société préhistorique française, 2015, 112 (1), pp.5-58. 10.3406/bspf.2015.14489 . hal-01891673

\section{HAL Id: hal-01891673 https://hal.science/hal-01891673}

Submitted on 23 Oct 2018

HAL is a multi-disciplinary open access archive for the deposit and dissemination of scientific research documents, whether they are published or not. The documents may come from teaching and research institutions in France or abroad, or from public or private research centers.
L'archive ouverte pluridisciplinaire HAL, est destinée au dépôt et à la diffusion de documents scientifiques de niveau recherche, publiés ou non, émanant des établissements d'enseignement et de recherche français ou étrangers, des laboratoires publics ou privés. 


\title{
Premiers temps du Magdalénien en Gironde
}

\author{
Réévaluation des fouilles Trécolle \\ à Saint-Germain-la-Rivière (France)
}

\author{
Mathieu Langlais, Véronique Laroulandie, Sandrine Costamagno, Jean-Marc Pétillon, \\ Jean-Baptiste Mallye, François Lacrampe-Cuyaubère, Myriam Boudadi-Maligne, \\ Carolyn Barshay-Szmidt, Caroline Masset, Éric Pubert, William Rendu et Michel Lenoir
}

Résumé : Le site magdalénien de Saint-Germain-la-Rivière (Gironde, France) a régulièrement été sollicité dans le cadre de plusieurs débats concernant le Paléolithique supérieur récent d'Europe de l'Ouest. L'essentiel de ces travaux s'appuient sur l'analyse, d'une part, du matériel mis au jour par R. Blanchard - notamment la célèbre sépulture - et, d'autre part, de celui provenant des fouilles plus méthodiques conduites par G. Trécolle dans le talus. À la suite de l'étude de l'ensemble des collections menée par M. Lenoir qui a permis d'attribuer les différentes occupations du gisement (talus et terrasses) aux phases inférieure et moyenne du Magdalénien, plusieurs travaux individuels ont été réalisés sur différents registres (lithique, parure, ossements). Une réévaluation collective dans un cadre stratigraphique critiqué et chronologique renouvelé s'avérait néanmoins nécessaire.

Afin de préciser le processus de mise en place du Magdalénien dans sa période « classique » (phases moyenne et supérieure), des membres du projet « Magdatis » ont analysé plusieurs gisements attribués au Magdalénien moyen ancien de Gironde. La séquence des fouilles Trécolle s'est naturellement placée au cœur de nos recherches. Dans un premier temps, un retour sur les archives de terrain laissées par ce fouilleur minutieux a été nécessaire. Cette révision archéostratigraphique a conduit à répartir une large part des vestiges en deux ensembles tout en pondérant certaines interfaces problématiques. Partant de cette nouvelle proposition de découpage de la séquence, une réévaluation du matériel archéologique a été entreprise. La comparaison des industries lithiques et osseuses apporte de nouvelles données concernant la nature des comportements techniques et économiques de ces premiers moments du Magdalénien en Europe de l'Ouest. Du côté des matières premières lithiques ou osseuses, on note une certaine continuité des deux ensembles. La gestion différentielle de l'outillage domestique et de l'équipement de chasse en silex s'avère également un levier dialectique efficace pour appréhender la transformation de ces sociétés de chasseurs-cueilleurs. L'industrie osseuse montre, entre les deux ensembles, des éléments de continuité (extraction de baguettes par double rainurage) et des différences (dans l'ensemble supérieur : outillage moins diversifié, objets décorés plus fréquents). La systématisation d'une production laminaire normée dans la seconde phase s'accompagwne d'une augmentation des gabarits des pointes osseuses. La confection de morphotypes différents d'armatures lithiques (microlamelles à dos $v_{s}$ lamelles scalènes) et l'évolution des types de base des pointes osseuses mais aussi la présence ou non de rainures illustrent une recomposition de l'armement. La révision de la faune a également permis de préciser chacun des ensembles et, grâce à l'étude taphonomique, les tableaux de chasse respectifs. Des différences dans les stratégies de chasse des saïgas (groupes de femelles ou hardes mixtes) et leur traitement (intensité de la recherche de moelle, techniques de fracturation) sont notées. La mise en évidence d'une exploitation de gibiers de petite taille (avifaune et mésofaune) dès les premières occupations magdaléniennes vient compléter l'image de ces chasseurs d'antilopes et de rennes. Le croisement des différentes données offre l'opportunité de préciser les processus et les rythmes des changements techniques et économiques qui marquent cette période. De nouvelles datations ${ }^{14} \mathrm{C}$ directes sur espèces ou objets particuliers permettent en outre d'éclairer sous un nouveau jour deux étapes de mise en place du Magdalénien. La séquence des fouilles Trécolle peut ainsi être replacée dans l'ensemble du site de Saint-Germain-la-Rivière et comparée à d'autres gisements contemporains d'Europe de l'Ouest.

Mots clés : Magdalénien, archéostratigraphie, taphonomie, équipement domestique, armement de chasse, technologie lithique, technologie osseuse, saïga, chouette harfang.

Abstract: The Magdalenian site of Saint-Germain-la-Rivière (Gironde, France) has often figured in debates and discussions about the Late Upper Palaeolithic of Western Europe. Most of the discussions are based on the material excavated by R. Blanchard-in particular the now well-known burial - as well as the more rigorous excavations by G. Trécolle in the slope deposits. After the detailed study of all this material by M. Lenoir, which allowed the material from the slope and terrace deposits to be attributed to the Lower and Middle Magdalenian, many other studies were carried out by various researchers on specific aspects of the collections (lithics, personal ornaments, fauna). It became clear, however, that the stratigraphic framework and chronology of the site needed to be reexamined.

In order to understand the rise of the classic Magdalenian in Gironde, members of the Magdatis project reexamined many early Middle Magdalenian sites and collections of that area. The thorough work of Trécolle at Saint-Germain-la-Rivière was pivotal to this reas- 
sessment. Initially, this involved the analysis of his fieldnotes which led to an archaeostratigraphic reattribution of most artefacts into two principal units, with a potentially problematic interface between these. Once these new archaeostratigraphic divisions had been determined, analysis of the artefacts could proceed. The comparison of the lithic and bone/antler industries with those of other sites has afforded us new insights into the nature of the types of technical and economic behaviour of this initial Magdalenian in Western Europe. In terms of lithic and osseous raw material, we have found a good degree of continuity between the two units. The differing treatment of domestic versus hunting flint tools is also an important aspect to consider in the discussion of the changes that took place in huntergatherer societies at this time. The bone/antler industry shows some continuity between units (for example the use of the groove and splinter technique), but also some differences (in the upper unit: less diversity in tools; a higher frequency of decorated objects). The systematization of standardized blade production in the upper unit is accompanied by an increase in size of antler points. The production of different lithic projectile morphotypes (backed microbladelets versus scalene bladelets), in parallel with a change in base types within antler points, in addition to the presence or absence of grooves, demonstrate that changes occurred in the toolkits used. A reassessment of the faunal remains also helped to clarify the practices having occurred in each of the two principal stratigraphic units; combined with a taphonomic analysis, the specific subsistence practices in each could be identified. Different hunting strategies were noticed regarding saiga antelope (hunting within female groups versus mixed herds) as well as differing carcass treatment (degree of intensity of marrow extraction, carcass processing techniques). Our analysis also revealed that small-game hunting (birds and small mammals) had already been practised in the earliest Magdalenian phases at the site, thus providing a more complete picture of these reindeer and saiga antelope hunters. Taking into account all of these data and practices allows us to be more specific about the type and pace of technical and subsistence changes having occurred during this period. New $14 \mathrm{C}$ dates taken directly from bones of identified species or characteristic organic artefacts also aid us in shedding new light on the two main phases of the Magdalenian. The Trécolle excavation and its collections can thus be placed within the overall Saint-Germain-la-Rivière site sequence and compared with other contemporaneous sites in Western Europe which have also benefited from a renewed stratigraphic, archaeological and chronological assessment and framework.

Keywords : Magdalenian, Archaeostratigraphy, Taphonomy, Domestic toolkit, Hunting equipment, Lithic technology, Bone technology, Saiga antelope, Snowy owl.

$\mathrm{D}$ EPUIS une vingtaine d'années, le site magdalénien de Saint-Germain-la-Rivière (SG dans la suite du texte) a régulièrement été sollicité dans le cadre de plusieurs débats concernant le Paléolithique supérieur récent d'Europe de l'Ouest. Qu'il s'agisse de la redéfinition typo-technologique des premières phases du Magdalénien (Lenoir et al., 1994 ; Cazals, 2005 ; Langlais, 2007a et b), des questions d'alimentation et de subsistance à cette période (Costamagno, 1999 et 2001 ; Drucker et al., 2005) ou encore de la caractérisation des pratiques funéraires (Gambier et al., 2000; Henry-Gambier et al., 2002) et de leur interprétation comme de possibles indices d'émergence d'inégalités sociales (Vanhaeren et D'Errico, 2003), SG a souvent été considéré comme un site clé et ses données placées au premier plan de la discussion. L'attention des chercheurs s'est focalisée sur la fameuse sépulture découverte en 1934 par R. Blanchard, mais également sur les résultats des fouilles conduites par G. Trécolle dans les années 1960. En effet, par rapport aux opérations ayant eu lieu sur le site dans les années 1930, les fouilles Trécolle ont bénéficié de techniques de récolte et d'enregistrement de qualité (objets coordonnés en trois dimensions, observations stratigraphiques, tamisage fin). Surtout, les fouilles Trécolle représentent notre unique fenêtre d'observation sur les niveaux les plus anciens du site - niveaux particulièrement importants pour caractériser une phase inférieure du Magdalénien dans le Sud-Ouest français (voir ci-dessous).

Cependant, bien qu'il ait constitué le meilleur échantillon archéologique du site de SG, le matériel issu des fouilles Trécolle posait plusieurs problèmes. Tout d'abord parce qu'il demeurait incomplètement publié : l'industrie osseuse, les mésomammifères ou encore l'avifaune n'avaient pas fait l'objet d'une étude détaillée par un spécialiste, offrant ainsi une vision tronquée des activités pratiquées à SG par les groupes magdaléniens. Par ailleurs, le cadre ${ }^{14} \mathrm{C}$ des fouilles Trécolle restait incomplet, seules deux des cinq couches distinguées par le fouilleur ( $\mathrm{C} 1$ et $\mathrm{C} 4$ ) ayant bénéficié de datations radiocarbone par spectrométrie de masse par accélérateur (AMS). Enfin, la stratigraphie demandait à être rediscutée. En effet, bien que le matériel des fouilles Trécolle ait toujours été décrit en suivant la subdivision en cinq couches établie lors de la fouille (de haut en bas : C, C1, C2, C3, C4), l'étude des industries a montré que ces distinctions, fondées sur des critères sédimentaires, ne correspondaient pas forcément à l'archéostratigraphie ${ }^{(1)}$ (voir ci-dessous). Il fallait donc réviser et compléter ces données.

Revisiter ainsi les séquences archéologiques de référence n'est bien sûr pas une entreprise spécifique à SG. De nombreuses publications récentes en soulignent la nécessité afin de préciser le rythme des transformations culturelles durant la fin du Dernier Maximum Glaciaire (e. g. Bodu et al., 2007; Ducasse et Langlais, 2007; Langlais et al., 2007; Primault et al., 2007; Pétillon et al., 2008; Langlais et al., 2010; Ducasse et al., 2011; Pétillon et Ducasse, 2012; Langlais et Ducasse, 2013). C'est donc dans le but de mieux caractériser les premiers temps du Magdalénien (relations avec son ancêtre badegoulien, rythmes d'évolution des différents équipements et des stratégies d'exploitation des gibiers, processus de mise en place de nouvelles normes...), à travers un des rares gisements documentant en stratigraphie le Magdalénien inférieur et le Magdalénien moyen, que les auteurs de cet article ont mené une réévaluation des données Trécolle en 2012-2014 dans le cadre du projet « Magdatis ». Les résultats de ce travail collectif sont présentés ici, et des comparaisons avec d'autres sites permettent d'ouvrir 
des pistes de réflexion sur les débuts du Magdalénien en Gironde et plus largement dans un grand Sud-Ouest de la France (fig. 1).

\section{HISTORIQUE DES TRAVAUX}

T e site de SG est connu localement sous le nom d' " abri de Pille-Bourse ». Il offre une vue panoramique sur la basse vallée de la Dordogne peu après sa confluence avec l'Isle. Le gisement est constitué de deux abris et replats juxtaposés constituant les terrasses inférieure et supérieure. Ce complexe est prolongé par un talus orienté nord-sud qui plonge en direction de la plaine alluviale. En partie épargné par des carriers friands du calcaire local, ce gisement a fait l'objet de fouilles aux méthodologies variées tout au long $\mathrm{du} \mathrm{XX}^{\mathrm{e}}$ siècle. Après sa découverte en 1929, H. Mirande et R. Lépront mènent des travaux dans le talus et la terrasse supérieure (fig. 2) où ils découvrent notamment un premier squelette humain, tombé en poussière lors de son prélèvement (Mirande et Lépront, 1933; Garde, 1934). La présence de certaines pièces d'industrie osseuse décorées ou non (Lenoir, 2000), de grandes lames et d'armatures en silex sert alors d'élément de définition du Magdalénien II et III.

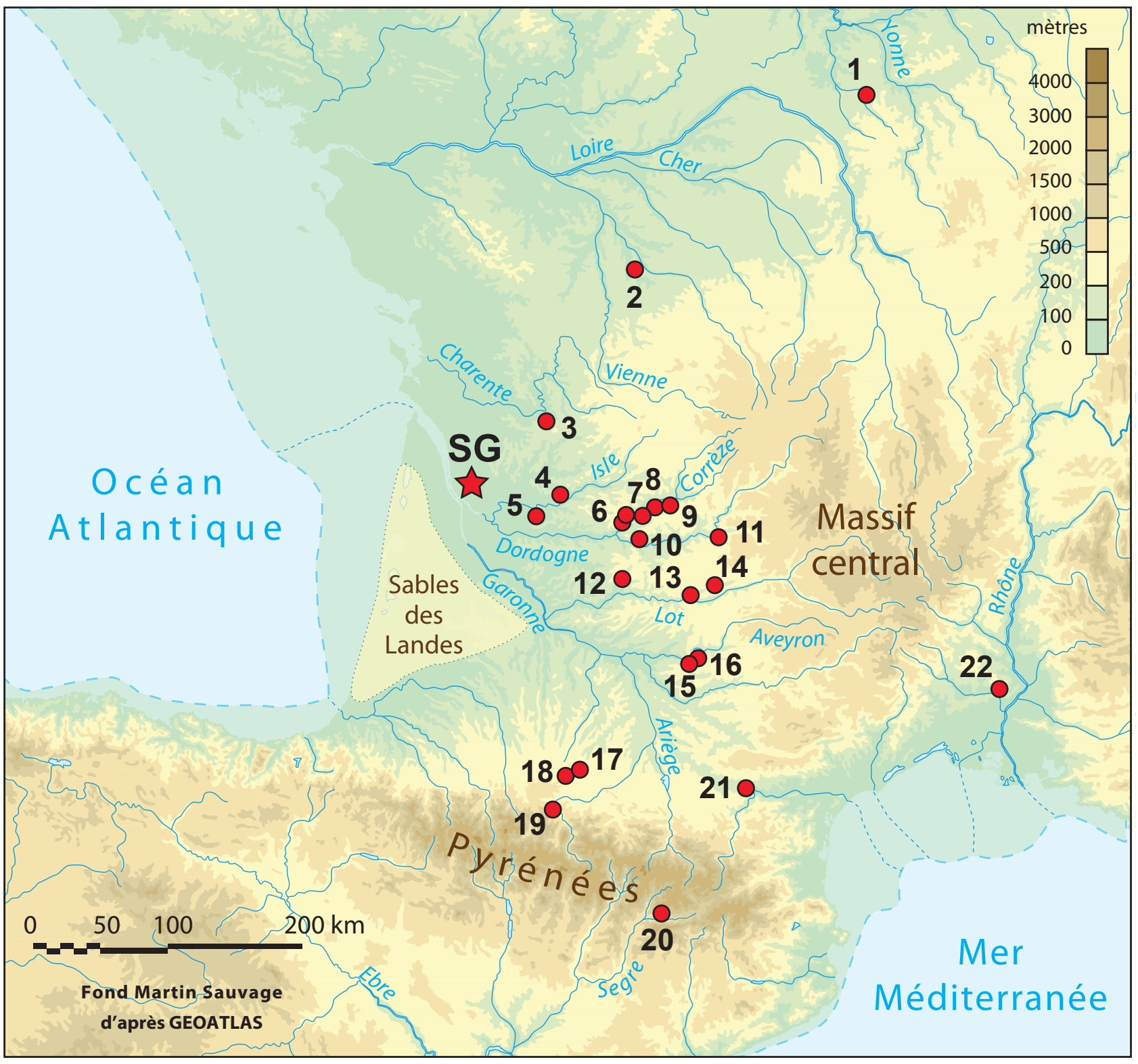

Fig. 1 - Localisation du site de SG et des gisements mentionnés dans le texte (SG : Saint-Germain-la-Rivière, $1:$ Thèmes, $2:$ Taillis des Coteaux, 3 : Chaire-à-Calvin, 4 : Chancelade, 5 : Gabillou, 6 : Laugerie Haute \& Basse, 7 : Cap Blanc, $8:$ Reverdit, $9:$ Lascaux, 10 : Flageolet, 11 : Combe-Cullier, 12 : Le Martinet, 13 : Petit Cloup Barrat, 14 : Peyrugues, $15:$ Lafaye, $16:$ Gandil, $17:$ Les Scilles, 18 : Les Harpons, 19 : Troubat, 20 : Montlleo, 21 : Lassac, 22 : Fontgrasse).

Fig. 1 - Location of $S G$ and other sites mentioned in the text (see list above). 


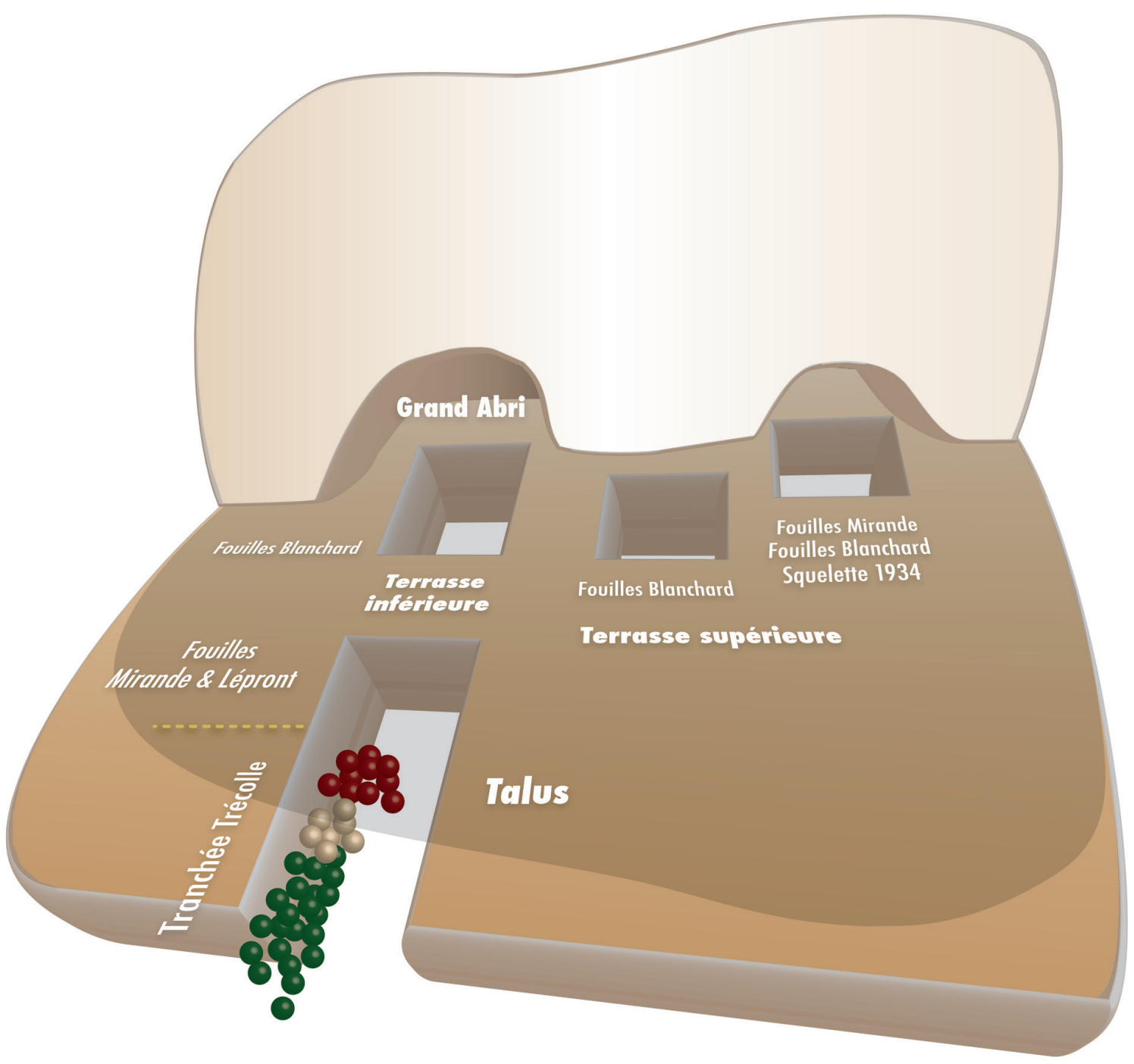

Fig. 2 - SG : plan schématique du gisement et localisation des interventions archéologiques. Points rouges : ensemble supérieur; points gris : ensemble médian; points verts : ensemble inférieur (DAO FLC).

Fig. 2 - SG: schematic drawing of the site, indicating the placement of archaeological excavations. Red dots: upper stratigraphic unit; grey dots: middle stratigraphic unit; green dots: lower stratigraphic unit (CAD FLC).

R. Blanchard prend la suite des opérations dans les années 1930 et commence à fouiller le grand abri de la terrasse inférieure (fig. 2). Il y distingue deux couches recouvertes par un éboulis qui masquait des gravures pariétales figuratives (Bouvier et Trécolle, 1966; Blanchard et al., 1972; Dubourg, 1997). La faune est dominée par le couple saïga-renne avec la présence de cheval (Prat, 1968; Blanchard et al., 1972). Certains auteurs attribuent l'industrie lithique et osseuse de la couche inférieure A au Magdalénien II, notamment sur la présence de " triangles » (Peyrony, 1936 et 1938; SonnevilleBordes, 1960), et d'autres au « Saint-Germien » ou "Proto-Magdalénien IIb » (Cheynier et Bouyssonie, 1955; Coulonges, 1956; Cheynier, 1965). D. Peyrony attribue le matériel de la couche B et celui de la terrasse supérieure au Magdalénien III (Blanchard et al.,
1972), ce que confirme D. Sonneville-Bordes (1960). J. Allain propose quant à lui d'intégrer cet ensemble au « Magdalénien à navettes » (Allain et al., 1985). Entre les deux terrasses, R. Blanchard découvre un « amoncellement d'os non brisés surtout de membres d'équidés, de bovidés et de cerfs » qu'il interprète comme une réserve de viande (Blanchard et al., 1972, p. 42). En 1996, M. Lenoir opère une réévaluation de ce secteur. Malgré une date ${ }^{14} \mathrm{C}$ cohérente avec les autres (voir cidessous), ce « magma d'os » pourrait selon lui résulter de mélanges avec des remplissages naturels de cavités voisines, mélanges causés par des travaux de carriers à proximité (Lenoir, 1996). Dans la terrasse supérieure, R. Blanchard met au jour plusieurs vestiges lithiques (notamment de grandes lames semblables à celles de la série Mirande), de l'industrie osseuse et de la faune. Sur- 
tout, il y découvre en 1934 une sépulture humaine bien conservée (Blanchard, 1935; Vaufrey, 1935).

Entre 1959 et 1969, dans le talus qui prolonge la terrasse inférieure, le docteur G. Trécolle réalise une fouille minutieuse sous la forme d'une tranchée de $9 \mathrm{~m}^{2}$ en partant de l'extrémité nord de celle réalisée par H. Mirande une trentaine d'années plus tôt (fig. 2). Il y distingue plusieurs couches archéologiques plus ou moins riches en vestiges lithiques et osseux. L'analyse paléontologique de la faune a fait l'objet d'une thèse à l'université Bordeaux 1 (Ouzrit, 1986). Bien qu'orienté surtout vers les Mammifères, ce travail indique la présence de nombreux restes de chouette harfang. L'étude du matériel lithique permet de distinguer deux grands complexes : un Magdalénien dit « ancien original » sur lequel repose un ensemble attribué au Magdalénien moyen (Lenoir, 1983; Lenoir et al., 1991 et 1995). Une reprise de coupe effectuée dans cette tranchée a bien montré une meilleure conservation des niveaux profonds par rapport à un ensemble supérieur ayant subi les effets gravitaires du talus (Lenoir, 1996).

Comme dit précédemment, les résultats présentés ici portent sur le matériel mis au jour par G. Trécolle. Pour cela, il s'agissait dans un premier temps de redéfinir cette séquence afin de confronter dans un même cadre archéostratigraphique les différents registres d'activité mis en œuvre au cours des premiers temps du Magdalénien.

\section{PROPOSITION D'UN NOUVEAU CADRE ARCHÉOSTRATIGRAPHIQUE POUR LA TRANCHÉE TRÉCOLLE}

$\mathrm{L}$ es fouilles Trécolle ont été exemplaires pour l'époque, tant par les méthodes de terrain mises en œuvre que par la tenue d'archives de terrain facilitant largement la réévaluation entreprise ici. L'informatisation des données spatiales issues des carnets de fouilles a été couplée à leur numérisation dans un but d'archivage et de conservation. Comme souvent dans ce type d'exercice, le dépouillement et l'extraction de données réutilisables dans le cadre de nos propres problématiques se sont heurtés aux difficultés d'interprétation des sources issues de fouilles anciennes. Outre les erreurs de notation, graphies illisibles, doublons, équivocité du ou des systèmes de carroyage, l'attribution stratigraphique des objets, telle qu'enregistrée à l'époque, est particulièrement délicate. En effet, différents niveaux d'incertitude se superposent : absence de notation pour de longues séries; contradiction entre notation sur archive et marquage de la pièce; valeurs des repères verticaux de référence absents, incohérents ou contradictoires; ambiguïté du caractère absolu ou relatif des profondeurs notées par rapport au zéro. De plus, l'hétérogénéité et la multiplicité des modalités ou appellations stratigraphiques transparaissent à travers l'emploi d'environ quatre-vingts notations différentes dans les carnets de fouille (ex : « couche rouge », « foyer $5 »$, « couche 3 rouge »...). Les prises de décision de l'époque, devenues floues car incomplètement archi- vées par écrit, devaient néanmoins être appréciées, et des choix en termes de retranscription devaient être faits. Avant même de réinterpréter l'archéostratigraphie, c'était donc l'enregistrement de sa compréhension de l'époque qui devait être examiné. Comme tout travail de retranscription, cette démarche introduit des distorsions et des erreurs. Mais ce risque, mesuré et assumé, était nécessaire à la poursuite de notre réévaluation. Au terme de ce travail préparatoire, neuf modalités stratigraphiques ont été retenues sous la forme de couches $(\mathrm{C}$ à $\mathrm{C} 4)$ et d'interfaces $(\mathrm{C} / \mathrm{C} 1$ à $\mathrm{C} 3 / \mathrm{C} 4)$. En analysant les points projetés sur plans verticaux, nous avons pu inclure un nombre important d'objets coordonnés dépourvus de toute affectation. Nous avons également pu contrôler la répartition stratigraphique des neuf modalités dans chaque mètre carré (fig. 3). Cette réattribution a permis d'injecter au sein des tableaux de décompte les éléments issus des refus de tamis (marqués uniquement par couche et carré) étudiés par chacun des membres de l'équipe. On remarque ainsi que les modalités supérieures sont essentiellement documentées dans la partie haute du gisement (bandes 20-21). Nous avons ensuite projeté sur un plan sagittal des marqueurs lithiques et osseux coordonnés, définis selon des critères techno-typologiques et par comparaisons avec d'autres gisements (voir ci-dessous). Comme la succession des modalités, la répartition des marqueurs confirme l'existence de deux ensembles différents dans la partie amont du talus, fouillée en premier (fig. 4). La stratigraphie complète telle que définie par Trécolle ne s'applique donc pas à l'ensemble de sa tranchée. En travaillant sur chaque carré, nous avons pu réattribuer l'essentiel du matériel et proposer une nouvelle archéostratigraphie (fig. 5 et 6$)^{(2)}$. Ce premier travail aboutit à distinguer deux ensembles, inférieur et supérieur, séparés par un ensemble médian. Ce dernier, constitué par des pièces difficiles à réattribuer et l'association de marqueurs des ensembles sous et sus-jacents, a été exclu de l'analyse en tant qu'interface mélangée. Cette proposition de découpage archéostratigraphique constitue le cadre unique de l'étude des différents registres présentés ici, qu'il s'agisse des équipements lithique et osseux ou de la faune.

\section{LES ÉQUIPEMENTS EN SILEX}

$\mathrm{L}$ a séquence Trécolle permet de comparer les stratégies mises en œuvre au cours des premières phases du Magdalénien dans un même environnement lithologique pour la confection des panoplies en silex. Du côté des matières premières, on note une homogénéité des deux ensembles avec une nette préférence $(>80 \%)$ pour des silex de bonne qualité disponibles localement dans les terrasses alluviales de la Dordogne (Sénoniens grisnoirs ou blonds et Maastrichtiens) et les plateaux voisins (Tertiaires). Parmi les matériaux allochtones, le silex « grain de mil » a été déterminé $(<5 \%)$ dans les deux ensembles (Langlais, 2007a). Disponible en Charente à une cinquantaine de kilomètres au nord du gisement 

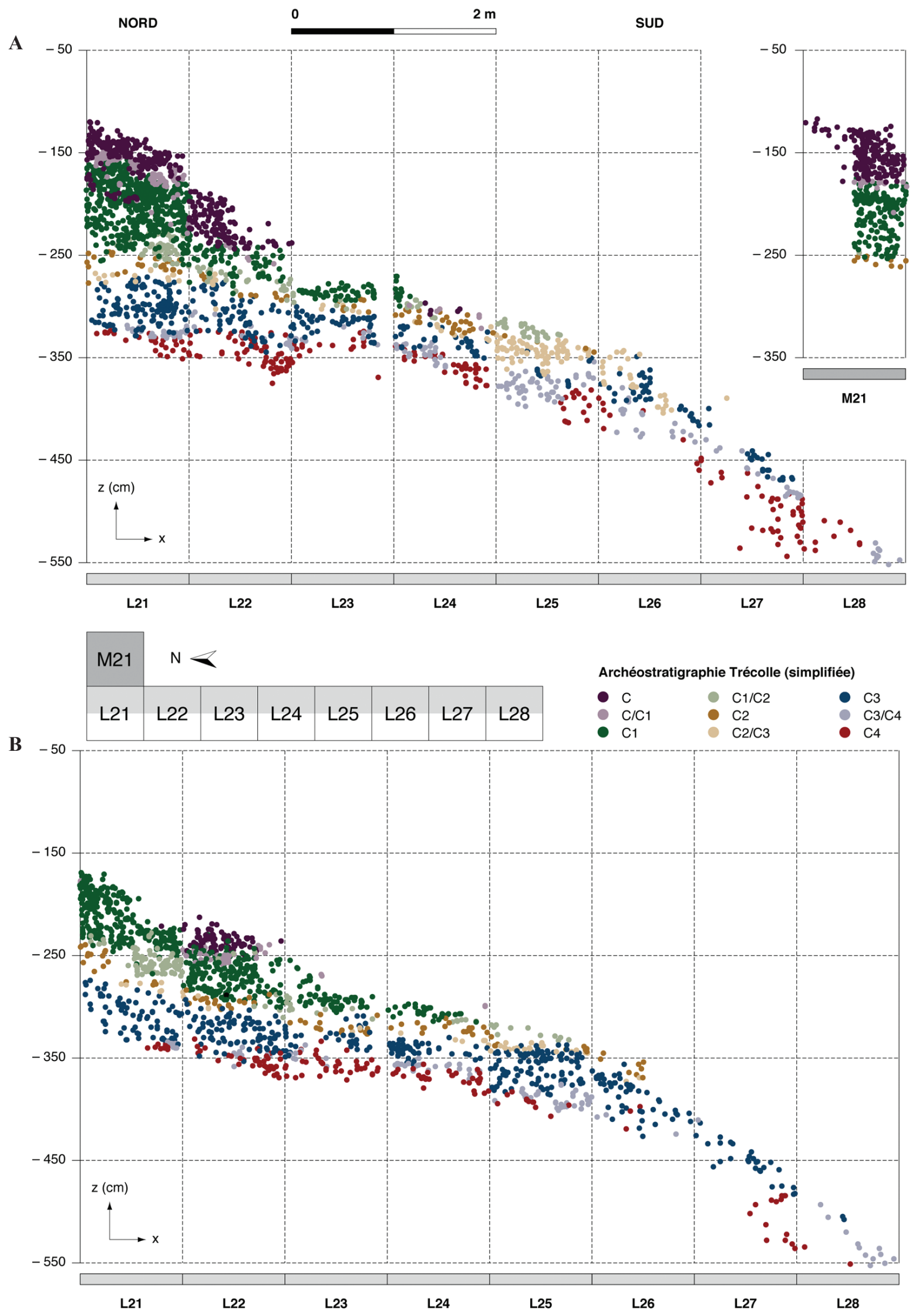

Fig. 3 - SG : projection sur plan vertical des vestiges coordonnés selon les modalités stratigraphiques de terrain simplifiées. A : travée $50-100 \mathrm{~cm}$ et carré M21; B : travée 0-50 cm (DAO FLC).

Fig. 3 - SG: Scatterplot of the artefacts recorded at $S G$ according to the simplified stratigraphic data determined during fieldwork. A: transect 50-100 cm and square M21;B: transect 0-50 cm (CAD FLC). 


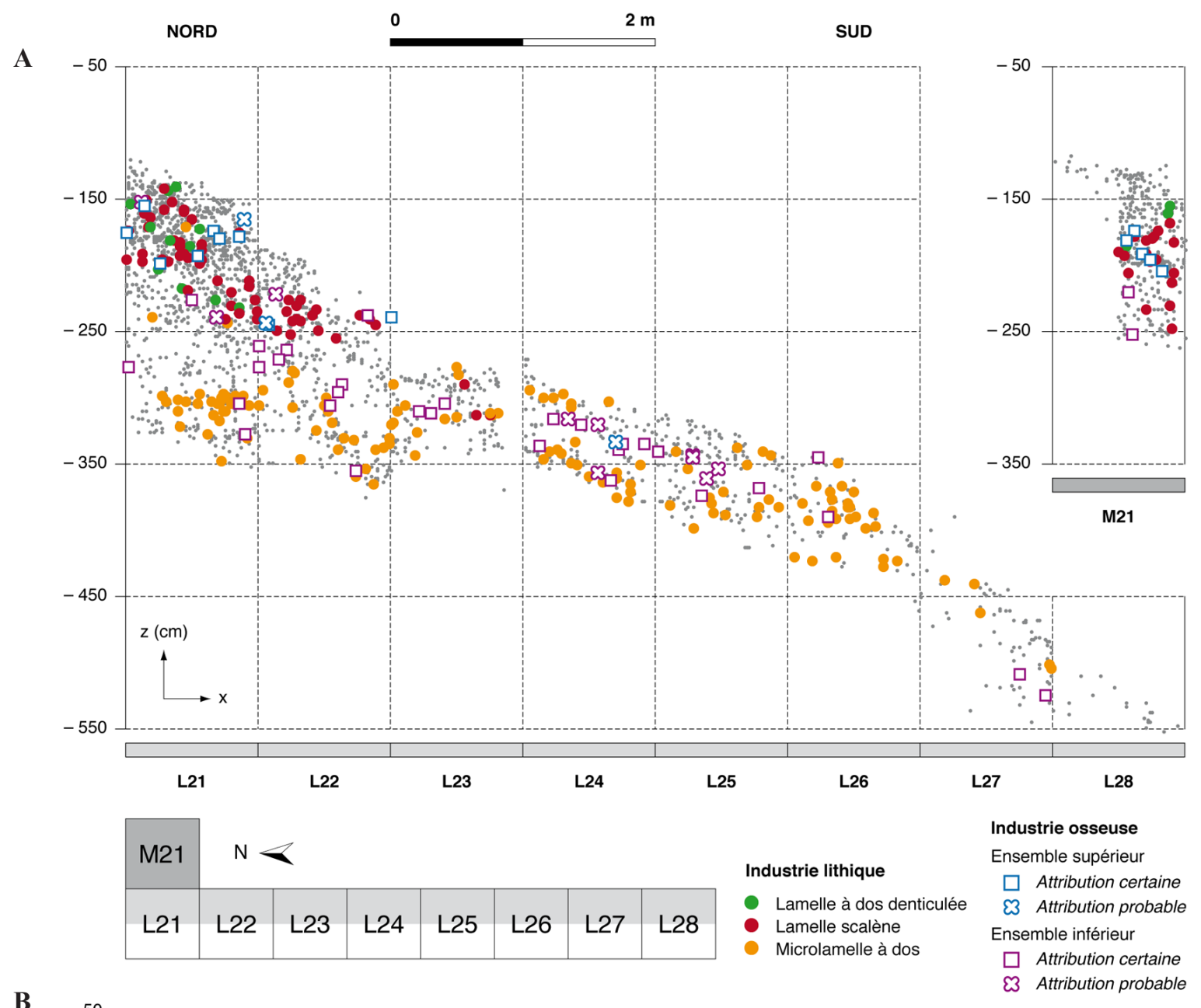

B

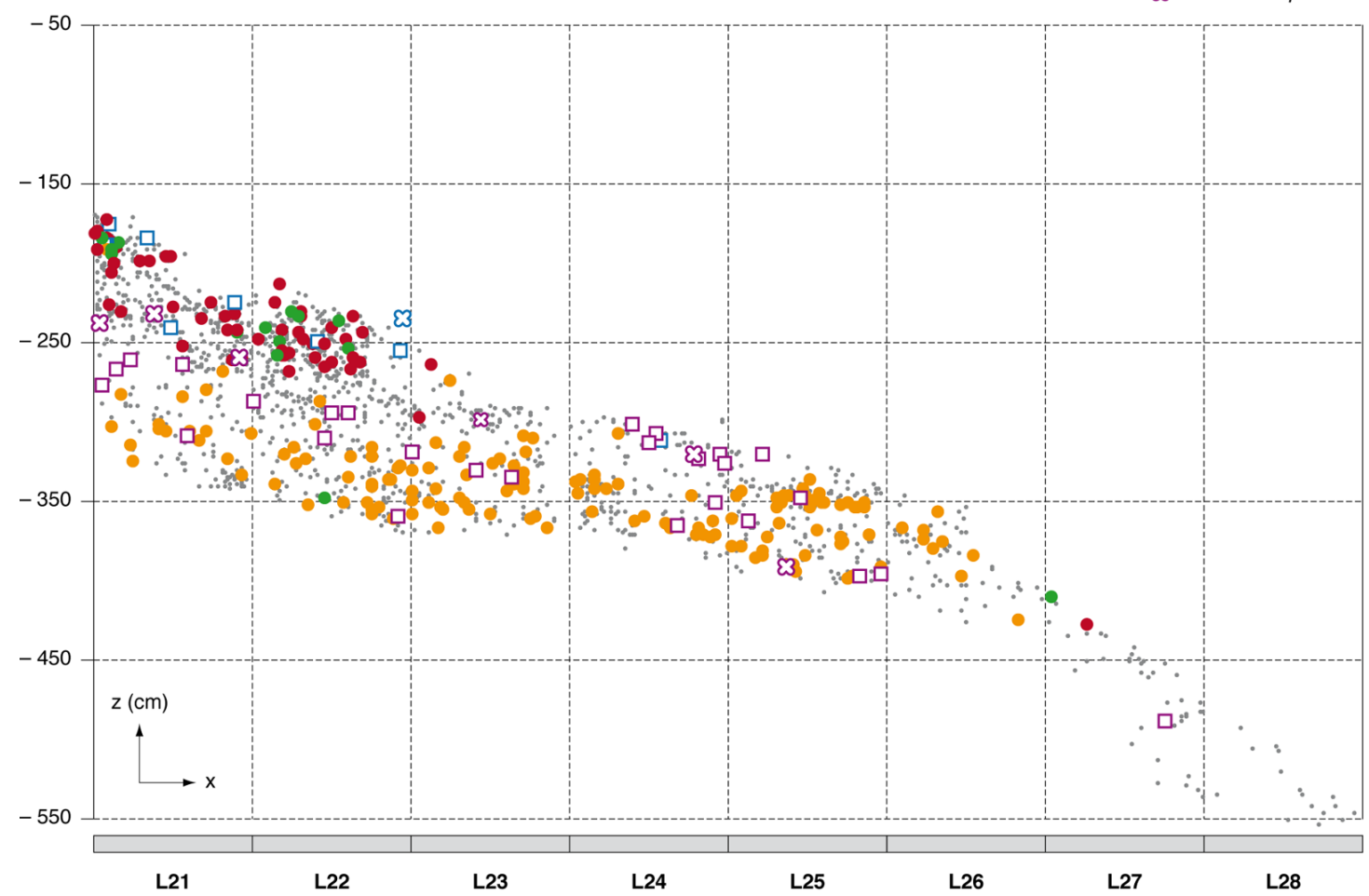

Fig. 4 - SG : projection sur plan vertical des marqueurs lithiques et osseux coordonnés. A : travée 50-100 cm et carré M21; B : travée 0-50 cm (DAO FLC).

Fig. 4 - SG: Scatterplot of lithic and osseous culturally diagnostic artefacts. A: transect 50-100 cm and square M21;B: transect $0-50 \mathrm{~cm}$ (CAD FLC). 

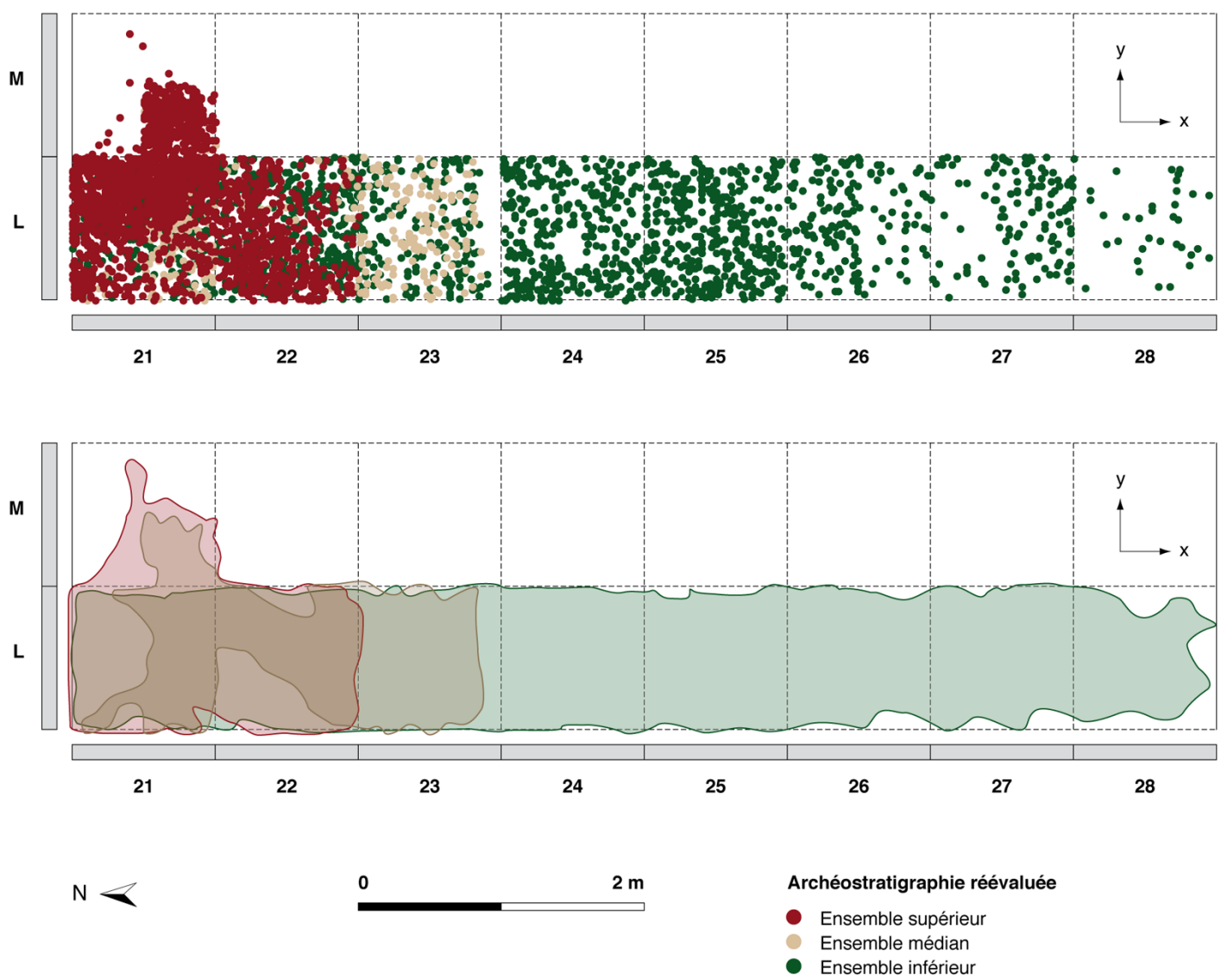

Fig. 5 - SG : projection sur plan horizontal des vestiges coordonnés selon la nouvelle archéostratigraphie (DAO FLC).

Fig. 5 - SG : scatterplot of artefacts based on the new archaeostratigraphy (CAD FLC).

(Simonnet et al., 2007; Caux, 2014), il est importé sous la forme de supports et vraisemblablement de rognons dans l'ensemble supérieur comme en témoignent quelques déchets de taille. L'ensemble supérieur livre une plus grande diversité de silex allochtones qui nécessiterait une étude pétroarchéologique poussée. Néanmoins, on peut en l'état souligner l'absence de matériaux d'origine lointaine (Touraine, Poitou, Chalosse, Lot-et-Garonne) bien documentés dans les autres séries de SG (voir ci-dessous).

Bien que d'extension spatiale différente, les deux ensembles ont livré chacun une dizaine de milliers de restes en silex environ, toutes fractions confondues. Dans l'ensemble inférieur, mises à part les esquilles infracentimétriques, la série $(\mathrm{n}=6220)$ est composée pour environ $40 \%$ de supports lamellaires et pour près de $10 \%$ d'éclats laminaires et de lames. L'autre moitié de la série correspond pour une large part à des déchets de ces chaînes opératoires et pour une autre part à des supports provenant de débitages d'éclats autonomes. Dans l'ensemble supérieur, une fois exclus les vestiges infracentimétriques, on obtient un total de 4090 pièces. Les produits lamellaires représentent également $40 \%$ mais les lames et éclats laminaires comptent désormais pour $25 \%$ de l'ensemble. Le reste provient de la mise en œuvre de ces productions lamino-lamellaires. Bien que déjà considérées à l'époque comme des nucléus à lamelles (Lenoir et al., 1991), plu- sieurs pièces comme les burins et grattoirs de type caréné apparaissaient dans les décomptes typologiques (Lenoir, 1983; Lenoir et al., 1994). Nous les avons donc exclues du tableau de l'outillage, comme certaines pièces dites « à encoches ou denticulées » parmi lesquelles se cachent plusieurs ébauches de nucléus carénés. La description de ces industries ayant déjà fait l'objet de publications récentes (Lenoir 2000; Langlais, 2010), nous nous attachons ici à en présenter les grandes tendances au sein des deux ensembles.

\section{L'équipement domestique}

L'outillage en silex de l'ensemble inférieur est préférentiellement réalisé sur des éclats laminaires plus ou moins épais et des lames (fig. 7 et 8) mais aussi sur quelques éclats courts (fig. 9). Les burins dominent, suivis des grattoirs, des pièces tronquées, retouchées ou esquillées (tabl. 1). Nous avons exclu du décompte des fragments à retouche partielle irrégulière - parmi lesquels il est parfois délicat de distinguer ce qui relève d'une retouche taphonomique de ce qui est anthropique (même d'utilisation) - et des «pièces à encoches et denticulées 》 correspondant en fait pour la plupart à des ébauches de nucléus à lamelles. Si quelques lames présentent des stigmates compatibles avec l'emploi d'un percuteur 


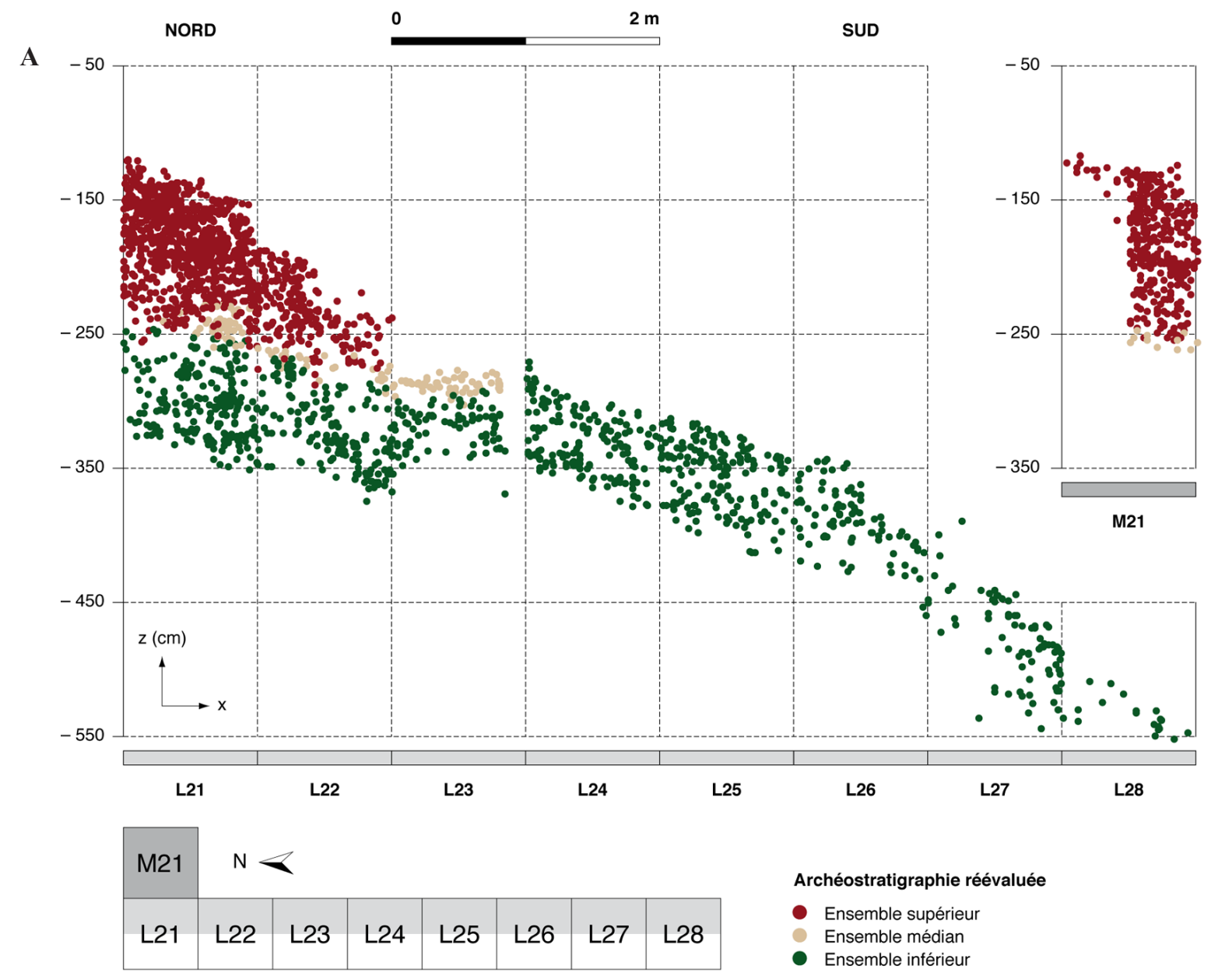

B

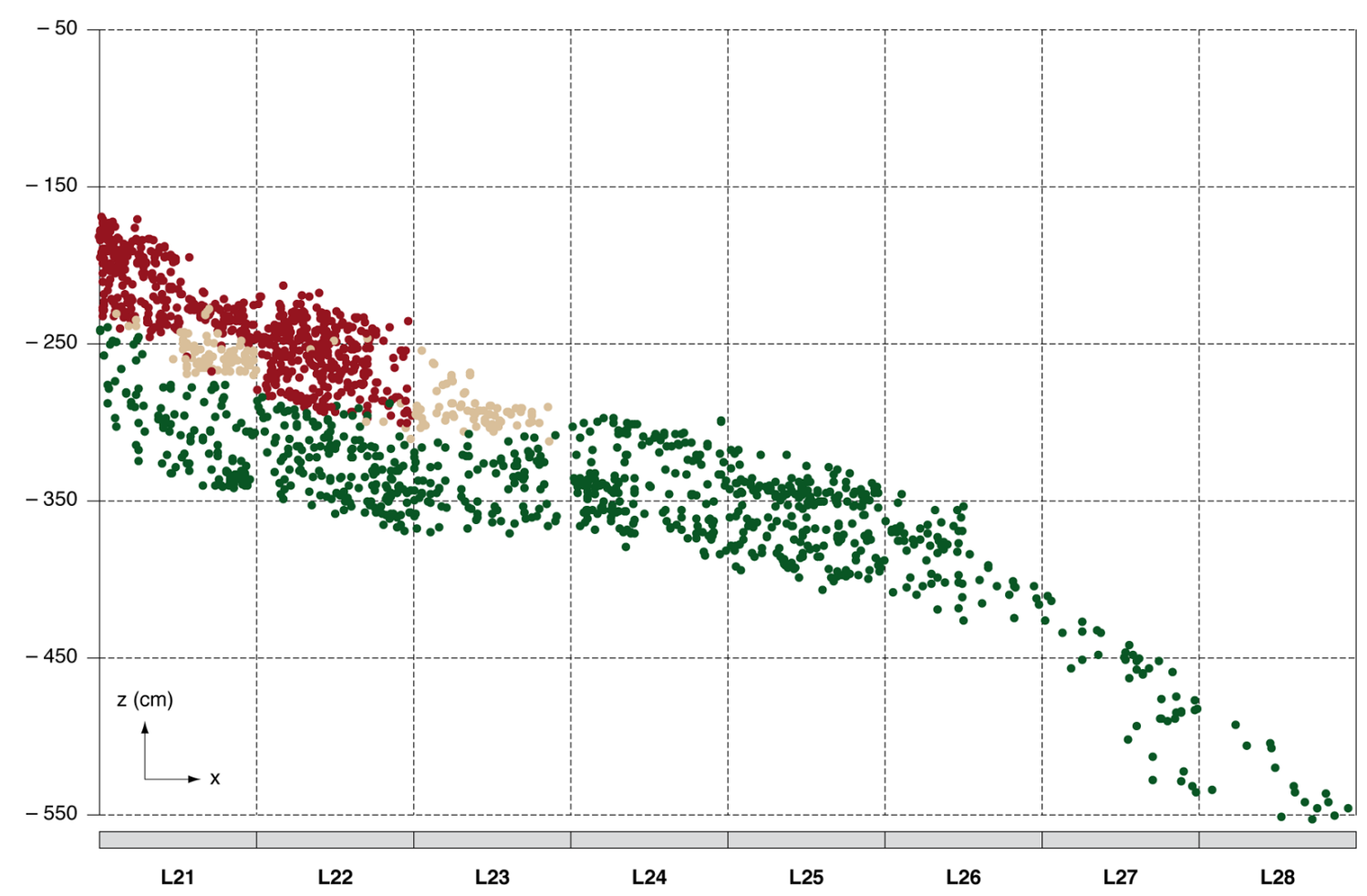

Fig. 6 - SG : projection sur plan vertical des vestiges coordonnés selon la nouvelle archéostratigraphie. A : travée 50-100 cm et carré M21; B : travée 0-50 cm (DAO FLC).

Fig. $6-S G$ : Scatterplot of artefacts based on the new archaeostratigraphy. A: transect 50-100 cm and square M21;B: transect $0-50 \mathrm{~cm}(C A D F L C)$. 

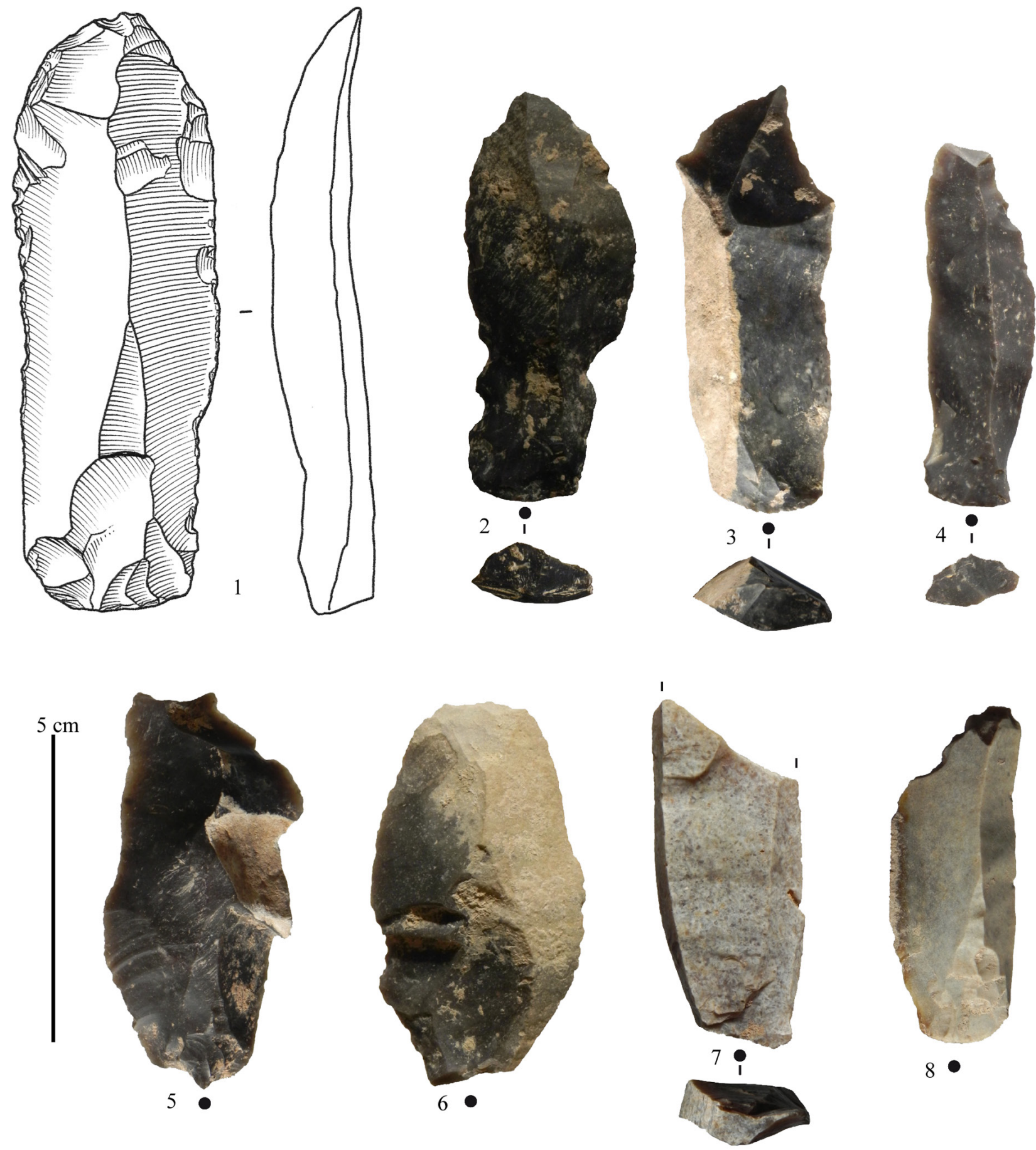

Fig. 7 - SG (ensemble inférieur) : exemples d'outils sur éclats laminaires . 1 : appointé; 2-4, 7 : pièces à retouches marginales partielles; 5-6, 8 : tronqués (dessins S. Pasty, DAO ML).

Fig. 7 -SG (lower stratigraphic unit): examples of tools on laminar flakes. 1: pointed; 2-4, 7: with marginal incomplete retouch; 5-6, 8: truncated (drawings S. Pasty, CAD ML).

tendre organique, la majorité des supports de première intention provient d'un débitage soigné à la pierre. Le schéma unipolairese se caractérise par une faible mise en forme (ouverture corticale ou plus rarement avec crête d'entame) et un entretien limité des convexités (quelques néocrêtes). Des lames et des éclats laminaires sont transformés en outils. Les premières présentent un talon soigneusement abrasé tandis que les seconds sont généra- lement plus épais du fait d'une percussion en retrait de la corniche. Dans l'équipement abandonné sur place, on remarque ainsi une faible standardisation des outils. Ceci est accentué par la présence de quelques éclats courts et minces (fig. 9, nos 1-3) issus vraisemblablement d'une chaîne opératoire autonome, bien que marginale dans cet assemblage (Langlais, 2010). La diversité morphométrique des supports d'outils de l'ensemble inférieur de SG 

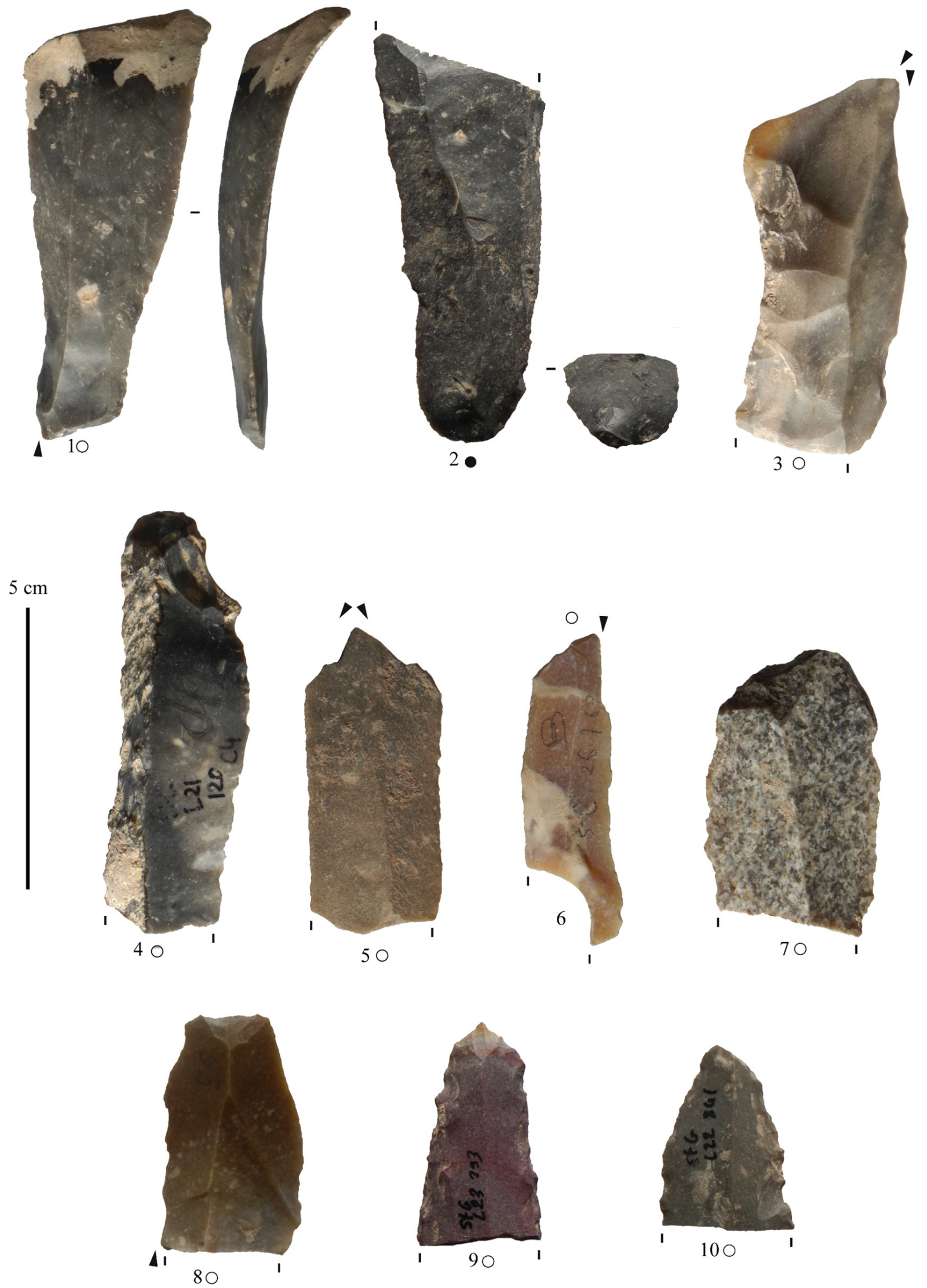

Fig. 8 - SG (ensemble inférieur) : exemples d'outils sur lames. 1, 3, 5-6: burins; 2 : lame à retouche marginale; 4, $7:$ grattoirs; 8 : burin sur troncature; 9-10 : lames retouchées appointées (DAO ML).

Fig. 8-SG (lower stratigraphic unit) : examples of tools on blades. 1, 3, 5-6: burins; 2: blade with marginal retouch; 4, 7: endscrapers; 8: burin/truncation; 9-10: pointed and retouched blades (CAD ML). 


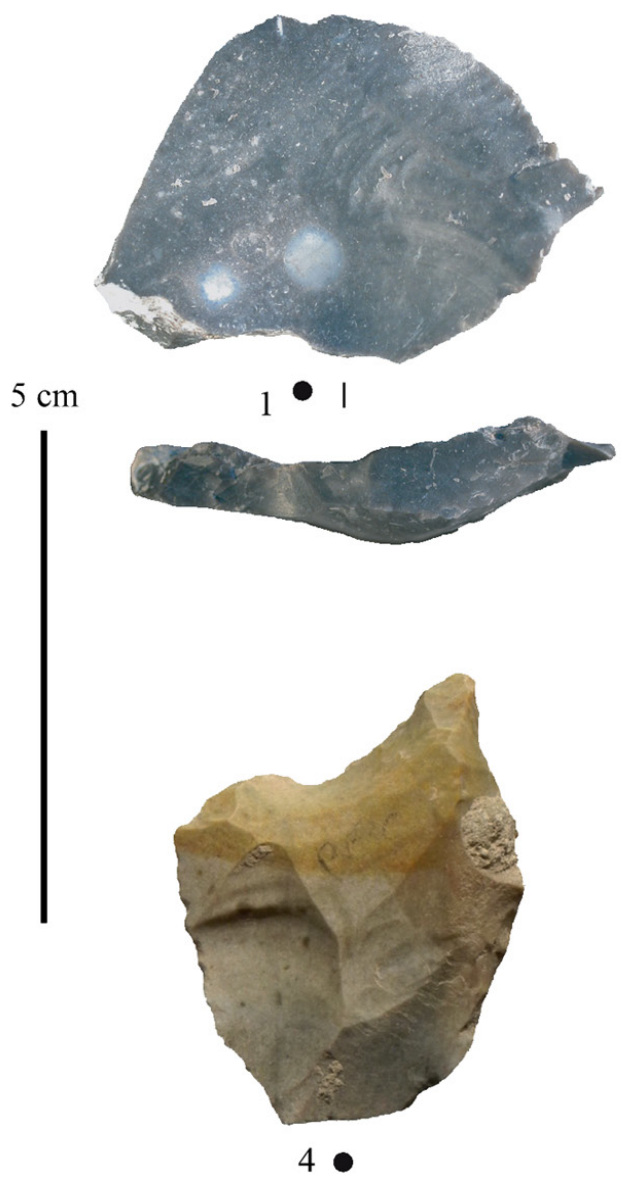

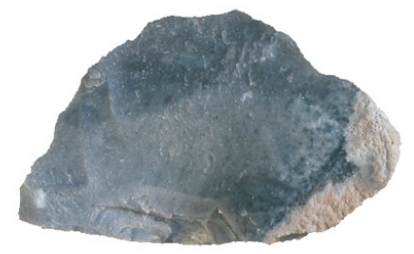

2
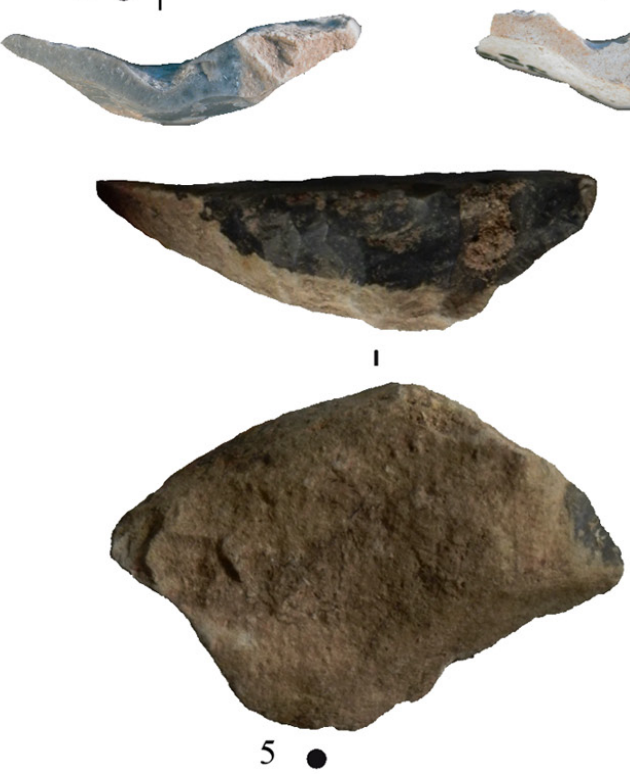

Fig. 9 - SG (ensemble inférieur) : exemples d'outils sur éclats. 1-3 : éclats minces à retouche marginale partielle; $4:$ bec; 5 : grattoir (DAO ML).

Fig. 9 -SG (lower stratigraphic unit) : examples of tools on flakes. 1-3: thin flakes with marginal incomplete retouch; 4: bec; 5: endscraper (CAD ML).

\begin{tabular}{|c|c|c|c|c|c|}
\hline \multirow{2}{*}{\multicolumn{2}{|c|}{ Types }} & \multicolumn{2}{|c|}{ Inférieur } & \multicolumn{2}{|c|}{ Supérieur } \\
\hline & & NR & \multirow{2}{*}{$\%$} & NR & \multirow{2}{*}{$\%$} \\
\hline \multirow{3}{*}{ Burin } & dièdre & 33 & & \multirow{3}{*}{$\begin{array}{l}32 \\
26\end{array}$} & \\
\hline & sur troncature & 2375 & \multirow[t]{2}{*}{36,9} & & \multirow[t]{2}{*}{28,0} \\
\hline & sur cassure & 19 & & & \\
\hline \multirow{3}{*}{ Grattoir } & simple & 44 & \multirow{3}{*}{22,7} & \multirow{3}{*}{93} & \multirow{3}{*}{29,2} \\
\hline & double & -46 & & & \\
\hline & sur lame ret. & 2 & & & \\
\hline \multicolumn{2}{|c|}{$\begin{array}{l}\text { Lame/éclat laminaire } \\
\text { tronqué }\end{array}$} & 24 & 11,8 & 31 & 9,7 \\
\hline \multicolumn{2}{|c|}{$\begin{array}{l}\text { Lame/éclat laminaire } \\
\text { retouché }\end{array}$} & 21 & 10,3 & 71 & 22,3 \\
\hline \multicolumn{2}{|c|}{ Pièce esquillée } & 17 & 8,4 & 3 & 0,9 \\
\hline \multicolumn{2}{|c|}{ Éclat retouché } & 13 & 6,4 & - & - \\
\hline \multicolumn{2}{|c|}{$\begin{array}{l}\text { Lame retouchée } \\
\text { appointée }\end{array}$} & 2 & 1,0 & 22 & 6,9 \\
\hline \multicolumn{2}{|c|}{ Grattoir-burin } & 3 & 1,5 & 7 & 2,2 \\
\hline \multicolumn{2}{|c|}{ Burin-bec } & 1 & 0,5 & - & - \\
\hline \multicolumn{2}{|l|}{$\mathrm{Bec}$} & 1 & 0,5 & 1 & 0,3 \\
\hline \multicolumn{2}{|c|}{ Grattoir-bec } & - & - & 1 & 0,3 \\
\hline \multicolumn{2}{|c|}{ Total équipement } & 203 & 100 & 318 & 100 \\
\hline
\end{tabular}

ne reflète pas un manque de soin mais relève plutôt d'une variété des procédés techniques permettant de se fournir en supports courts ou allongés, minces ou trapus.

Les supports d'outils domestiques de l'ensemble supérieur se différencient de l'ensemble sous-jacent. Il s'agit préférentiellement de lames présentant des gabarits calibrés autour de 15-25 $\mathrm{mm}$ de largeur et 5-8 $\mathrm{mm}$ d'épaisseur. Les outils d'extrémité sont dominés par les grattoirs et burins suivis de lames tronquées ou appointées en ogive (tabl. 1, fig. 10). Sur ces pièces comme sur les outils latéraux, la retouche des tranchants est fréquemment profonde, écailleuse à scalariforme, modifiant fortement les largeurs initiales. L'examen des talons montre cette fois l'emploi préférentiel du percuteur tendre organique. Le plan de frappe est souvent lisse et plus rarement préparé à l'aide d'un facettage localisé. Ainsi, on note la présence de quelques lames munies d'un talon en éperon mais, généralement, une soigneuse abrasion des

Tabl. 1 - SG (Trécolle) : composition typologique de l'outillage en silex par ensemble.

Table 1 -SG (Trécolle): typological list of flint artefacts, organized by unit. 

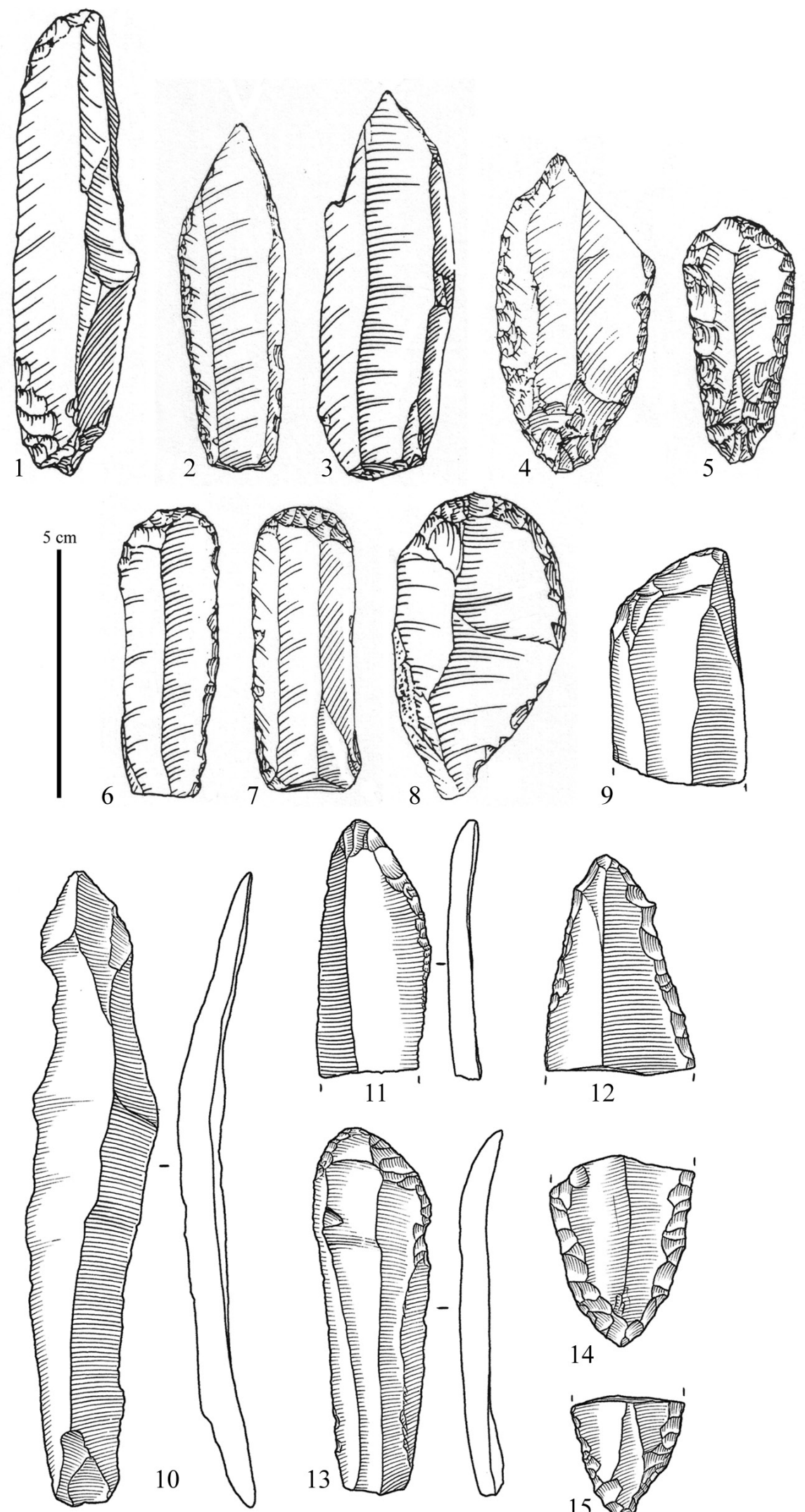

14

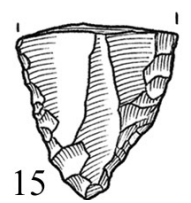

Fig. 10 - SG (ensemble supérieur) : exemples d'outils sur lames. 1-2, $4:$ burin sur lame retouchée/appointée; $3:$ burin; 5-6 : grattoir sur lame retouchée; 7-8: grattoir; 9, 11, 13 : lame appointée ogivale; $10:$ couteau; 12, 14-15: lame appointée (1-8: d'après Lenoir et al., 1994 ;9-15: dessins S. Pasty).

Fig. 10 - SG (upper stratigraphic unit) : examples of tools on blades. 1-2, 4: burins on retouched/pointed blades; 3: burin; 5-6: endscraper on retouched blade; 7-8: endscraper; 9, 11, 13: pointed ogival blades; 10: knive; 12, 14-15: pointed blade (1-8: after Lenoir et al. 1994; 9-15: drawings S. Pasty). 
corniches a suffi. Contrairement à l'ensemble précédent, le débitage laminaire est optimisé en terme de longueur disponible sur les volumes initiaux, de productivité et de normalisation des supports. Des aménagements trans- versaux permettent de gérer les convexités du volume nécessaires à une bonne régularité du débitage unipolaire (fig. 11). À moins de considérer que la représentativité de la fenêtre de fouilles ait biaisé l'échantillon analysé,
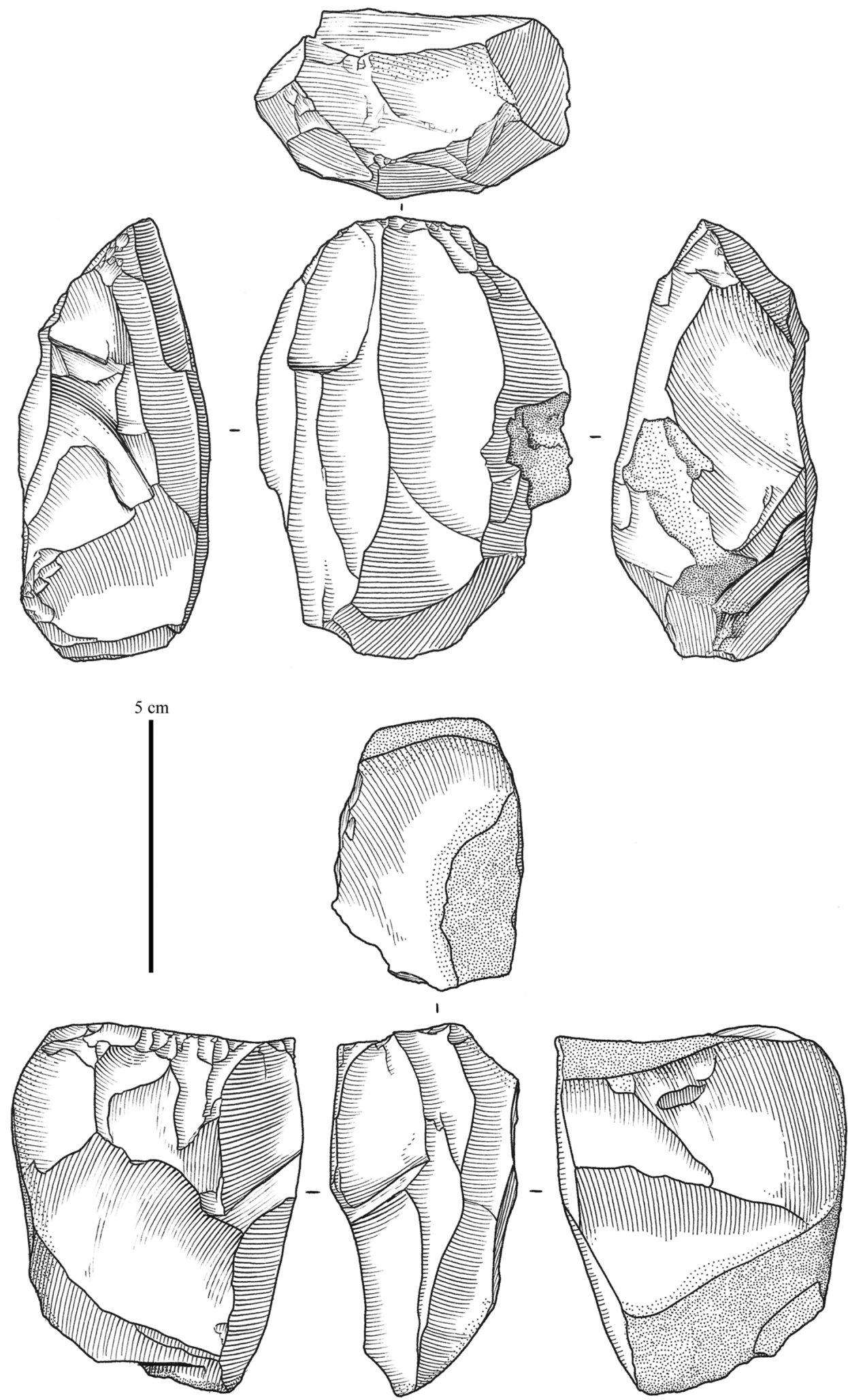

Fig 11 - SG (ensemble supérieur) : exemples de nucléus à lames en phase finale (dessins S. Pasty).

Fig 11 - SG (upper stratigraphic unit) : examples of blade cores in their final exploitation phase (drawings S. Pasty). 


\begin{tabular}{|l|c|c|c|c|}
\multirow{2}{*}{ Types } & \multicolumn{2}{|c|}{ Inférieur } & \multicolumn{2}{c|}{ Supérieur } \\
\cline { 2 - 5 } & NR & $\%$ & NR & $\%$ \\
\hline MicroLD (dont appointée) & $\mathbf{9 3 4}$ & $\mathbf{9 1 , 3 9}$ & 5 & 0,86 \\
\hline LD simple & 71 & 6,95 & $\mathbf{2 7 7}$ & $\mathbf{4 7 , 4 3}$ \\
\hline Lamelle scalène & 4 & 0,39 & $\mathbf{1 8 8}$ & $\mathbf{3 2 , 1 9}$ \\
\hline LD appointée & 10 & 0,98 & 24 & 4,11 \\
\hline LD denticulée & 1 & 0,10 & 33 & 5,65 \\
\hline LD tronquée & 1 & 0,10 & 28 & 4,79 \\
\hline LD étroite & 1 & 0,10 & 19 & 3,25 \\
\hline Lamelle scalène denticulée & - & - & 10 & 1,71 \\
\hline Total armatures & $\mathbf{1 0 2 2}$ & 100 & $\mathbf{5 8 4}$ & 100 \\
\hline
\end{tabular}

Tabl. 2 - SG (Trécolle) : composition typologique des armatures en silex par ensemble (LD : lamelle à dos).

Table 2 - SG (Trécolle): typological list of flint projectiles, organized by unit (LD: backed bladelet).

la rareté des nucléus à lames épuisés s'explique par une réorientation ou un fractionnement des volumes pour des débitages lamellaires (voir ci-dessous). L'absence de supports de dimensions intermédiaires va plutôt dans le sens d'une disjonction opératoire entre les débitages laminaires et lamellaires. Cette dichotomie se reflète également dans les intentions économiques entre des outils sur lames et des armatures sur lamelles.

\section{Les armatures lithiques}

L'ensemble inférieur livre une abondante production (micro)lamellaire destinée à la confection des armatures, comme en témoignent des stigmates diagnostiques d'impact. La diversité des schémas microlamellaires répond à un même besoin en supports de profil à tendance courbe, présentant des gabarits constants. Certains supports montrent une torsion proximale plus ou moins marquée, héritage d'un décalage du point d'impact lors d'un débitage sur une table carénée. Le morphotype largement dominant - utilisé comme marqueur archéostratigraphique - est la microlamelle à dos (tabl. 2, fig. 12). Ce dos, latéralisé à droite dans $75 \%$ des cas (lamelle orientée talon en bas et vue en face supérieure), est généralement direct envahissant, plus rarement direct marginal voire inverse (fig. $12, \mathrm{n}^{\circ} 8$ ). Bien que naturellement pointue, l'extrémité distale - voire parfois l'extrémité proximale est fréquemment appointée, signant ainsi une recherche d'armatures acuminées. La réalisation fréquente d'un bordage proximal du bord opposé au dos pourrait être lié au calibrage ou au mode d'emmanchement de ces objets (fig. 12). Les microlamelles présentent un pic des longueurs autour de $10-15 \mathrm{~mm}$ et un gabarit calibré de 2-3 mm de largeur pour $1 \mathrm{~mm}$ d'épaisseur. À ce morphotype s'ajoutent des lamelles au dos latéralisé à droite dans $65 \%$ des cas. Ces pièces, parfois appointées, présentent des longueurs centrées autour de $25 \mathrm{~mm}$ et un gabarit moyen de 3-4 $\mathrm{mm}$ de largeur pour $2 \mathrm{~mm}$ d'épaisseur. Les microlamelles proviennent de débitages à table carénée orientée de diverses manières : sur front dorsal ou ventral déjeté (fig. 13; Lenoir et al., 1991; Langlais et al., 2007). Les lamelles, moins nombreuses, sont extraites de nucléus sur tranche d'éclats ou pyramidaux sur blocs, débités de manière unipolaire convergente, qui annoncent en quelque sorte l'ensemble supérieur (voir ci-dessous). Nous avons vu que plusieurs pièces dites « à encoches ou denticulées », correspondant en fait à des préformes de nucléus carénés, documentent les premières étapes d'installation des convexités depuis les flancs du nucléus par l'extraction de petits éclats lamellaires à la pierre (fig. 14). L'ensemble des productions (micro)lamellaires est réalisé de manière autonome vis-à-vis des débitages domestiques.

La composition de l'armement lithique de l'ensemble supérieur est très différente de celui du niveau sousjacent (tabl. 2). Elle se marque par une diversité typologique des lamelles à dos simples, appointées, tronquées ou denticulées, portant fréquemment des stigmates d'impact. Le groupe des simples réunit de nombreux fragments mésiaux difficiles à attribuer typologiquement. Le morphotype dominant - utilisé comme marqueur archéostratigraphique - est une lamelle à dos tronquée de manière oblique : la lamelle scalène (fig. 15, $\mathrm{n}^{\text {os }} 1$-14; voir historique in Langlais, 2008), anciennement dénommée " tête de brochet » (Cheynier, 1965) et parfois confondue avec des triangles (Peyrony, 1936). Quelques-unes sont denticulées (fig. 15, $\mathrm{n}^{\text {os }} 15-17$ ). Les lamelles scalènes de $\mathrm{SG}$ ne dérogent pas à la règle magdalénienne : elles présentent une latéralisation préférentielle (>95\%) $\mathrm{du}$ couple dos-troncature (dos dextre pour une troncature proximale et senestre pour une troncature distale), signant bien l'idée d'une véritable recette de fabrication. Les longueurs sont comprises entre 25 et $35 \mathrm{~mm}$ pour des gabarits stables, de 4-6 $\mathrm{mm}$ de largeur pour 2-3 $\mathrm{mm}$ d'épaisseur. Bien que certaines extrémités laissées brutes ou presque correspondent à des surfaces de type piquanttrièdre (fig. $15, \mathrm{n}^{\circ} 7$ ) et que deux microburins aient été reconnus dans la série, il demeure délicat de préciser si l'emploi de ce procédé de réalisation a été systématique à $\mathrm{SG}$, comme cela l'a été dans d'autres séries (Langlais, 2007a). La présence de stigmates d'impact localisés sur l'extrémité opposée à la troncature (fig. $15, \mathrm{n}^{\text {os }} 3$ et 11) pourrait permettre d'envisager une orientation de la troncature en partie basale des barbelures lithiques. Toutefois, ces observations demandent à être approfondies. Les autres lamelles à dos tronquées, moins nombreuses, se distinguent par une délinéation rectiligne à concave des troncatures. Elles présentent la même latéralisation préférentielle que les lamelles scalènes. Leur longueur varie entre 30 et $40 \mathrm{~mm}$ pour des gabarits identiques aux lamelles scalènes. Il pourrait s'agir d'une simple variabilité de la délinéation des troncatures. Les lamelles à dos denticulées, appointées ou étroites ne présentent par contre pas de latéralisation préférentielle des dos ou de l'extrémité acuminée. Tandis que la plupart des pièces denticulées reflète le même gabarit que les lamelles scalènes, d'autres lamelles à dos offrent des gabarits 

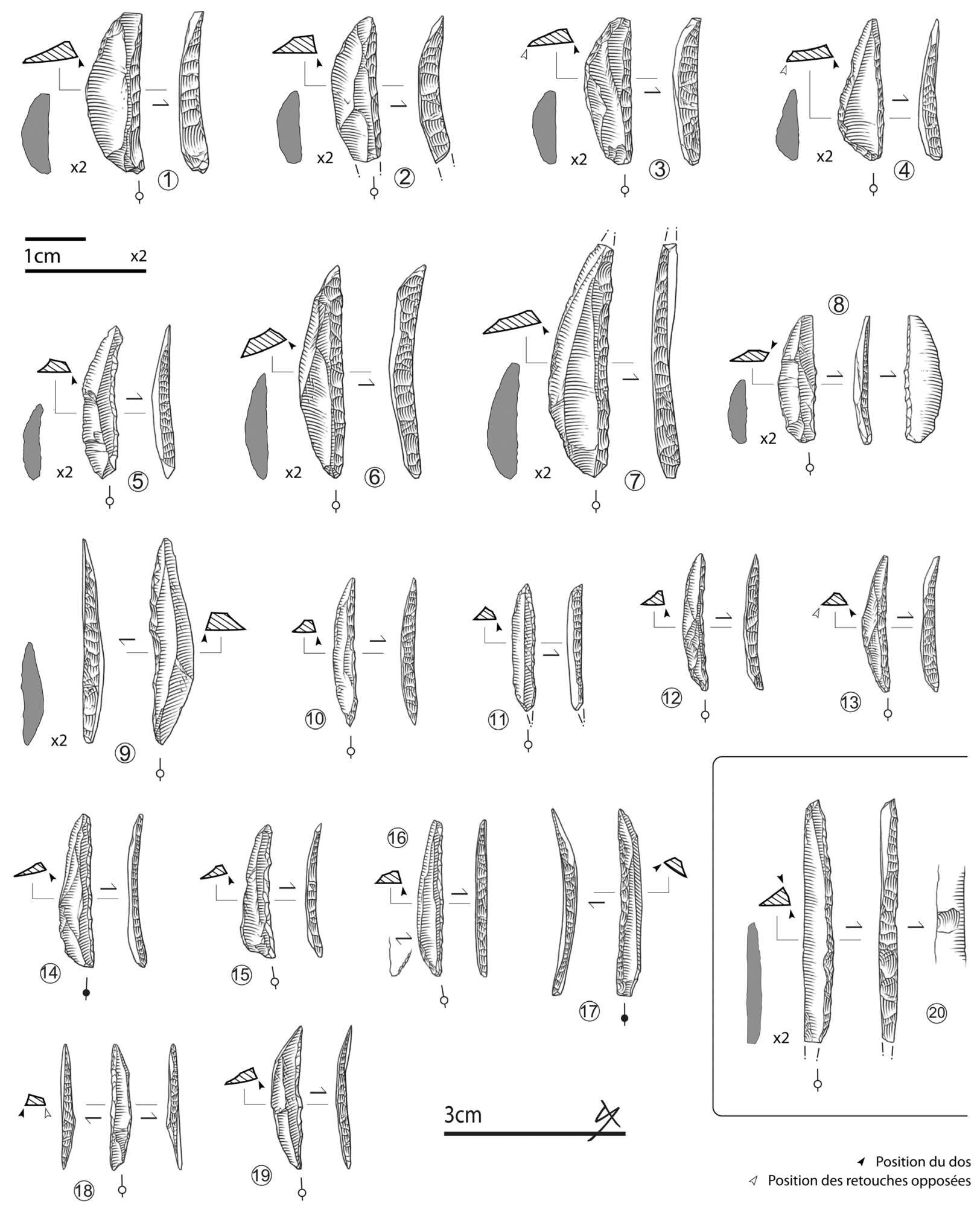

Fig. 12 - SG (ensemble inférieur) : exemples de microlamelles à dos (dessins S. Ducasse).

Fig. 12 - SG (lower stratigraphic unit): examples of backed microbladelets (drawings S. Ducasse). 

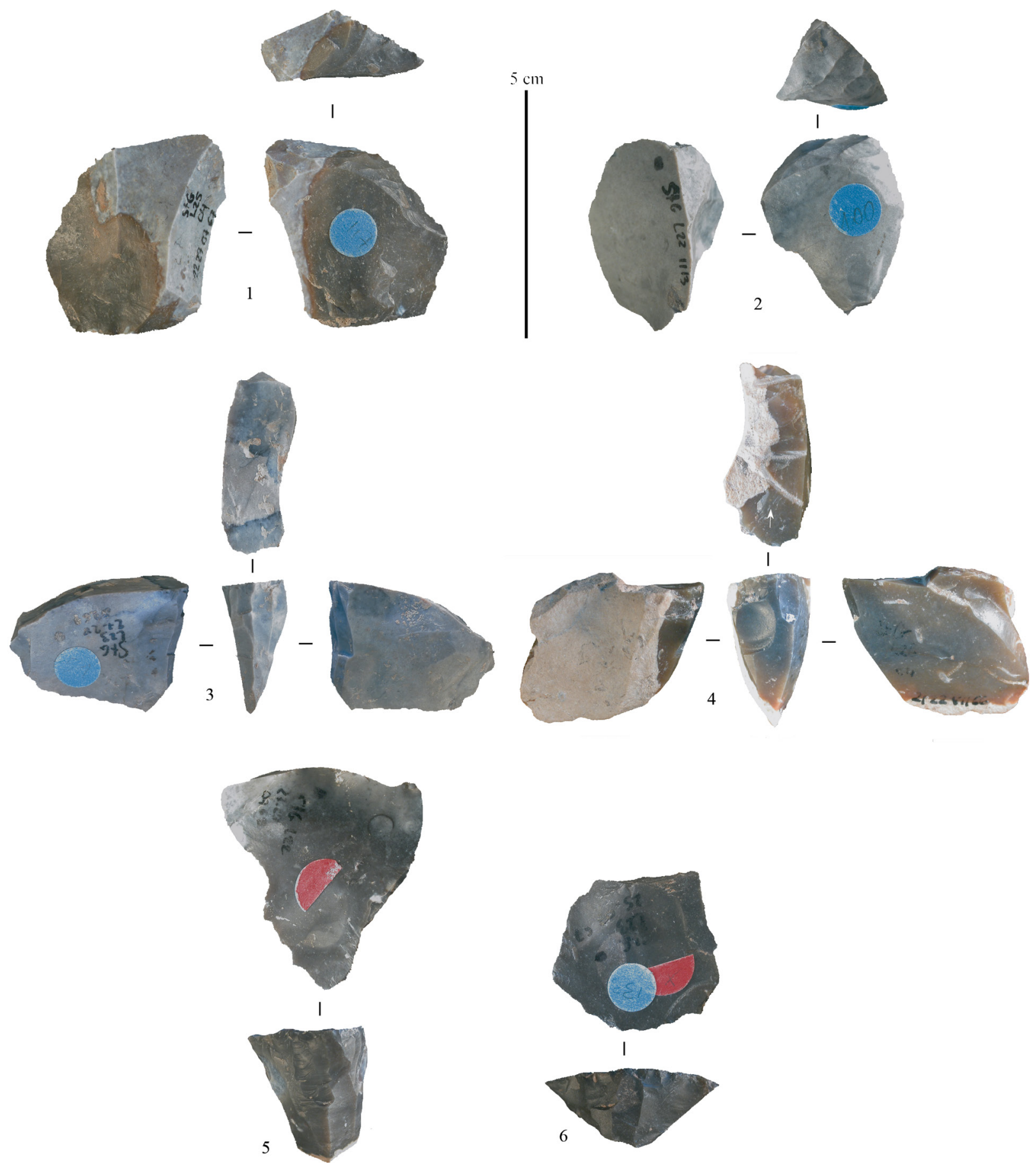

Fig. 13 - SG (ensemble inférieur) : exemples de nucléus carénés. 1-2 : front ventral déjeté ; 3-4 : sur tranche; 5-6 : front dorsal (DAO ML).

Fig. 13 - SG (lower stratigraphic unit): examples of carinated core (CAD ML).

autour de 3-4 mm de largeur pour $1 \mathrm{~mm}$ d'épaisseur. Les lamelles à dos simples, tronquées, denticulées ou appointées (fig. 15) proviennent des mêmes schémas opératoires de type unipolaire convergent sur petits rognons ou éclats (fig. 16). En revanche, les lamelles étroites sont issues préférentiellement de débitages sur tranche d'éclats. Dans les deux cas, les productions lamellaires sont totalement autonomes vis-à-vis des productions domestiques.

\section{Éléments de comparaison}

Les deux ensembles se distinguent bien par leur contenu lithique, tant dans l'outillage et ses procédés de fabrication que dans la confection des armatures de chasse. Tandis que les lamelles à dos simples sont présentes au tout au long de la séquence, l'existence de marqueurs chronostratigraphiques permet de distinguer les deux ensembles et d'identifier d'éventuels problèmes stratigraphiques. Ainsi, la 

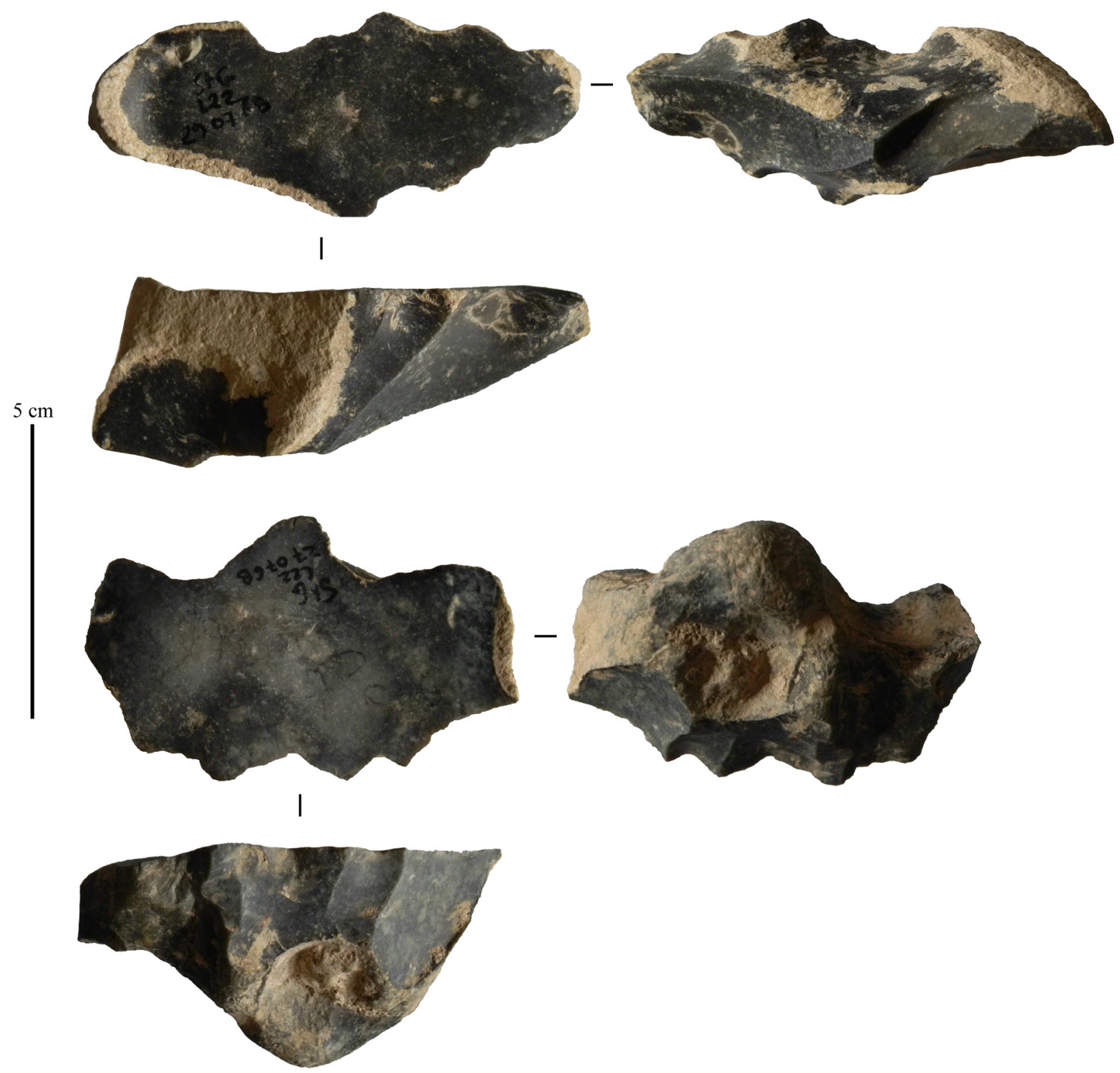

Fig. 14 - SG (ensemble inférieur) : exemples d'ébauches de nucléus carénés (DAO ML).

Fig. 14 - SG (lower stratigraphic unit): examples of carinated cores preforms (CAD ML).

présence très limitée de lamelles scalènes dans l'ensemble inférieur et de microlamelles à dos dans l'ensemble supérieur (tabl. 2) signe vraisemblablement des mélanges, inévitables dans ce type de contexte malgré le nouveau découpage proposé. Il en est de même possiblement pour certaines lames fortement retouchées ou appointées et supports laminaires débités au percuteur tendre organique, bien documentés dans l'ensemble supérieur et plus rares dans l'ensemble sous-jacent. Si deux tendances se dégagent entre ces deux temps du Magdalénien, certains éléments, comme l'exploitation des silex locaux de qualité, la dissociation des productions domestiques et cynégétiques ou la mise en œuvre de débitages lamellaires identiques pour certains supports rectilignes plus épais, indiquent une continuité technique entre les deux.

L'ensemble inférieur livre une industrie attribuable au Magdalénien inférieur. La relative variabilité morphomé- trique - bien que de tendance laminaire prononcée - des supports d'outils domestiques extraits préférentiellement à la pierre, l'importante composante (micro)lamellaire et l'absence de raclettes constituent en effet des éléments de rapprochement avec plusieurs gisements de la même période. La comparaison des armatures de SG avec d'autres séries du Magdalénien inférieur permet néanmoins de souligner une variabilité interne à cette période. Nous remarquons ainsi l'absence à $\mathrm{SG}$ de pointes à cran - documentées à Gandil, Tarn-et-Garonne, c. 20 (Ladier, 2000) et à Fontgrasse, Gard (Bazile et Monnet-Bazile, 2000) - l'extrême rareté des (micro)lamelles à dos dextre marginal - présentes aux Scilles, Haute-Garonne (Pétillon et al., 2008; Langlais et al., 2010), au Petit Cloup Barrat, Lot, c. 4 (Langlais, 2010; Ducasse et al., 2011) ou au Taillis des Coteaux, Vienne, AG-IIIc (Primault com. pers.; Langlais obs. pers. et master en cours de A. Gioé 

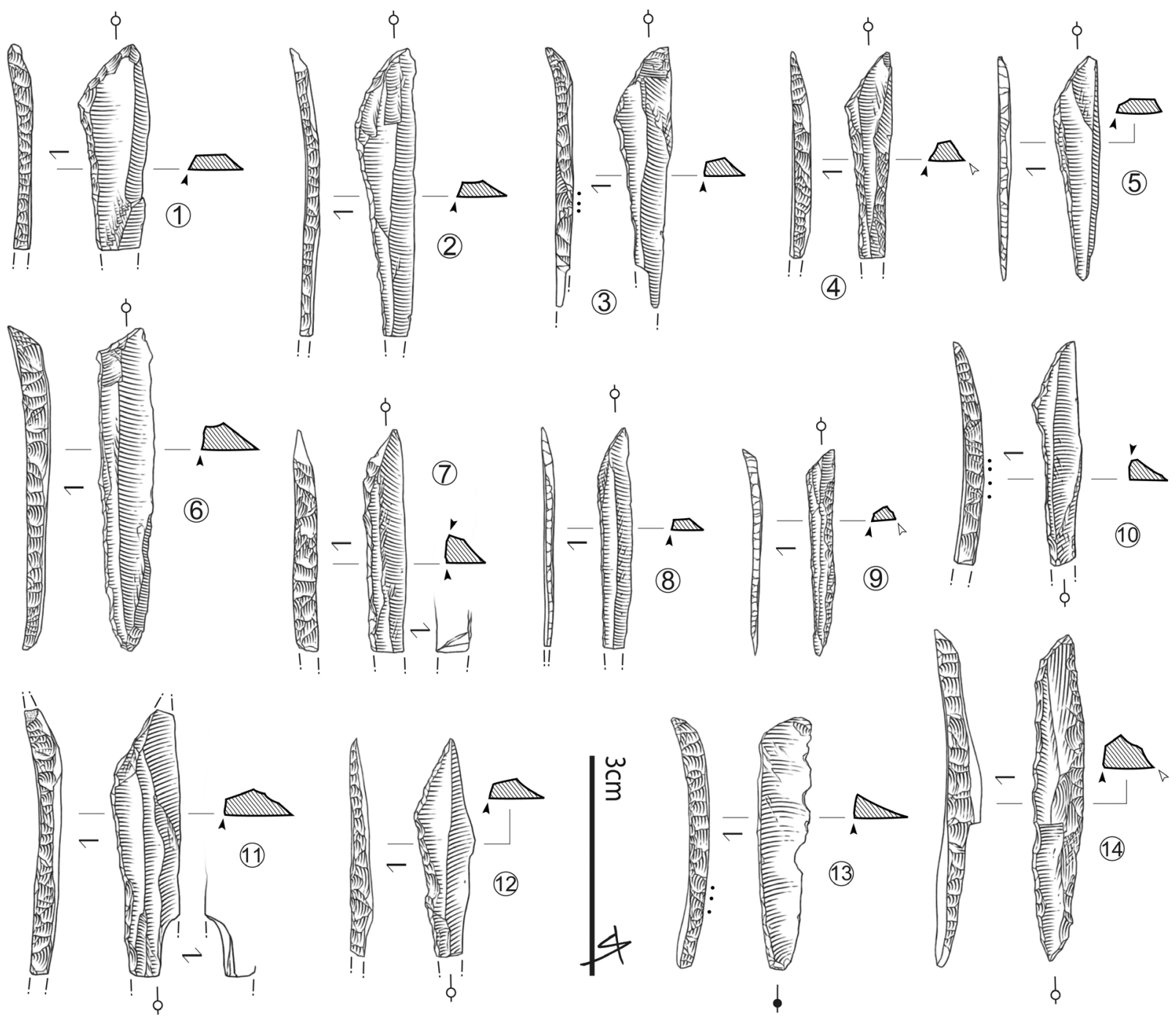

1 Position du dos (section)
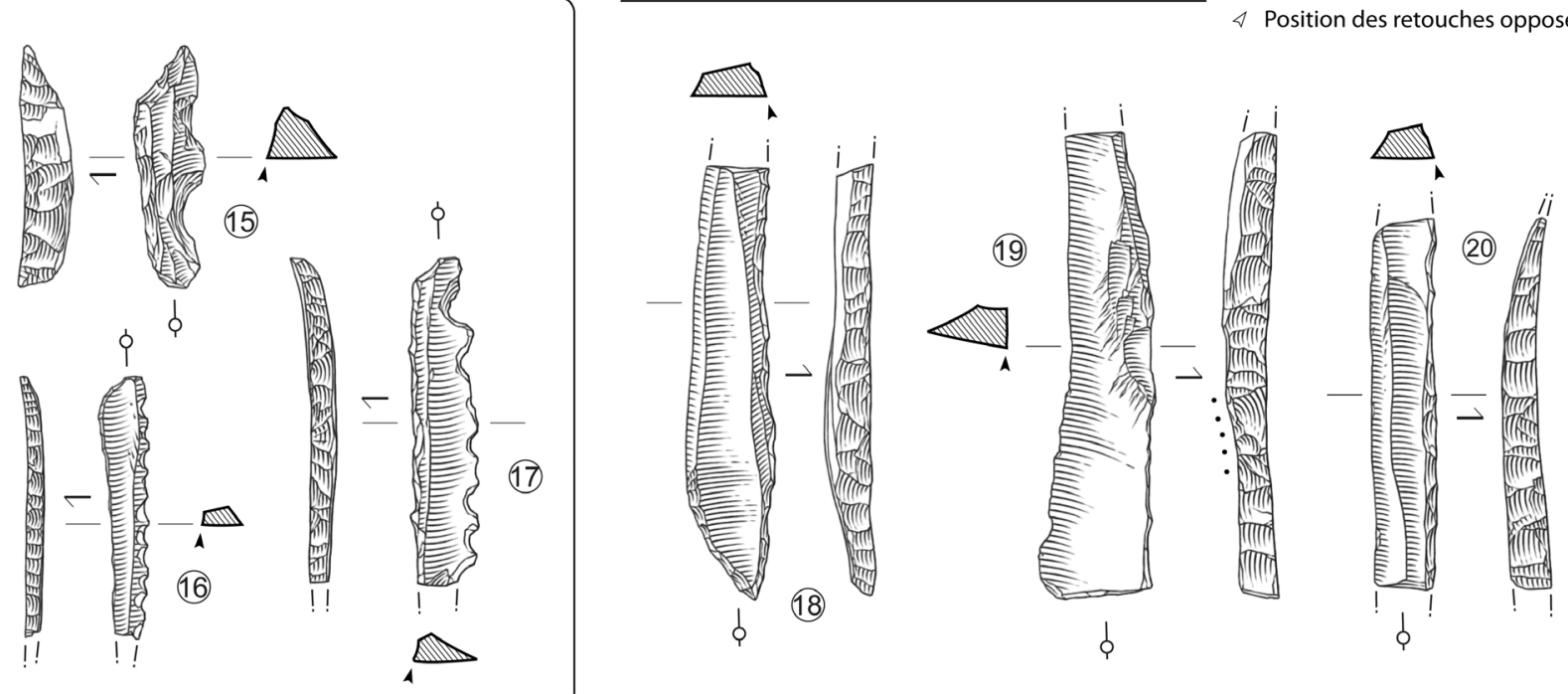

Fig. 15 - SG (ensemble supérieur) : exemples de lamelles scalènes dont denticulées et de grandes lamelles à dos (dessins S. Ducasse).

Fig. 15 - SG (upper stratigraphic unit): examples of scalene bladelets, including some denticulated ones, as well as large backed bladelets (drawings $S$. Ducasse). 

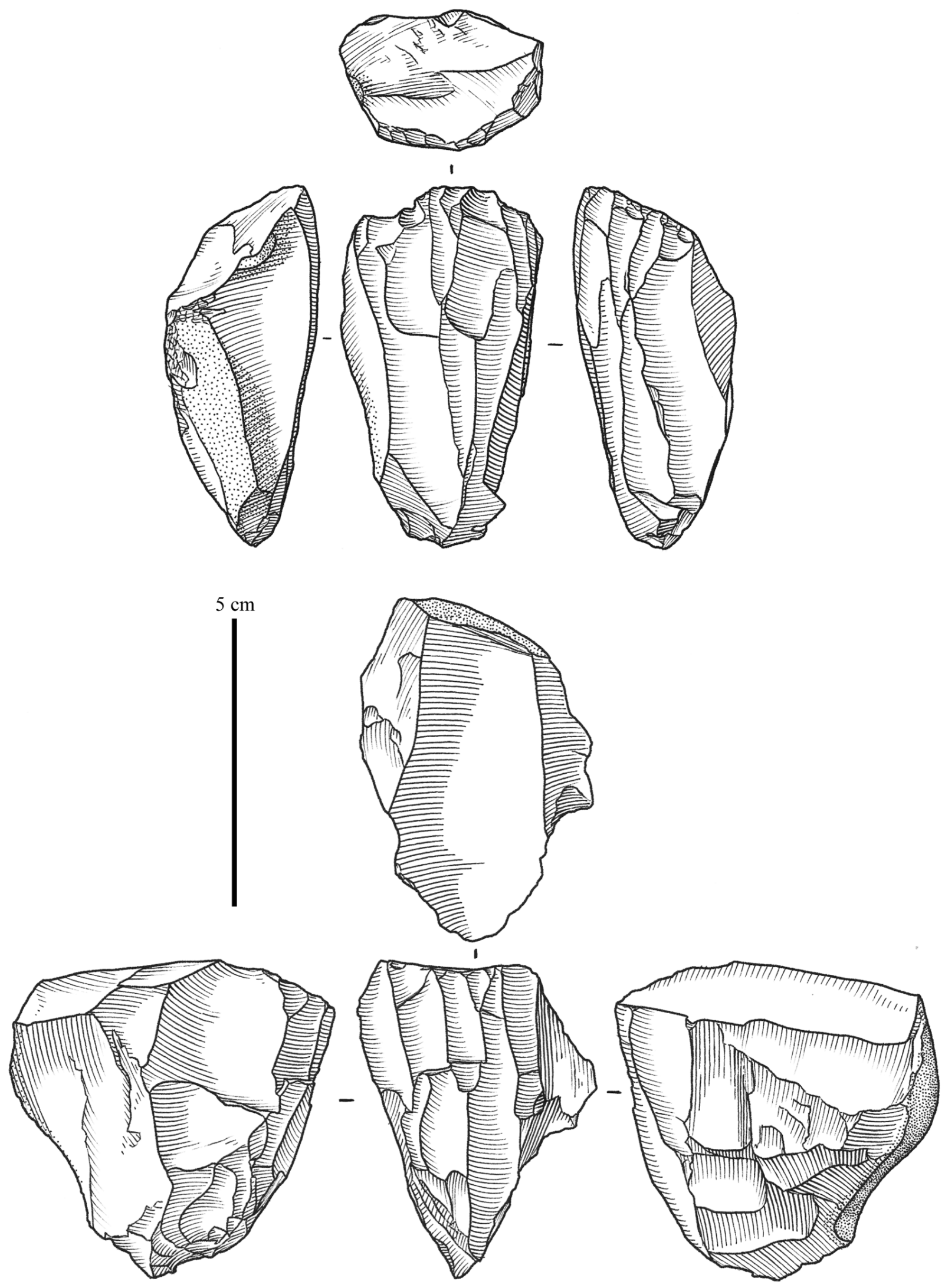

Fig. 16 - SG (ensemble supérieur) : exemples de nucléus à lamelles (dessins S. Pasty).

Fig. 16 - SG (upper stratigraphic unit): examples of bladelet cores (drawings S. Pasty). 
à l'université Toulouse 2) - et très faible nombre de microlamelles à dos inverse (fig. 12, no 8 ) - morphotype dominant dans les séries de Fontgrasse ou Montlleó en Cerdagne (Langlais, 2007a). Le Magdalénien inférieur de SG se caractérise plutôt par une monotonie typologique avec une préférence pour des microlamelles aux dos directs envahissants, préférence qui rappelle le Taillis des Coteaux AG-IIIa (Primault et al., 2007), Gandil c. 23-25 (Langlais et al., 2007) et éventuellement Thèmes, Yonne (Lebrun-Ricalens et Brou, 2003).

Pour l'ensemble supérieur, attribuable au Magdalénien moyen, la production laminaire réalisée au percuteur tendre organique permet une standardisation des supports à vocation domestique et un débitage de grandes lamelles qui fournissent les supports d'armatures. Parmi celles-ci, le morphotype " lamelle scalène » offre plusieurs points de comparaison dans le Sud-Ouest. C'est le cas par exemple en Dordogne à Chancelade (fouilles Bouyssonie : Cheynier et Bouyssonie, 1955) ou au Flageolet II c. IX (Rigaud, 1979) et dans le Lot à CombeCullier (Adachi, 2000), au Petit Cloup Barrat, sommet c. 4 et niveaux supérieurs (Langlais, 2010; Ducasse et al., 2011) et aux Peyrugues, c. 3 (Langlais, 2007a) ou encore au Martinet, Lot-et-Garonne, c. 4 (Le Tensorer, 1981). La répartition géographique des lamelles scalènes et le fait qu'elles soient tantôt associées aux séries attribuées au Magdalénien « à navettes » ou « à pointes de LussacAngles » et tantôt dissociées, mériteront une attention particulière (voir ci-dessous).

\section{L'INDUSTRIE OSSEUSE}

T industrie osseuse des fouilles Trécolle comprend 330 pièces réparties en 263 objets en bois de cervidé et 67 en os. Nous laissons à part vingt-huit pièces de l'ensemble médian et dix-huit de provenance indéterminée. Les éléments en bois de cervidé dont l'espèce a pu être déterminée se rapportent tous au renne. Aucun élément en ivoire de mammouth ou en os de cétacé n'a été identifié ; les éléments de parure en matière osseuse, déjà décrits dans une autre publication (Vanhaeren et D'Errico, 2003), ne seront pas évoqués ici. L'état de conservation de l'industrie osseuse est globalement médiocre. Les surfaces sont souvent altérées (fissuration, desquamation) et la fragmentation postdépositionnelle est particulièrement intense puisqu'elle affecte $78 \%$ des pièces en os et $87 \%$ des pièces en bois de cervidé - une situation qui a représenté un sérieux handicap lors de l'analyse. Une recherche systématique a cependant permis de trouver quarante-trois raccords et de reconstituer partiellement trente objets. Par ailleurs, les pièces des ensembles inférieur et supérieur se distinguent par une nette différence de patine : claire et rougeâtre pour l'ensemble inférieur, sombre et noirâtre pour l'ensemble supérieur; de plus, seules $2 \%$ des pièces de l'ensemble inférieur présentent des traces de concrétion, alors que ce caractère se retrouve sur $23 \%$ des pièces de l'ensemble supérieur.

\section{L'industrie en bois de cervidé}

L'industrie en bois de cervidé de l'ensemble inférieur a déjà été en partie présentée dans une autre publication (Pétillon et Ducasse, 2012), antérieure à la réévaluation archéostratigraphique, ce qui explique les différences d'effectif avec le présent texte. Le schéma opératoire dominant dans cet ensemble est la production d'objets sur baguettes extraites par double rainurage longitudinal (tabl. 3). Parmi les huit déchets se rapportant à ce type de débitage, cinq témoignent de l'exploitation de la perche A et de l'andouiller de glace de bois de gros module (les deux seules parties basilaires correspondant à un bois de chute et à un possible bois de massacre). Le nombre de

\begin{tabular}{|l|l|c|c|c|}
\multicolumn{2}{|c|}{} & Inférieur & Supérieur & Total \\
\hline \multirow{4}{*}{ Déchets de débitage } & d. DR longitudinal & 8 & 4 & $\mathbf{1 2}$ \\
\cline { 2 - 5 } & élagage digitation par DR & 2 & 1 & $\mathbf{3}$ \\
\cline { 2 - 5 } & d. transversal par entaillage & 3 & 3 & $\mathbf{6}$ \\
\cline { 2 - 5 } & d. par percussion lancée & 3 & 0 & $\mathbf{3}$ \\
\hline Supports (baguettes) extraites par DR & 16 & $9(+2 *)$ & $\mathbf{2 7}$ \\
\hline Déchets de façonnage sur baguette & 14 & 4 & $\mathbf{1 8}$ \\
\hline Ébauches de pointe & & 0 & 1 & $\mathbf{1}$ \\
\hline \multirow{4}{*}{$\begin{array}{l}\text { Objets finis } \\
\text { sur baguette }\end{array}$} & outils intermédiaires & 3 & 6 & $\mathbf{9}$ \\
\cline { 2 - 5 } & alênes & 4 & $0(+3 *)$ & $\mathbf{7}$ \\
\cline { 2 - 5 } & pointes de projectile & 47 & $19(+1 *)$ & $\mathbf{6 7}$ \\
\cline { 2 - 5 } & indéterminés & 26 & 10 & $\mathbf{3 6}$ \\
\hline \multirow{3}{*}{$\begin{array}{l}\text { Objets finis sur } \\
\text { support en volume }\end{array}$} & bâtons percés & 2 & 1 & $\mathbf{3}$ \\
\cline { 2 - 5 } & outils mousses & 15 & $0(+2 *)$ & $\mathbf{1 7}$ \\
\cline { 2 - 5 } & pointes & 0 & 1 & $\mathbf{1}$ \\
\hline Indéterminés & & $\mathbf{1 5 2}$ & $\mathbf{6 4}\left(+\mathbf{8}^{*}\right)$ & $\mathbf{2 2 4}$ \\
\hline \multirow{2}{*}{\begin{tabular}{l} 
Total bois de cervidé \\
\cline { 2 - 5 }
\end{tabular}} & & & & $\mathbf{1 4}$ \\
\hline
\end{tabular}

Tabl. 3 - SG (Trécolle) : composition techno-typologique de l'industrie en bois de cervidé par ensemble (d. : débitage; DR : double rainurage; * : pièces dont la patine et la typologie évoquent l'ensemble inférieur).

Table 3 - SG (Trécolle): Technotypological list of antler industry, organized by unit (d: debitage; DR: groove and splinter technique; *: artefacts whose patina and typology suggest the lower stratigraphic unit). 

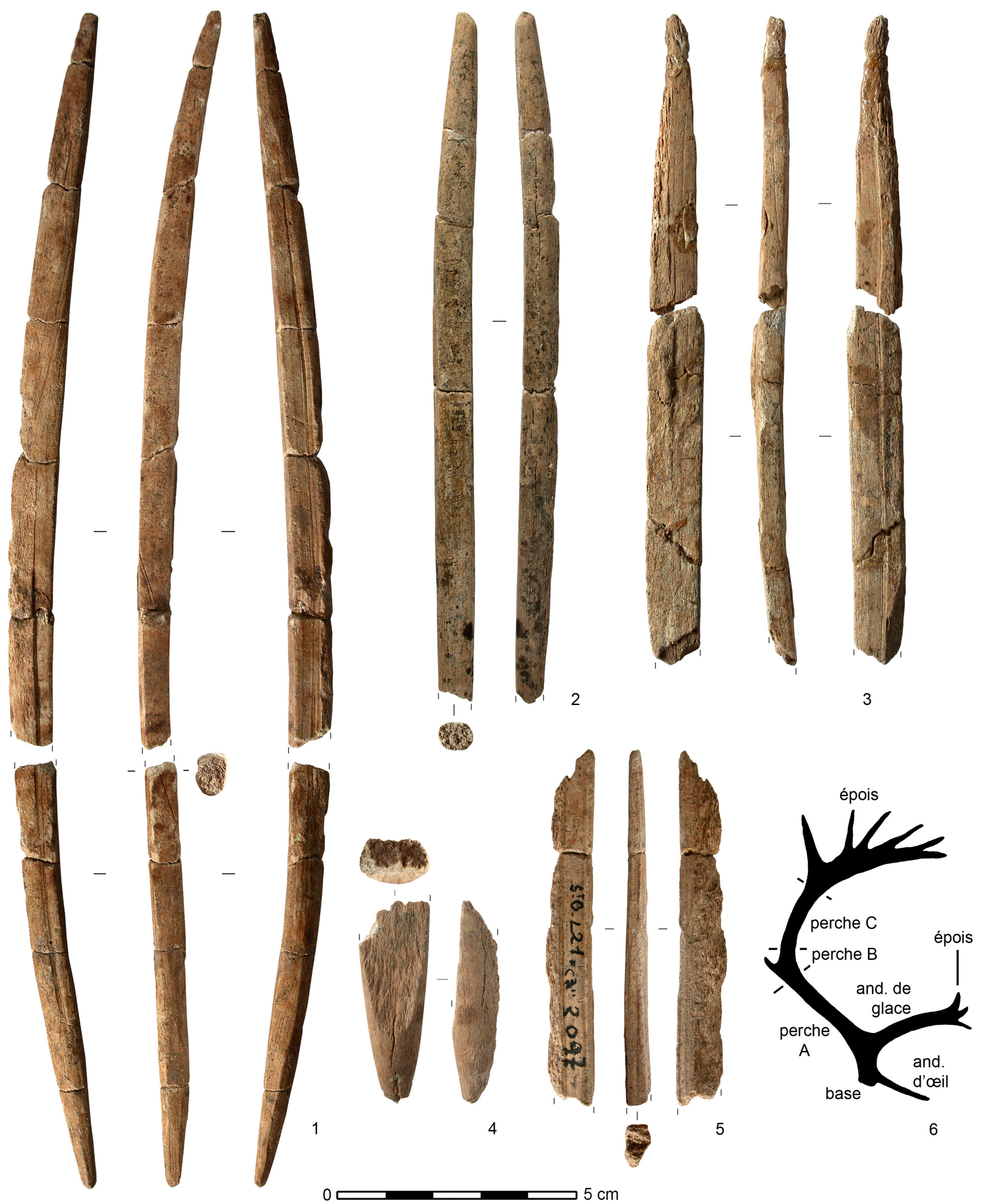

4

5

6

Fig. 17 - SG (ensemble inférieur) : industrie en bois de cervidé : baguettes. 1-3, $5:$ baguettes de petit calibre présentant des traces d'extraction par double rainurage $\left(\mathrm{n}^{\mathrm{os}} 1,3,5\right)$ ou ayant déjà subi un début de mise en forme par raclage $\left(\mathrm{n}^{\circ} 2\right.$ et une partie de la pièce $\mathrm{n}^{\circ} 1$ ); 4 : extrémité de baguette de gros calibre (DAO JMP); 6 : rappel de la terminologie utilisée pour la description des bois de renne.

Fig. $17-S G$ (lower stratigraphic unit): antler industry: splinters. 1-3, 5: small diameter splinters showing traces of extraction by the groove and splinter technique (nos. 1, 3, 5) or having already been shaped by scraping (no. 2 and part of no. 1); 4: the extremity of a thicker diameter splinter (CAD JMP); 6: terminology used for the description of reindeer antlers. 
baguettes extraites de chaque bloc est de un (trois cas) ou deux (deux cas); ces baguettes sont larges de 12 à $15 \mathrm{~mm}$, avec un tissu compact épais de 6 à $8 \mathrm{~mm}$. Le détachement de leurs extrémités est effectué par entaillage transversal. L'exploitation des parties hautes de la perche (perche B ou C) est documentée par deux déchets d'élagage attestant du sectionnement d'épois par double rainurage opposé - type de déchet que nous rapportons également à un schéma d'exploitation longitudinale de la perche.

Les supports sont présents sous la forme de seize baguettes montrant sur leurs bords des traces d'extraction par double rainurage, même si certaines ont déjà subi un début de mise en forme par raclage (fig. 17). Aucune n'est entière, mais les extrémités intactes sont systématiquement appointées et les trois plus grands fragments montrent l'existence de supports longs et effilés (longueur de 138 à $242 \mathrm{~mm}$ pour une largeur de 6 à $8 \mathrm{~mm}$ : fig. 17, $n^{\circ} 1$ ), même si des éléments de plus gros calibre sont également présents (fig. 17, n ${ }^{\circ} 4$ ). Une série de quatorze déchets « à base raccourcie » atteste du façonnage (mise à l'axe, mise à longueur) ou de la réfection d'objets sur baguette (Chauvière et Rigaud, 2005 et 2008). Enfin, sur les quatre-vingts fragments d'objets finis sur baguette, cinquante-quatre ont pu être déterminés typologiquement et se répartissent en trois catégories (tabl. 4) :

- trois outils intermédiaires (type coin ou ciseau), dont deux courts fragments distaux et un exemplaire entier (fig. 18, $\mathrm{n}^{\circ} 1$ );

- quatre « alênes », terme par lequel nous désignons des aiguilles à chas en bois de cervidé, de plus grandes dimensions que leurs homologues en os (calibre moyen : $5,6 \times 4,9 \mathrm{~mm}$; aucune n'est entière mais les trois plus longs fragments mesurent entre 60 et $95 \mathrm{~mm}$ ) et au profil parfois courbe. Un seul exemplaire présente encore une portion de chas (fig. 18, $\mathrm{n}^{\circ} 2$ ). En raison de la forte fragmentation de l'industrie en bois de cervidé, le nombre de pièces ayant pu être attribuées à cette catégorie typologique est faible, mais il faut souligner que dix-huit des vingt-six « objets sur baguette indéterminés » sont de possibles fragments d'alênes;

- quarante-sept fragments de pointes de projectile. Dans cet ensemble d'armatures, le seul type clairement identifié est la pointe à base pleine (quatre exemplaires : fig. 18, $\mathrm{n}^{\text {os }} 4,5,6$ et 9). La longueur des plus grands fragments permet d'estimer que les pointes complètes ne devaient pas dépasser 100 à $120 \mathrm{~mm}$ de long. Leur section est généralement ovale ou subquadrangulaire et leur calibre est réduit (moyenne : 8,0 × 6,6 mm). Environ $40 \%$ de ces pointes présentent une rainure longitudinale (seize cas, en général sur la face supérieure ou inférieure), exceptionnellement deux (trois cas).

Les techniques de débitage du bois de renne selon l'axe transversal sont mal documentées; seules quelques pièces témoignent du sectionnement de la perche et des andouillers d'œil et de glace de bois de module moyen et gros par entaillage bifacial. L'exploitation transversale du bois de renne est en fait attestée essentiellement sous la forme d'objets finis façonnés sur support en volume :

- deux fragments distaux de bâtons percés sur bois de module moyen (fig. 19, $\mathrm{n}^{\text {os }} 1,2$ ). Leur partie mésio-proximale (« manche » du bâton) est absente mais était manifestement constituée de l'andouiller de glace; ce choix est rare sur les bâtons percés magdaléniens où cette partie est habituellement constituée de la perche $\mathrm{A}$. Un troisième fragment distal issu d'un bâton percé similaire, mais sur bois de gros module, a été trouvé hors stratigraphie;

\begin{tabular}{|c|c|c|c|c|c|c|c|}
\hline & Effectif & $\begin{array}{l}\text { dont pièces } \\
\text { entières }\end{array}$ & $\begin{array}{l}\text { dont fragments } \\
\mathrm{L}>30 \mathrm{~mm}\end{array}$ & Longueur & Largeur & Épaisseur & $\begin{array}{l}\text { Épaisseur } \\
\text { compacta }\end{array}$ \\
\hline \multicolumn{8}{|c|}{ Inférieur } \\
\hline Baguettes & 16 & 0 & 14 & $3 \times[138-242]$ & $11,1 \pm 4,1$ & $8,8 \pm 1,9$ & $5,7 \pm 1,3$ \\
\hline Déchets de façonnage & 14 & 7 & 2 & $35,1 \pm 14,0$ & $8,2 \pm 3,3$ & $6,0 \pm 1,9$ & $4,6 \pm 1,7$ \\
\hline Outils intermédiaires & 3 & 1 & 0 & 126 & 13,5 & 8,6 & 6 \\
\hline Alênes & 4 & 0 & 3 & $3 \times[60-95]$ & $5,6 \pm 0,8$ & $4,9 \pm 0,1$ & $4,6 \pm 0,1$ \\
\hline Pointes de projectile & 47 & 0 & 26 & $3 \times[65-101]$ & $8,0 \pm 1,3$ & $6,6 \pm 1,2$ & $5,4 \pm 1,0$ \\
\hline \multicolumn{8}{|c|}{ Supérieur } \\
\hline Baguettes & 9 & 1 & 3 & 96 et $3 \times[36-65]$ & $12,1 \pm 3,7$ & $8,5 \pm 2,3$ & $5,0 \pm 1,5$ \\
\hline Déchets de façonnage & 4 & 3 & 1 & $55,7 \pm 6,0$ & $8,9 \pm 2,5$ & $7,8 \pm 0,9$ & $6,0 \pm 0,7$ \\
\hline Outils intermédiaires & 6 & 4 & 2 & $94,7 \pm 9,1$ & $16,7 \pm 1,5$ & $9,9 \pm 0,5$ & $6,6 \pm 1,0$ \\
\hline Pointes de projectile & 19 & 1 & 11 & 102 et $3 \times[104-142]$ & $11,8 \pm 3,0$ & $7,2 \pm 1,5$ & $5,3 \pm 1,2$ \\
\hline
\end{tabular}

Tabl. 4 - SG (Trécolle) : dimensions en millimètres des produits sur baguette en bois de cervidé (moyenne \pm écart-type). Sur chaque ligne, la population utilisée pour les calculs est indiquée en gras : elle est constituée des pièces entières auxquelles ont été ajoutées, lorsque le nombre de pièces entières était trop faible, les fragments longs de plus de $30 \mathrm{~mm}$. Pour les longueurs des pièces fragmentaires, seule la fourchette de longueur des trois fragments les plus longs est indiquée.

Table 4 - SG (Trécolle): dimensions (in $\mathrm{mm}$ ) of artefacts made on antler splinters (average \pm standard deviation). In each row, the population used for the calculation is indicated in bold. This value consists of complete specimens to which fragments longer than $30 \mathrm{~mm}$ were added when the number of complete specimens was too low. For the lengths of fragmentary pieces, only the range of lengths of the three longest is given here. 


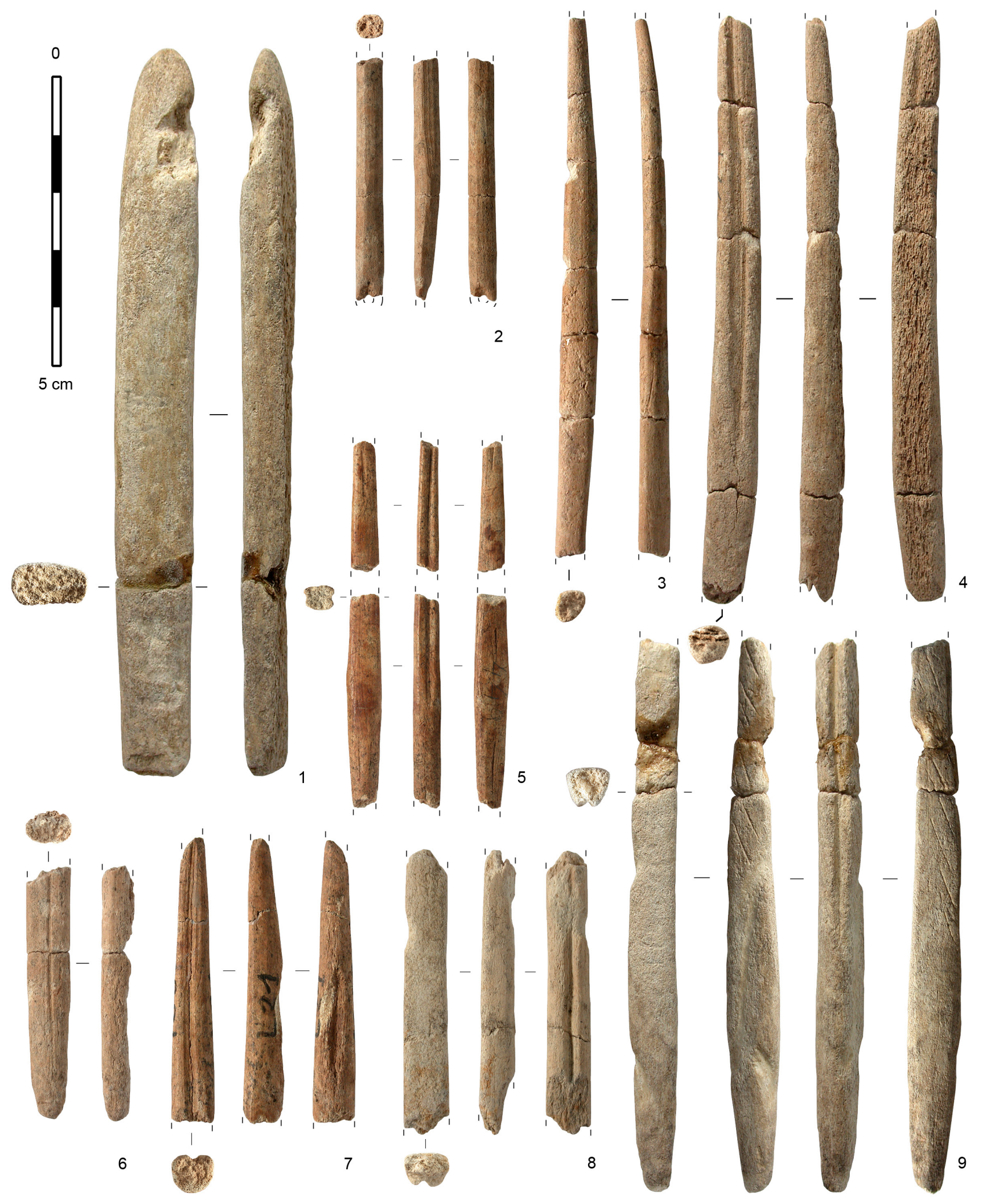

Fig. 18 - SG (ensemble inférieur) : industrie en bois de cervidé, objets finis sur baguette. 1 : outil intermédiaire; 2-3 : fragments mésiaux d'alênes; 4-9 : fragments de pointes de projectile avec rainures longitudinales (4-6 et 9 : pointes à base pleine). La pièce ${ }^{\circ} 5$ provient de l'ensemble médian mais présente la patine et la typologie des pièces de l'ensemble inférieur (DAO JMP).

Fig. 18 -SG : (lower stratigraphic unit): antler industry: finished objects made on splinters. 1: wedge; 2-3: mesial fragments of awls; 4-9: fragments of projectile points with longitudinal grooves (4-6 and 9: points with massive base). Artefact no. 5 was found in the middle part of the stratigraphy, but has the patina and the typological characteristics of pieces from the lower stratigraphic unit (CAD $J M P)$. 

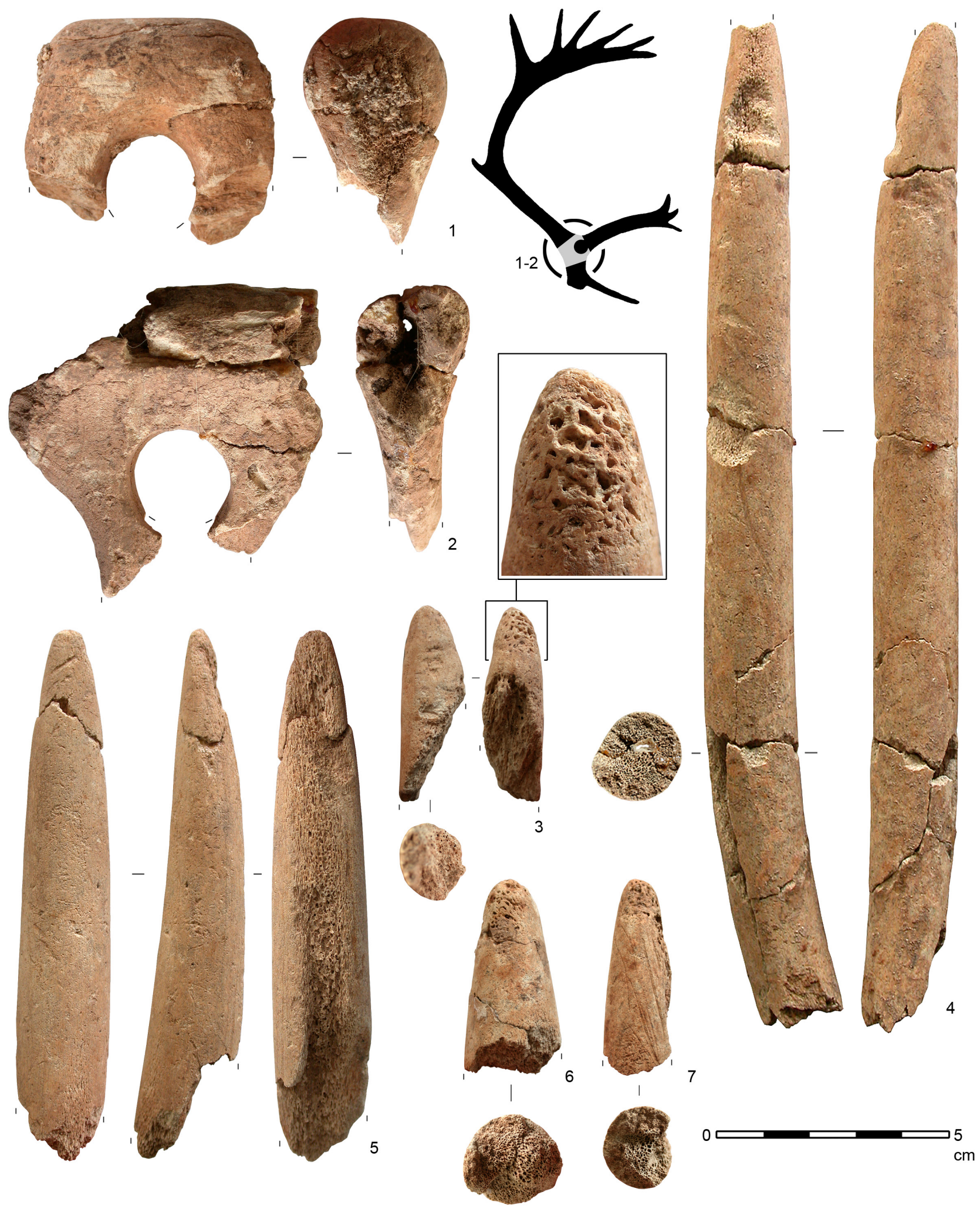

Fig. 19 - SG (ensemble inférieur) : industrie en bois de cervidé, objets finis sur support en volume. 1-2 : fragments distaux de bâtons percés, avec indication de leur emplacement sur le bois; 3-7 : fragments d'outils mousses sur épois (fragments distaux, sauf $n^{\circ} 4$ : fragment mésial); le détail de l'altération de l'extrémité distale figure pour la pièce nº 3 (DAO JMP).

Fig. 19 - SG (lower stratigraphic unit): antler industry, finished objects on complete antler segments. 1-2: distal fragments of perforated baton, with schematic indication of their position on the antler; 3-7: fragments of blunt tools made on tines (distal fragments, except no. 4: mesial fragment); detailed view of the alteration seen at the distal extremity of artefact no. 3 (CAD JMP). 
- quinze fragments d'outils mousses (fig. 19, $\mathrm{n}^{\text {os }} 3$ à 7). Il s'agit d'une série d'objets allongés, de section circulaire ou ovale et de dimensions assez importantes (le diamètre se situe généralement entre 20 et $23 \mathrm{~mm}$ et les plus grands fragments sont longs de 110 à $190 \mathrm{~mm}$ ), façonnés par raclage sur des épois de grand module. Ils présentent une extrémité proximale plane (avec, dans un cas, des traces d'utilisation comme plan de frappe) et une extrémité distale arrondie, parfois affectée d'une usure macroscopique particulière (une altération marquée exposant la spongiosa : fig. 19, $\mathrm{n}^{\circ} 3$ ). Leur fonction demeure indéterminée.

Enfin, il faut également signaler la présence dans l'ensemble inférieur de deux éclats de bois de renne et d'un andouiller de glace de bois de module moyen détaché par percussion. Ces éléments sont trop peu nombreux pour être rattachés à un schéma opératoire précis et aucun objet fini issu de ce type de débitage n'a été identifié.

L'industrie en bois de cervidé de l'ensemble supérieur comprend soixante-douze pièces. Huit d'entre elles, situées stratigraphiquement à la base de cet ensemble, rappellent par leur patine et leur typologie les caractères de l'ensemble inférieur (il s'agit de trois probables fragments mésiaux d'alênes, deux fragments distaux d'outils mousses, deux fragments mésiaux de baguettes longues et fines et un fragment mésial de pointe); ces objets ne seront pas pris en compte dans la suite de la description. Les soixante-quatre pièces restantes sont dominées, comme dans l'ensemble inférieur, par les témoignages de la production d'objets sur baguettes extraites par double rainurage longitudinal (tabl. 3). Parmi les quatre déchets se rapportant à ce schéma opératoire, l'un d'entre eux montre l'exploitation de la perche A d'un bois de mas-
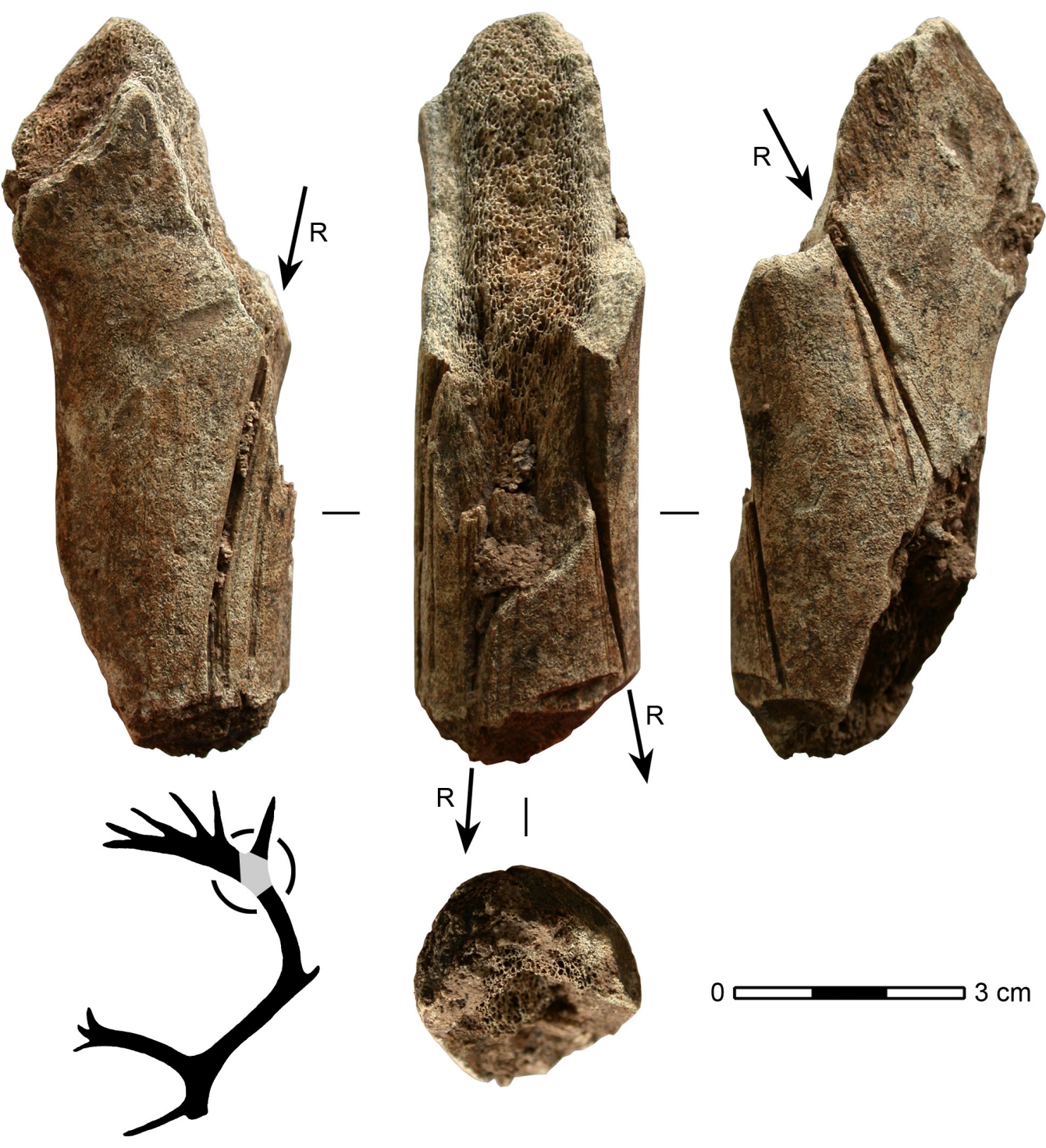

Fig. 20 - SG (ensemble supérieur) : industrie en bois de cervidé, déchet d'extraction de baguettes par rainurage longitudinal multiple sur bois de renne (perche C) avec indication de son emplacement sur le bois. R : rainure (DAO JMP).

Fig. 20 - SG (upper straigraphic unit): antler industry, waste product of reindeer antler (beam C) showing traces of splinter extraction by multiple longitudinal grooving with schematic indication of its placement on the antler. R: groove (CAD JMP). 
sacre de gros module, et deux autres proviennent respectivement d'une perche $\mathrm{B}$ et d'une perche $\mathrm{C}$ de module moyen ou gros. Les baguettes extraites sont larges de 10 à $20 \mathrm{~mm}$, avec un tissu compact épais de 5 à $7 \mathrm{~mm}$; le déchet sur perche $\mathrm{B}$ montre une extraction unique sur la face antérieure, tandis que le déchet sur perche $\mathrm{C}$ témoigne de trois extractions parallèles sur les faces médiale, antérieure et latérale (fig. 20). Les stigmates présents sur deux pièces indiquent que le détachement des extrémités des baguettes a été effectué par entaillage transversal. Ce petit ensemble est complété par un déchet d'élagage d'épois par double rainurage longitudinal opposé.

Six des neuf fragments de baguettes attribués à cet ensemble présentent encore sur un ou deux bords les traces du rainurage ayant servi à leur extraction. Les trois autres ont déjà subi un début de mise en forme par raclage. Quatre déchets de façonnage " à base raccourcie » et une ébauche de pointe témoignent du façonnage ou de la réfection sur place d'une partie de l'équipement. Enfin, parmi les trente-cinq objets finis sur baguette, vingt-cinq ont été typologiquement identifiés (tabl. 4) :

- six outils intermédiaires (type coin ou ciseau) façonnés sur des baguettes larges et épaisses (calibre moyen : $16,7 \times 9,9 \mathrm{~mm}$ ). Deux des quatre pièces complètes présentent un décor géométrique (croix, incisions obliques) situé sur la face supérieure et débordant dans un cas sur un bord (fig. $21, \mathrm{n}^{\text {os }} 1$ et 3 ). Un autre fragment d'outil du même type portant également un décor géométrique simple - une ligne en zigzag - a été trouvé hors stratigraphie, mais montre une patine rappelant celle de l'ensemble supérieur (fig. 21, $\mathrm{n}^{\circ}$ 2);

- dix-neuf pointes de projectile. Cinq d'entre elles ont une base amincie mais pas nettement biseautée, que nous avons dénommée « base en languette » (fig. 22, $\mathrm{n}^{\text {os }}$ 2, 4 et 5). Il s'agit là du mode d'emmanchement dominant dans cette série, même si un fragment de pointe à biseau simple (fig. 22, $\mathrm{n}^{\circ} 7$ ) et une pointe à biseau double sur support en volume (voir infra) ont également été identifiés. Les armatures de l'ensemble supérieur se caractérisent par une longueur parfois importante (l'unique pointe entière mesure $102 \mathrm{~mm}$, et 8 fragments renvoient à des objets qui, lorsqu'ils étaient entiers, devaient mesurer plus de $100 \mathrm{~mm}$, voire plus de 140-150 mm dans certains cas) et par une section large et plate (moyenne $11,8 \times 7,2 \mathrm{~mm}$ ), qui est dans la majorité des cas de forme ovale. Seules deux pièces présentent une rainure longitudinale; en revanche, trois montrent, sur la face supérieure ou sur un bord, des décors géométriques rappelant ceux des outils intermédiaires (fig. $22, \mathrm{n}^{\text {os }} 1$ et 2 ).

L'exploitation du bois de renne selon l'axe transversal est attestée par trois déchets (une base de bois de chute de module moyen, un tronçon de perche de gros module et une digitation) qui portent des traces de sectionnement par entaillage bifacial. Les objets sur support en volume se limitent à un petit fragment de bâton percé et une pointe à biseau double - la seule pointe de ce type dans la série, et également la seule à avoir été façonnée non sur un support plat (baguette) mais sur un épois (fig. 22, $\mathrm{n}^{\circ} 6$ ). L'ensemble supérieur n'a pas livré d'éclats de bois de cervidé ni d'autres pièces attestant d'un débitage par percussion lancée.

Enfin, même si l'industrie en bois de cervidé de l'ensemble médian ne sera pas présentée en détail, il faut rappeler que c'est de cet ensemble que provient l'une des deux pièces d'art mobilier figuratif des fouilles Trécolle : un fragment mésial de bâton percé gravé d'une représentation de cervidé (Marmier et Trécolle, 1973; Dubourg, 1997).

\section{L'industrie en os}

La plus grande part de l'industrie en os de l'ensemble inférieur (tabl. 5) se rapporte à la fabrication d'aiguilles, façonnées sur des baguettes extraites par double rainurage longitudinal. Les dix déchets documentant ce schéma opératoire montrent une grande variabilité, tant dans le choix du bloc osseux que dans le nombre d'extractions par bloc. Des traces de débitage par double rainurage sont ainsi visibles sur un os d'oiseau (un pan de rainure), un métatarsien II d'ours (une extraction inachevée : fig. 23, $\mathrm{n}^{\circ} 1$ ), un métatarsien de renne (une amorce de rainure), un éclat de diaphyse d'os long de grand ongulé et un probable métatarsien IV de cheval (dans les deux cas, extraction d'une baguette unique par double rainurage opposé : fig. 23, $\mathrm{n}^{\circ} 4$ ). Des extractions multiples (deux à quatre baguettes extraites de chaque bloc) sont attestées sur un humérus d'oie, un métacarpien de loup et un autre d'antilope saïga (fig. 23, $\mathrm{n}^{\text {os }} 2,3$ et 5). Dans tous les cas, les baguettes extraites sont larges de 2 à $4 \mathrm{~mm}$. Les produits de ce type de débitage sont présents sous la forme de deux fragments de baguettes en cours de façonnage (ébauches d'aiguilles), un déchet de façonnage de baguette avec traces de sectionnement transversal par sciage, et dix fragments d'aiguilles. Ces derniers, longs de 9 à $23 \mathrm{~mm}$, sont en général de section circulaire ou ovale (calibre moyen : 2,3 $\times 1,7 \mathrm{~mm}$ ) et les deux pièces dont la partie proximale est préservée montrent des traces de réfection du chas (percement d'un second chas après fracture du premier : fig. $23, \mathrm{n}^{\text {os }} 6$ et 7 ).

L'industrie en os de cet ensemble comprend également trois fragments de lissoirs sur hémicôte dont un présente une série d'incisions sur un bord (fig. 23, $n^{\text {os }} 8$ et 9); deux parties distales d'outils spatuliformes (plus étroits et plus épais que des lissoirs : fig. 23, $n^{\circ} 10$ ) dont un sur métatarsien de renne; et un troisième outil spatuliforme sur éclat de radius d'antilope saïga. Un éclat de diaphyse d'os long de grand ongulé montre à une extrémité des traces d'utilisation comme outil de percussion (« pilon »), et deux fragments d'os d'oiseau présentent des traces de raclage qui ne semblent pas se rapporter à des activités de boucherie.

L'industrie en os de l'ensemble supérieur est également dominée par les éléments se rapportant à la fabrication des aiguilles. Trois déchets témoignent de l'extraction de baguettes par double rainurage sur métapodien d'antilope saïga (fig. 24, $\mathrm{n}^{\circ} 1$ ) et sur tibia de la taille du renne (le nombre d'extractions n'est pas déterminable); un quatrième déchet atteste de l'extraction d'une unique 


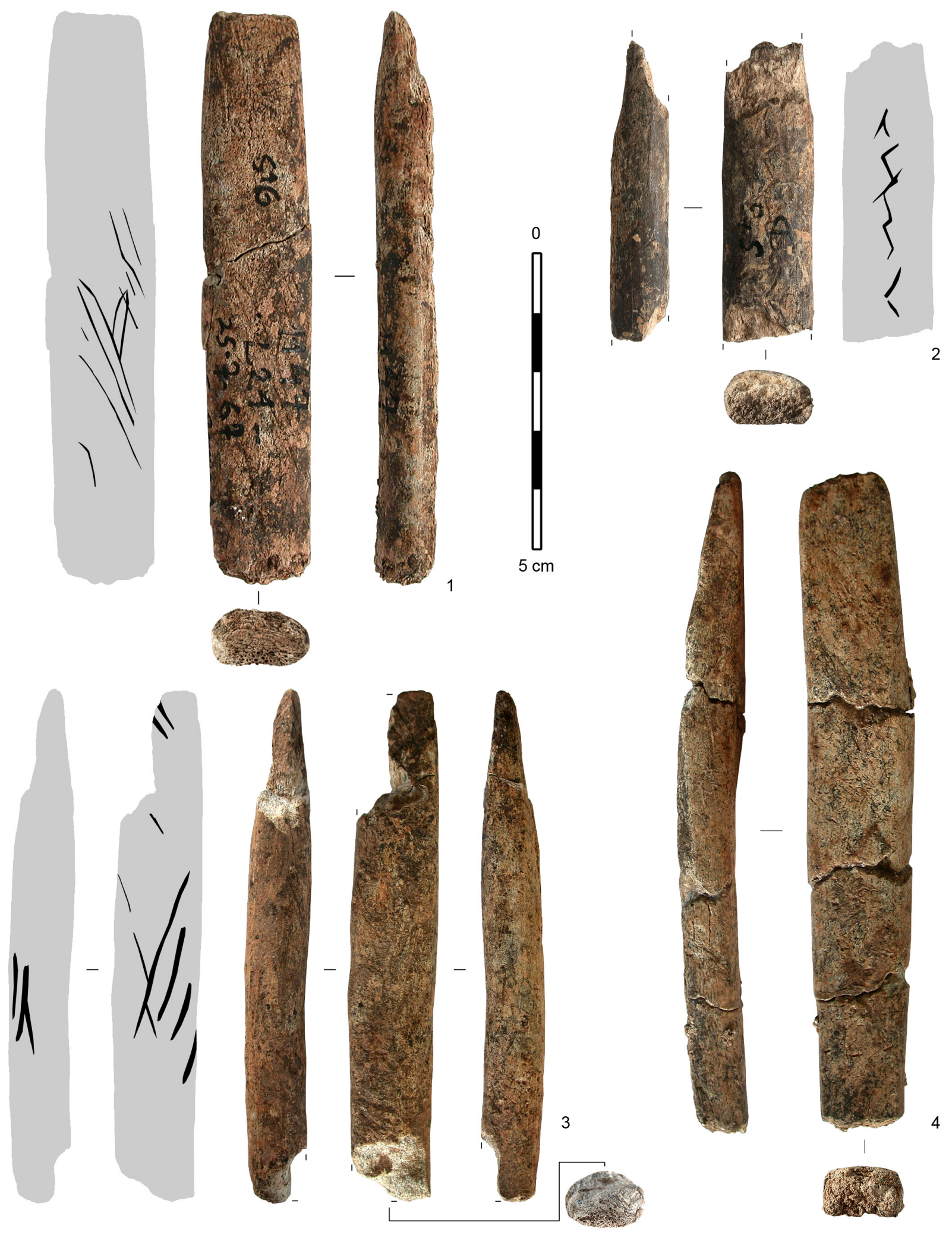

Fig. 21 - SG (ensemble supérieur) : industrie en bois de cervidé, outils intermédiaires sur baguette avec croquis sommaire des décors géométriques. 1, 3-4 : exemplaires entiers; 2 : fragment mésial découvert hors stratigraphie mais présentant la patine et la typologie des pièces de l'ensemble supérieur (DAO JMP).

Fig. 21 - SG (upper stratigraphic unit): antler industry, wedges made on splinters with rough sketch of the the geometric decoration. 1, 3-4: complete specimens; 2: mesial fragment not found in stratigraphic position, but having the patina and the typological characteristics of pieces from the upper stratigraphic unit (CAD JMP). 


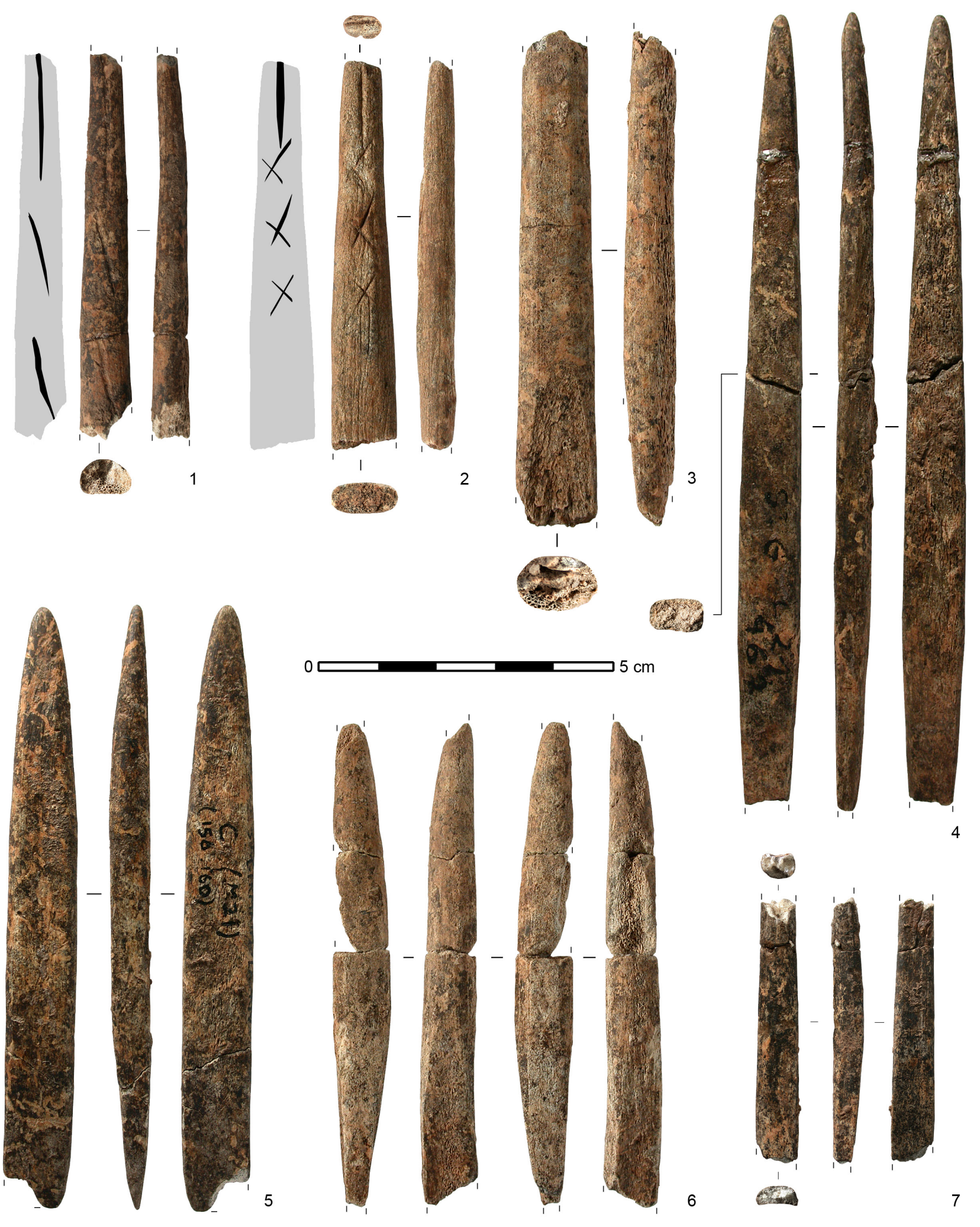

Fig. 22 - SG (ensemble supérieur) : industrie en bois de cervidé, pointes de projectile sur baguette (sauf $\mathrm{n}^{\circ} 6:$ sur épois) avec croquis sommaire des décors géométriques. 1,3 : pointes de type indéterminé (fragments mésiaux); 2, 4-5: pointes à base en languette; 6 : pointe à biseau double sur support en volume; 7 : pointe à biseau simple (DAO JMP).

Fig. 22 - SG (upper stratigraphic unit): antler industry, projectile points made on splinters (except no. 6: on tine) with rough sketch of the geometric decoration. 1, 3: points of indeterminate type (mesial fragments); 2, 4-5: points with tongue-shaped base; 6: double beveled point on complete antler segment; 7: single beveled point (CAD JMP). 


\begin{tabular}{|l|l|c|c|c|}
\hline \multicolumn{2}{|l|}{} & Inférieur & Supérieur & Total \\
\hline \multirow{2}{*}{ Déchets de débitage } & d. DR longitudinal & 10 & 4 & $\mathbf{1 4}$ \\
\cline { 2 - 5 } & d. transversal par sciage & 0 & 1 & $\mathbf{1}$ \\
\hline Déchets de façonnage sur baguette & 1 & 1 & $\mathbf{2}$ \\
\hline Ébauches d'aiguille & 2 & 1 & $\mathbf{3}$ \\
\hline Objets finis sur baguette : aiguilles & 10 & 8 & $\mathbf{1 8}$ \\
\hline Objets finis sur hémi-côte : lissoirs & outils spatuliformes & 1 & 0 & $\mathbf{1}$ \\
\cline { 2 - 5 } & «pilons » & 1 & 0 & $\mathbf{1}$ \\
\cline { 2 - 5 } & poinçons & 0 & 1 & $\mathbf{1}$ \\
\cline { 2 - 5 } & retouchoirs & 0 & 6 & $\mathbf{6}$ \\
\hline \multirow{3}{*}{ Objets finis sur éclat } & outils spatuliformes & 2 & 0 & $\mathbf{2}$ \\
\cline { 2 - 5 } & outils intermédiaires & 0 & 1 & $\mathbf{1}$ \\
\hline \multirow{2}{*}{ Objets sur support indéterminé } & & 2 & 5 & $\mathbf{7}$ \\
\hline Indéterminés & & $\mathbf{3 2}$ & $\mathbf{2 8}$ & $\mathbf{6 0}$ \\
\hline
\end{tabular}

Tabl. 5 - SG (Trécolle) : composition techno-typologique de l'industrie en os par ensemble archéostratigraphique (d. : débitage; DR : double rainurage; sup. : support).

Table 5-SG (Trécolle) : Techno-typological composition of the bone industry (d: debitage; DR: groove and splinter technique; sup.: blank type).

baguette, large de $3 \mathrm{~mm}$, par double rainurage opposé sur ulna d'antilope saïga (fig. $24, \mathrm{n}^{\circ} 2$ ). Le seul indice d'extraction multiple que l'on peut peut-être, par sa patine, rapprocher de l'ensemble supérieur est un déchet sur métacarpien de Renne provenant de l'ensemble médian; il présente les traces du débitage de deux baguettes (une sur la face médiale et une sur la face latérale : fig. 24, $\mathrm{n}^{\circ} 3$ ). L'ensemble supérieur a également livré un possible fragment d'ébauche d'aiguille, un déchet de façonnage de baguette avec traces de sectionnement transversal par sciage, et huit fragments d'aiguilles. Comme dans l'ensemble inférieur, ces dernières pièces, longues de 8 à $26 \mathrm{~mm}$, sont de section ovale ou circulaire (calibre moyen : $2,5 \times 2,1 \mathrm{~mm}$ ) et seules deux ont conservé une portion de leur chas (fig. 24, $\mathrm{n}^{\text {os }} 4$ et 5).

Cet ensemble ne livre aucun lissoir sur côte, mais deux pièces - un déchet de sectionnement de côte de boviné par sciage transversal et un fragment de côte entièrement raclé - pourraient peut-être se rapporter à la fabrication de ce type d'objet. L'usage de retouchoirs sur éclat diaphysaire est attesté par six exemplaires (dont trois sur éclats d'os long d'ongulé de grande taille, deux sur éclat d'os long - fémur et tibia - d'antilope saïga et un sur éclat d'humérus de Boviné : fig. 24, nos 6 et 7). On compte également un court poinçon sur éclat diaphysaire de radius de renne et trois fragments d'os d'oiseau travaillés (deux fragments d'os long, dont un de la taille du harfang, avec des traces de raclage; un fragment d'ulna d'espèce indéterminée avec deux profondes incisions transversales).

Enfin, au sein du matériel découvert hors stratigraphie mais portant la patine de l'ensemble supérieur, il faut signaler la présence d'un fragment distal de bâton percé façonné sur cheville osseuse d'antilope saïga (fig. 24, $\mathrm{n}^{\circ} 8$ ). Il s'agit là, à notre connaissance, de l'unique cas au
Paléolithique supérieur où cet élément osseux a été choisi pour la fabrication d'un objet de ce type.

\section{Éléments de comparaison}

Dans l'ensemble inférieur comme dans l'ensemble supérieur, l'industrie en bois de cervidé est caractérisée par l'exploitation exclusive du bois de renne - plus exactement des bois de chute et de massacre de module moyen et gros, principalement utilisés pour la production de baguettes par double rainurage. Le faible effectif des séries et l'état de fragmentation du matériel rendent difficile l'appréciation de la productivité des débitages, mais on peut souligner que, dans les deux ensembles, des extractions uniques comme des extractions multiples (deux ou trois baguettes par bloc) sont attestées. L'équipement en bois de cervidé de l'ensemble supérieur est moins diversifié que celui de l'ensemble inférieur, avec la disparition des alênes et des outils mousses sur épois. Les deux principaux produits sur baguette - outils intermédiaires et pointes de projectile - présentent des caractères différents : ceux de l'ensemble supérieur sont nettement plus larges, attestant d'un changement dans le calibre des baguettes produites; ils sont parfois décorés, ce qui n'est jamais le cas dans l'ensemble inférieur. Les armatures de l'ensemble supérieur sont également plus longues que leurs homologues de l'ensemble inférieur, portent beaucoup plus rarement des rainures longitudinales et présentent des types de base différents (base biseautée et base en languette $v s$ base pleine). Ces deux dernières différences signalent manifestement une évolution dans les modalités de montage des armatures osseuses (mode d'emmanchement sur la hampe, combinaison avec les armatures microlithiques). 

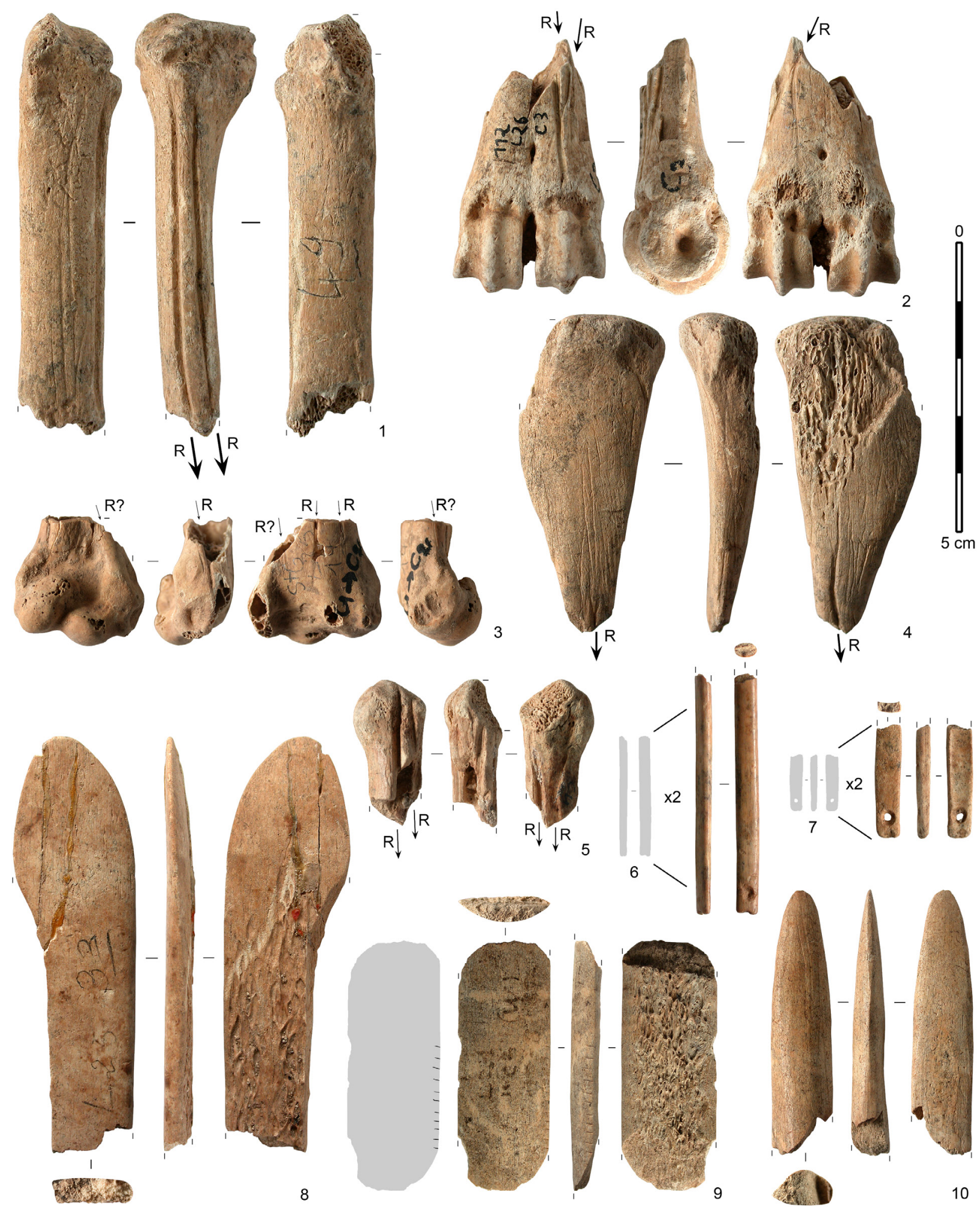

Fig. 23 - SG (ensemble inférieur) : industrie en os. 1-5 : déchets d'extraction de baguettes par double rainurage longitudinal (1 : sur métatarsien d'ours; $2:$ sur métacarpien d'antilope saïga; $3:$ sur humérus d'oie; $4:$ sur métatarsien de cheval; $5:$ sur métacarpien de loup; R : rainure); 6-7 : fragments mésio-proximaux d'aiguille à chas avec traces de réfection du chas 8-9: fragments de lissoir sur hémi-côte ( 8 : fragment mésio-distal; 9 : fragment mésial avec série d'incisions sur le bord et croquis montrant l'emplacement des incisions); 10 : fragment mésio-distal d'outil spatuliforme (DAO JMP).

Fig. 23 - SG (lower stratigraphic unit): bone industry. 1-5: waste products of splinter production with the groove and splinter technique (1: on a bear metatarsal; 2: on a saiga antelope metacarpal; 3: on a goose humerus; 4: on a horse metatarsal; 5: on a wolf metacarpal; R: groove); 6-7: mesio-proximal fragments of needle with traces showing repair of the eye; 8-9: smoother fragments on splinter taken from one side of a rib (8: mesio-distal fragment; 9: mesial fragment with a series of incisions along the edge and sketch showing the placement of these incisions); 10: mesio-distal fragment of a spatula-shaped tool (CAD JMP). 

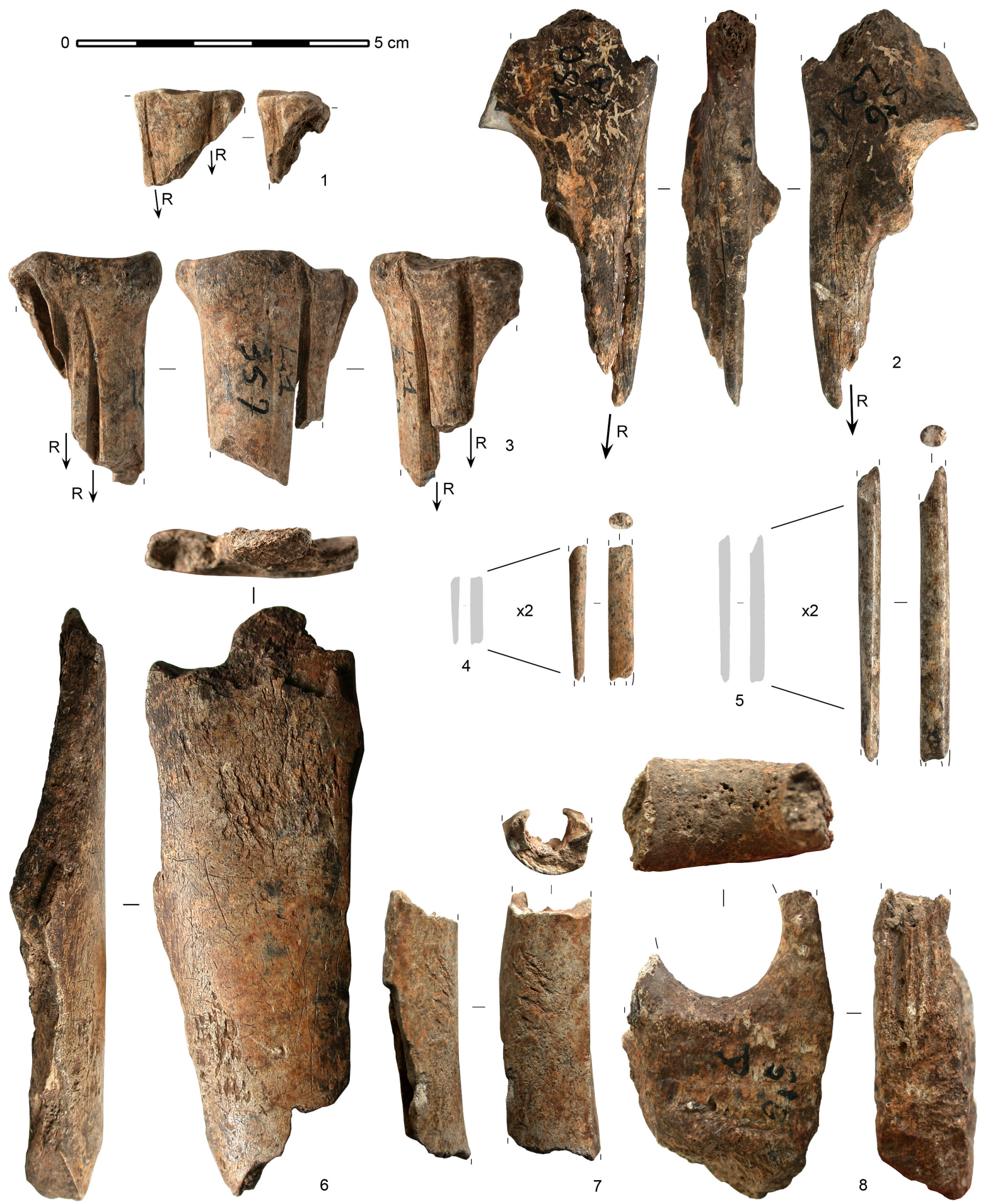

Fig. 24 - SG (ensemble supérieur) : industrie en os. 1-3 : déchets d'extraction de baguettes par double rainurage longitudinal (1 : sur métacarpien d'antilope saïga; 2 : sur ulna d'antilope saïga; 3 : sur métacarpien de renne; $\mathrm{R}:$ rainure); $4-5:$ fragments mésiaux d'aiguille à chas, avec une portion du chas préservée; $6:$ retouchoir sur éclat d'os long de grand ongulé, avec deux plages d'utilisation; 7 : fragment de retouchoir sur éclat de fémur d'antilope saïga; 8 : fragment de bâton percé sur cheville osseuse d'antilope saïga. La pièce $\mathrm{n}^{\circ} 3$ provient de l'ensemble médian et la pièce $\mathrm{n}^{\circ} 8$ a été trouvée hors stratigraphie mais les deux présentent la patine des pièces de l'ensemble supérieur (DAO JMP).

Fig. 24 - SG (upper stratigraphic unit): bone industry. 1-3: waste products of splinter production with the groove and splinter technique (1: on a saiga antelope metacarpal; 2: on a saiga antelope ulna; 3: on a reindeer metacarpal; $R$ : groove); 4-5: mesial fragments of needles, with part of the eye intact; 6: rretoucher made on a flake of a long bone from a large ungulate, bearing two areas of use; 7: fragment of a retoucher made on a flake from the femur of a saiga antelope; 8: fragment of a perforated baton made on the horn core of a saiga antelope. Artefact no. 3 comes from the middle part of the stratigraphy and artefact no. 8 was found out of stratigraphic context, but both show the patina of pieces found in the upper stratigraphic unit (CAD JMP). 
Dans l'industrie en os, les principaux choix techniques paraissent similaires dans les deux ensembles : exploitation de métapodiens d'ongulés et d'os longs d'oiseaux par rainurage longitudinal pour la production d'aiguilles à chas. Tout au plus le choix des blocs semblet-il plus diversifié dans l'ensemble inférieur, avec notamment l'exploitation d'os de carnivores (ours et loup). La productivité des débitages est, là encore, variable dans les deux ensembles (présence d'extractions uniques et multiples) et son estimation ne repose que sur un petit nombre de pièces. Soulignons également la présence d'un outillage non façonné ( « pilon », retouchoirs) ou peu façonné (outil spatuliforme, poinçon), attesté surtout dans l'ensemble supérieur.

L'industrie osseuse de l'ensemble inférieur peut être rapprochée de plusieurs séries attribuées au Magdalénien inférieur (Gandil c. 20 et 23-25 : Langlais et al., 2007; les Scilles : Langlais et al., 2010; le Taillis des Coteaux AG-IIIa : Primault et al., 2010 ; le Petit Cloup Barrat c. 4 : Ducasse et al., 2011); des séries mélangées ayant livré entre autres des éléments diagnostiques ou des dates ${ }^{14} \mathrm{C}$ du Magdalénien inférieur (Lassac, fouilles Barthès et ramassages Bennes : Pétillon et Ducasse, 2012; les Harpons, couches C et D : Ducasse et Renard, 2013; Reverdit, fouilles Delage : Bourdier et al., 2014a). Elle peut également être comparée à des séries pour lesquelles un rapprochement avec le Magdalénien inférieur peut être suggéré sur des bases typologiques (Gabillou : Gaussen, 1964; Lascaux : Leroy-Prost, 2008). Du point de vue technologique, toutes sont dominées par la production d'objets sur baguettes extraites par double rainurage, tant sur os que sur bois de cervidé. Dans les cas où la productivité des débitages a pu être évaluée, elle semble être globalement assez faible (peu de supports extraits de chaque bloc, même si quelques cas d'extractions multiples sont attestés). Dans plusieurs de ces séries, la typologie de l'équipement osseux présente des analogies avec l'ensemble inférieur des fouilles Trécolle. Les outils mousses sur épois sont ainsi attestés à Gandil (collection Chaillot : Pétillon, observation personnelle), à Reverdit (Bourdier et al., 2014a, fig. 10), à Lassac (fouilles Bennes : Pétillon, observation personnelle) et peut-être à Gabillou (Gaussen, 1964, fig. 8, no 3). Les alênes en bois de cervidé sont présentes à Gandil, c. 23 (Langlais et al., 2007, fig. 11), à Reverdit (Bourdier et al., 2014a, fig. 11) et dans plusieurs couches de la grotte des Harpons (avec des mélanges manifestes entre couches ${ }^{(3)}$ : voir par exemple Pétillon, 2006, fig. 1). En ce qui concerne la typologie des armatures osseuses, la plupart de ces séries sont, comme celle de l'ensemble inférieur des fouilles Trécolle, marquées par la présence quasi exclusive de pointes à base pleine : c'est le cas à Gandil, c. 20 et 23-25 (hormis une pointe à méplat mésial), aux Scilles (hormis deux pointes à biseau simple, dont le degré d'association avec le reste du matériel demanderait sans doute à être rediscuté), à Reverdit (hormis, là encore, une pointe à méplat mésial), à Lascaux et à Gabillou (au Taillis des Coteaux EG-III et AG-III et au Petit Cloup Barrat, c. 4, le mode d'emmanchement des pointes reste indéterminé mais, en tout cas, aucune pointe à base biseautée n'a été identifiée). Les armatures osseuses de Gabillou (Gaussen, 1964, fig. 8, $\mathrm{n}^{\text {os }}$ 6-11) présentent une nette ressemblance morphométrique avec certaines pointes de l'ensemble inférieur des fouilles Trécolle et constituent le parallèle le plus proche que nous ayons pu trouver pour ces pièces.

Sur le plan technologique, l'industrie osseuse de l'ensemble supérieur s'inscrit globalement dans la variabilité du Magdalénien moyen; les quelques particularités - notamment l'utilisation importante de l'antilope saïga dans l'industrie en os - s'expliquent par la nature des ressources localement disponibles, tandis que le faible effectif de la série et sa conservation médiocre empêchent une analyse technologique plus fine. Sur le plan de la typologie, plus particulièrement celle des armatures de projectile, il faut souligner l'absence à SG de marqueurs habituels du Magdalénien moyen telles les pointes de Lussac-Angles, les pointes à biseau double du « Magdalénien à navettes » (Allain et al., 1985) ou les baguettes demi-rondes. On y trouve en revanche une série d'armatures particulières - larges et plates, à base en languette, parfois décorées de motifs géométriques - pour lesquelles nous disposons actuellement de peu de points de comparaison. Ces armatures sont absentes du Magdalénien moyen pyrénéen, et les quelques pièces analogues que nous avons pu observer renvoient plutôt à des gisements situés à l'est de SG : des pointes très similaires se trouvent en effet à Gandil (collection Chaillot) et peutêtre à Combe-Cullier (Pétillon, observation personnelle).

\section{LES VESTIGES FAUNIQUES}

Un abondant matériel osseux comprenant près de 30000 restes a été étudié. La détermination a été effectuée à l'aide de la collection de comparaison du laboratoire PACEA, des travaux de référence portant sur les Vulpinés (Poplin, 1976a; Altuna, 2004), les Léporidés (Morel et Müller, 1997) et les Canidés (Boudadi-Maligne, 2010), ainsi que des données inédites de l'un d'entre nous (JBM) pour les Mustélidés. En ce qui concerne la grande faune, les pièces inférieures à $2 \mathrm{~cm}$ non déterminables ont été triées par grande catégorie osseuse : bois, crâne, dents, vertèbres, côtes, fragment de diaphyse d'os long, spongieux, tissu compact, en notant la présence ou non de traces de combustion. Que ce soit pour le NMI ou le NME, les nombres minimums de combinaison ont été calculés en prenant en compte l'âge et la taille. Pour les oiseaux, le NMI est de fréquence.

Les observations taphonomiques ont été menées afin de reconstituer les grandes lignes de l'histoire de l'accumulation osseuse. Elles ont été réalisées sous lumière rasante avec une loupe manuelle $(\times 10)$ pour les restes de grands mammifères de plus de $2 \mathrm{~cm}$ et sous stéréomicroscope $(\times 10$ à $\times 40)$ pour les mésomammifères et les restes aviaires déterminés au-delà de cette classe. Pour la reconnaissance des traces, nous avons retenu les critères classiquement utilisés en taphonomie (White, 1992; Lyman, 


\begin{tabular}{|l|c|c|} 
Traces & Inférieur & Supérieur \\
\hline Anthropiques & $28,1(1572)$ & $30,7(1669)$ \\
\hline Carnivores & $0,8(46)$ & $0,8(39)$ \\
\hline Racines & $2,8(167)$ & $6,7(378)$ \\
\hline Piétinement & $1,8(106)$ & $0,9(50)$ \\
\hline Fissures longitudinales & $11,9(702)$ & $8,4(476)$ \\
\hline Desquamation & $0,4(23)$ & $2,2(124)$ \\
\hline Dissolution & $20,6(1214)$ & $24,1(1362)$ \\
\hline Concrétion & $0,5(29)$ & $9,6(544)$ \\
\hline Abrasion & $0,2(15)$ & $0,5(29)$ \\
\hline
\end{tabular}

Tabl. 6 - SG (Trécolle) : répartition archéostratigraphique des types de traces observées sur les ossements de grands mammifères (en $\%$ et NR).

Table 6-SG (Trécolle) : Distribution, by unit, of taphonomic marks observed on bones of large mammals (in \% and NISP).

1994; Fischer, 1995; Blumenschine et al., 1996). Pour l'interprétation des traces de boucherie, différents référentiels actualistes ont été pris en compte (Binford 1981; Laroulandie, 2000; Nilssen 2000; Lloveras et al., 2009; Mallye, 2011; Val et Mallye, 2011; Costamagno, 2012).

Les restes de grands mammifères ont fait l'objet d'une première publication (Costamagno, 2001). Ils sont ici présentés sur la base de la réévaluation archéostratigraphique et à la lumière de nouveaux cadres interprétatifs, expliquant certaines disparités avec les interprétations de l'époque, en particulier concernant le transport des carcasses.

\section{Étude taphonomique}

Sur les quelque 28000 restes de grands mammifères décomptés, près de 12000 ont fait l'objet d'observations taphonomiques. Dans les deux ensembles, le matériel osseux est relativement bien conservé. De petites cupules de dissolution sont présentes sur près d'un quart des ossements (tabl. 6). C'est l'attaque taphonomique la plus fréquente à laquelle viennent s'ajouter, en particulier dans l'ensemble supérieur, des dépôts concrétionnés et des traces de racines. L'intensité de ces attaques est néanmoins très faible puisque moins de $5 \%$ des ossements présentent des surfaces mal conservées. Un nombre non négligeable de pièces présentent des fissures longitudinales qui pourraient indiquer un enfouissement relativement lent des vestiges (Andrews et Cook, 1985; Fosse et al., 2004; Mallye et al., 2009). La météorisation n'a cependant joué qu'un rôle mineur dans la conservation différentielle des ensembles osseux, très peu de vestiges présentant les stades ultimes de weathering (Behrensmeyer, 1978). Les carnivores n'ont eu aussi qu'un faible impact sur les restes fauniques. Quelques traces de dents, principalement des pits, attestent d'un charognage peu intense des restes de carcasses abandonnés par les hommes. En effet, la fréquence des traces de boucherie désigne sans conteste l'homme comme le principal accumulateur des restes d'Ongulés. L'importante fragmentation du matériel ${ }^{(4)}$ est principalement imputable aux activités humaines; en témoignent les nombreuses traces de percussion et les morphologies des bords de fracture des diaphyses d'os longs qui sont typiques d'une fracturation sur os frais (Villa et Mahieu, 1991).

À côté de ces agents taphonomiques « classiques », la « taphonomie de laboratoire » a fortement biaisé l'échantillon osseux étudié (Costamagno, 1999). Les pertes de matériel mises en évidence, en lien avec de mauvaises conditions de stockage, ont engendré une sous-représentation des fragments de diaphyse d'os longs et plus largement des restes de petite taille. Le pourcentage de pièces inférieures à $2 \mathrm{~cm}$ et le pourcentage de fragments de diaphyse relativement aux restes dentaires montre néanmoins que certains carrés n'ont pas subi de perte. C'est le cas du carré M21 pour l'ensemble supérieur et des carrés L21 et L22 pour l'ensemble inférieur (tabl. 7). Pour les profils squelettiques, les fragments diaphysaires étant généralement mieux représentés que les extrémités articulaires (Marean et Frey, 1997; Bartram et Marean, 1999), les biais taphonomiques peuvent être importants mais les analyses réalisées ont montré que ce n'était pas le cas ici (Costamagno, 2002).

Parmi les cinq ossements ${ }^{(5)}$ de Canidés rapportés au loup (tabl. 8), un seul porte des traces d'activité

\begin{tabular}{|c|c|c|c|c|c|c|c|c|c|c|}
\hline & \multicolumn{7}{|c|}{ Inférieur } & \multicolumn{3}{|c|}{ Supérieur } \\
\hline & L21 & L22 & L23 & L24 & L25 & L26 & L28 & L21 & L22 & M21 \\
\hline $\mathrm{NR}<2 \mathrm{~cm}$ & 6842 & 4081 & 0 & 32 & 0 & 0 & 0 & 286 & 15 & 4375 \\
\hline$\%<2 \mathrm{~cm}$ & 70,1 & 67,2 & 0 & 15 & 0 & 0 & 0 & 4,2 & 2,2 & 87,2 \\
\hline NR dents & 150 & 83 & 25 & 62 & 40 & 45 & 19 & 210 & 179 & 65 \\
\hline NR diaphyses & 1456 & 969 & 1 & 5 & 2 & 5 & 2 & 709 & 26 & 1382 \\
\hline \% diaphyses & 90,7 & 92,1 & 3,8 & 7,5 & 4,8 & 10,0 & 9,5 & 77,1 & 12,7 & 95,5 \\
\hline
\end{tabular}

Tabl. 7 - SG (Trécolle) : répartition archéostratigraphique des pièces inférieures à $2 \mathrm{~cm}$ et des fragments diaphysaires (en \% et NR; $\%$ diaphyses $=$ NR diaphyses $/($ NR dents + NR diaphyses $) \times 100)$.

Table 7-SG (Trécolle): Distribution, by unit, of artefacts smaller than $2 \mathrm{~cm}$ and of diaphysis fragments (in \% and NISP; \% diaphysis $=$ NISP diaphysis / (NISP teeth + NISP diaphysis $) \times 100)$. 


\begin{tabular}{|c|c|c|c|c|c|c|c|c|}
\hline \multirow[b]{2}{*}{ Taxon } & \multicolumn{4}{|c|}{ Inférieur } & \multicolumn{4}{|c|}{ Supérieur } \\
\hline & NRD & $\%$ NRD & NMI & $\% \mathrm{NMI}$ & NRD & $\% \mathrm{NRD}$ & NMI & $\% \mathrm{NMI}$ \\
\hline Ursidae & 1 & 0,0 & 1 & 1,4 & 0 & - & - & - \\
\hline Loup (Canis lupus) & 4 & 0,1 & 2 & 2,9 & 1 & 0,0 & 1 & 1,4 \\
\hline Vulpinae & 14 & 0,5 & 1 & 1,4 & 0 & - & - & - \\
\hline Renard (Vulpes vulpes) & 0 & - & - & - & 6 & 0,2 & 1 & 1,4 \\
\hline Belette (Mustela nivalis) & 1 & 0,0 & 1 & - & 0 & - & - & - \\
\hline Mustela sp. & 0 & - & - & - & 1 & 0,0 & 1 & 1,4 \\
\hline Lynx (Lynx lynx) & 1 & 0,0 & 1 & - & 0 & - & - & - \\
\hline Bovinae & 117 & 4,0 & 3 & 4,3 & 66 & 2,3 & 2 & 2,7 \\
\hline Antilope saiga (Saiga tatarica) & 1438 & 48,7 & 18 & 25,7 & 2067 & 73,5 & 43 & 58,1 \\
\hline Renne (Rangifer tarandus) & 498 & 16,9 & 5 & 7,1 & 367 & 13,1 & 5 & 6,8 \\
\hline Cerf (Cervus elaphus) & 2 & 0,1 & 1 & 1,4 & 1 & 0,0 & 1 & 1,4 \\
\hline Cheval (Equus caballus) & 183 & 6,2 & 6 & 8,6 & 141 & 5,0 & 5 & 6,8 \\
\hline Equus hydruntinus & 1 & 0,0 & 1 & 1,4 & 16 & 0,6 & 1 & 1,4 \\
\hline Lièvre (Lepus cf. timidus) & 4 & 0,1 & 1 & 1,4 & 2 & 0,1 & 1 & 1,4 \\
\hline Spermophile (Citellus sp.) & 29 & 1,0 & 4 & 5,7 & 6,0 & 0,2 & 3 & 4,1 \\
\hline NRD Mammifères & 2293 & 77,7 & 45 & 64,3 & 2674 & 95,1 & 64 & 86,5 \\
\hline Anser sp. & 1 & 0,0 & 1 & 1,4 & 0 & 0,0 & - & - \\
\hline Lagopus sp. & 25 & 0,8 & 2 & 2,9 & 3 & 0,1 & 1 & 1,4 \\
\hline Chouette harfang (Bubo scandiacus) & 633 & 21,4 & 22 & 31,4 & 127 & 4,5 & 6 & 8,1 \\
\hline Grand corbeau (Corvus corax) & - & - & - & - & 5 & 0,2 & 2 & 2,7 \\
\hline Passériformes (petits) & - & - & - & - & 2 & 0,1 & 1 & 1,4 \\
\hline NRD Oiseaux & 659 & 22,3 & 25 & 35,7 & 137 & 4,9 & 10 & 13,5 \\
\hline NRD total & 2952 & 100,0 & 70 & 100,0 & 2811 & 100,0 & 74 & 100,0 \\
\hline Indice de Simpson total & 3,120 & & & & 1,747 & & & \\
\hline Indice de Simpson Ongulés & 2,150 & & & & 1,582 & & & \\
\hline Carnivore indéterminé & - & & & & 1 & & & \\
\hline Ongulé de petite taille & 8 & & & & 9 & & & \\
\hline Ongulé de petite ou moyenne taille & 1890 & & & & 1499 & & & \\
\hline Ongulé de taille moyenne & 29 & & & & 3 & & & \\
\hline Ongulé de taille moyenne ou grande & 1027 & & & & 553 & & & \\
\hline Mammifère indéterminé & 851 & & & & 1028 & & & \\
\hline Oiseau indéterminé & 1696 & & & & 203 & & & \\
\hline Total & 8453 & & & & 6107 & & & \\
\hline
\end{tabular}

Tabl. 8 - SG (Trécolle) : répartition archéostratigraphique de l'abondance relative en nombre de restes (NRD) et en nombre minimum d'individus (NMI) des taxons identifiés.

Table 8 -SG (Trécolle): Distribution, by unit, of the relative abundance of identified taxa, expressed in number of identified specimens (NISP) and minimum number of individuals (MNI).

humaine : comme vu précédemment, il s'agit d'un cinquième métacarpien droit (fragment proximal) provenant de l'ensemble inférieur, utilisé comme matière première.

Les surfaces osseuses des restes de mésomammifères et d'oiseaux sont également bien conservées dans leur ensemble et se prêtent favorablement à la lecture des traces de prédation. Sur les vestiges de mésomammifères, des traces anthropogènes ont été observées sur le renard et le lièvre des deux ensembles considérés. Plusieurs restes de spermophile (NISP $=5 / 29$ dans l'ensemble inférieur;
3/6 dans l'ensemble supérieur) montrent des altérations résultant de la digestion qui indiquent un apport par un prédateur non humain dont l'identité demeure inconnue (voir ci-dessous fig. 28e). La belette, le lynx et les putois ne révèlent quand à eux aucun indice permettant de statuer sur les causes de leur présence dans le gisement. Sur les restes aviaires, les traces de prédation se résument à des marques d'activité humaine (NISP $=131 / 796)$ et s'observent sur tous les taxons identifiés à l'exception des petits passériformes. Elles indiquent que les hommes du 
Magdalénien inférieur et moyen ont pratiqué la chasse aux oiseaux. Tous les os longs de harfang sont intensément fragmentés et les nombreuses esquilles rapportées aux oiseaux (tabl. 8) ont, pour la majorité d'entre elles, des tailles et des circonférences compatibles avec ce rapace. Bien qu'une étude précise reste à mener, la présence de nombreux tronçons centimétriques de radius cassés transversalement indique d'ores et déjà une fragmentation post-dépositionnelle importante.

La gamme des espèces animales exploitées par les chasseurs-cueilleurs comprend donc des gibiers à poils, de petite, moyenne et grande taille ainsi que des gibiers à plumes. Notons que le spermophile, pourtant présent dans l'environnement, ne semble pas avoir intéressé les hommes du Magdalénien inférieur et moyen, contrairement à ce qui fut le cas lors de la phase récente (e. $g$. Langlais et al., 2012 et 2014).

\section{Proies chassées et saison d'abattage}

Les cortèges fauniques sont comparables sur l'ensemble de la séquence stratigraphique mais la part des principaux taxons évolue. Dans l'ensemble inférieur, en nombre de restes, l'antilope saïga est la proie principale des Magdaléniens suivie du harfang, du renne ${ }^{(6)}$, du cheval puis des Bovinés (tabl. 8). En NMI, le saïga est surpassé par le harfang et sa part diminue au profit des taxons peu représentés (Poplin, 1976b). Le cheval devient le deuxième

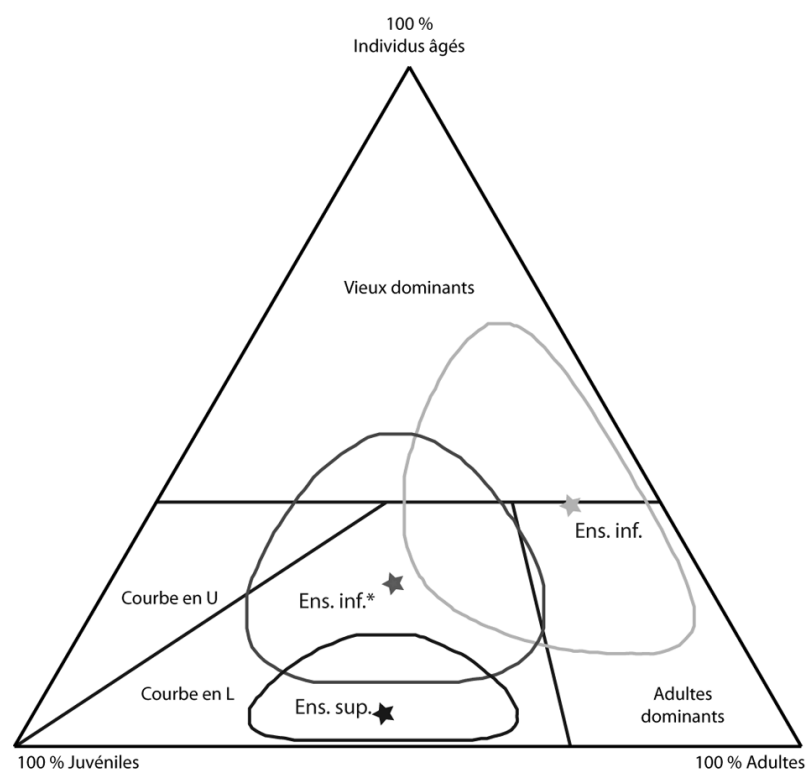

Fig. 25 - SG (Trécolle) : structure d'âge des antilopes saïga sur un diagramme ternaire corrigé in Costamagno, 1999 avec les ellipses de confiance à $95 \%$ (Steele et Weaver, 2002). Ens. inf.* correspond à la structure d'âge tenant compte des restes dentaires et des ossements.

Fig. 25 - SG (Trécolle): mortality profile of saiga antelope plotted on a corrected triangular diagram in Costamagno 1999. The ellipse represents the 95\% confidence interval (Steele and Weaver, 2002). Ens. Inf.* represents the age structure profile which takes into account dental and osseous remains.
Ongulé le plus chassé. Au Magdalénien moyen, la part des oiseaux diminue fortement. Les quatre Ongulés précédemment cités sont toujours chassés mais l'antilope saïga tient une place plus importante comme l'atteste l'indice de $\operatorname{Simpson}^{(7)}$ qui témoigne d'un spectre faunique moins diversifié, que l'ensemble du spectre soit pris en compte ou seulement les Ongulés (tabl. 8). Au vu de l'épaisseur des ensembles, les assemblages osseux sont de toute évidence le reflet de chasses successives. Dans l'ensemble inférieur, au moins soixante-deux animaux dont vingt-deux harfangs, dix-huit antilopes saïga et six chevaux ont été exploités; dans l'ensemble supérieur, il s'agit d'un minimum de soixante-neuf individus dont quarante-trois saïgas, six harfangs, cinq chevaux et rennes. Du fait de la faible surface fouillée ${ }^{(8)}$, on peut supposer que chaque ensemble devait contenir plusieurs centaines de carcasses.

Seule l'antilope saïga permet l'étude des profils de mortalité ${ }^{(9)}$. Pour les autres espèces, ce sont majoritairement des individus adultes qui ont été abattus. Concernant les chevaux, la présence de quelques immatures et la rareté des canines semblent indiquer des chasses menées préférentiellement sur des groupes familiaux comme cela est souvent le cas au Paléolithique. Dans l'ensemble inférieur, les restes dentaires de saïga témoignent de chasses menées sur des adultes dans la force de l'âge mais surtout sur de vieux individus de plus de 7 ans (fig. 25). La prise en compte des restes osseux modifie cependant la courbe d'abattage dont le profil devient alors caractéristique d'une courbe en $\mathrm{L}^{(10)}(i$. e. Stiner, 1990). Les mâles dépassant rarement l'âge de 5 ans (Bannikov, 1967), l'abondance de ces vieux animaux pourrait indiquer des chasses menées sur des groupes de femelles, l'absence de cheville osseuse confirmant cette hypothèse. La saison d'abattage est difficile à appréhender : sur les dix dents étudiées pour la cémentochronologie, une seule est suffisamment bien préservée. L'individu aurait été abattu durant la bonne saison ${ }^{(11)}$. Dans l'ensemble supérieur, la présence de chevilles osseuses montre que des mâles ont été chassés à côté de femelles et de jeunes. Le profil de mortalité est aussi typique d'une courbe en L mais, relativement à l'ensemble inférieur, il comporte plus de jeunes et moins de vieux individus. Ici encore, les résultats de l'étude cémentochronologique sont décevants puisque sur les trente-sept individus, seules six lames sont lisibles. Toutes indiquent des animaux abattus durant la bonne saison. D'après les restes dentaires, aucun immature n'a moins de deux mois; les saïgas mettant bas en avril-mai, les chasses auraient pu se dérouler durant tout l'été. Le bois de massacre de renne mâle mis au jour dans cet ensemble atteste aussi d'une chasse durant la bonne saison.

Pour les oiseaux, les arguments directs de bonne saison que constituent l'os médullaire et l'os immature (Serjeantson, 2009) manquent. Or, l'absence d'os médullaire n'est pas à mettre en relation avec celle des femelles puisque les mesures effectuées sur certains ossements (selon la méthode in Chauviré, 1965 améliorée in Gourichon, 1994) montrent qu'au moins huit femelles adultes 
ont été capturées dans l'ensemble inférieur. Actuellement, l'os médullaire serait présent au sein des populations de Harfang entre mi-avril et juin (Laroulandie, 2000, p. 110). Bien que reposant sur des arguments négatifs, une capture entre juillet et février est probable, ce qui englobe largement la période estimée de la chasse aux saïgas.

\section{Exploitation des ongulés}

\section{Transport des carcasses}

La bonne conservation des ossements et le faible impact des agents taphonomiques non humains sur les restes osseux sont des atouts pour l'étude de la représentation des éléments squelettiques. Mais la faible surface fouillée et la perte de matériel durant le stockage rendent délicate l'interprétation des profils squelettiques en terme de transport. Les restes dentaires étant largement surreprésentés (voir ci-dessus), pour les comparaisons avec les éléments post-crâniens, seuls les fragments mandibulaires ont été pris en compte. Les côtes, difficilement identifiables d'un point de vue taxinomique en raison de leur forte fragmentation, ont aussi été exclues pour le décompte des NME. Dans un premier temps, nous discutons de la représentation différentielle des squelettes de Saïga puis plus succinctement des trois autres gibiers principaux.

Dans l'ensemble inférieur, les vertèbres sont largement sous-représentées (fig. 26a). Pour les côtes, la quantification est plus délicate mais le nombre de fragments de côtes d'ongulés de taille petite ou moyenne reste faible $(\mathrm{NR}=988)$ relativement au nombre minimum de saïgas $^{(12)}$. Les os longs des membres sont abondants, en particulier le fémur et les métapodes. Les mandibules ont un pourcentage de survie de l'ordre de $40 \%$ tout comme les premières phalanges. Les phalanges 2 et 3 sont proches de $25 \%$. En prenant en compte les restes dentaires, dix-huit crânes et seize mandibules ont pu être dénombrés. Les carcasses d'antilope semblent donc incomplètes : une partie des côtes mais surtout les vertèbres pourraient avoir été abandonnées sur le site d'abattage ou être présentes dans un autre secteur de l'occupation. Les membres ont été introduits préférentiellement sur le site. La sousreprésentation des phalanges ne semble pas être liée à un biais taphonomique car les carpiens et les tarsiens, également de petite taille et de densité moindre, ont des pourcentages de survie comparables aux os longs. Pour les crânes, en raison de la perte de matériel, il est impossible de savoir s'ils ont été introduits dans les mêmes proportions que les membres. En revanche, crâne et mandibule semblent avoir été transportés conjointement.

Dans l'ensemble supérieur (fig. 26b), la représentation des éléments squelettiques est assez comparable à celle de l'ensemble inférieur. Le squelette axial post-crânien est rare ${ }^{(13)}$. Les phalanges sont présentes dans des proportions comparables et les carpiens-tarsiens sont relativement fréquents. Les os longs des membres sont les éléments squelettiques les plus abondants mais le tibia est nettement plus fréquent que le fémur. Contrairement à l'ensemble inférieur, les os coxaux, qui étaient très rares, sont présents dans des proportions comparables à la scapula ou au fémur. Au niveau des restes dentaires, trente-quatre crânes et cinquante-trois mandibules ont pu être dénombrés. Dans cet ensemble aussi, les squelettes d'antilopes sont donc incomplets. La sous-représentation du squelette axial semble encore plus accentuée que dans l'ensemble inférieur mais les biais engendrés par la taphonomie de laboratoire pourraient être en partie responsables de ces différences (tabl. 7). Les têtes ne sont pas complètes, les crânes étant largement sous-représentés, caractère qui

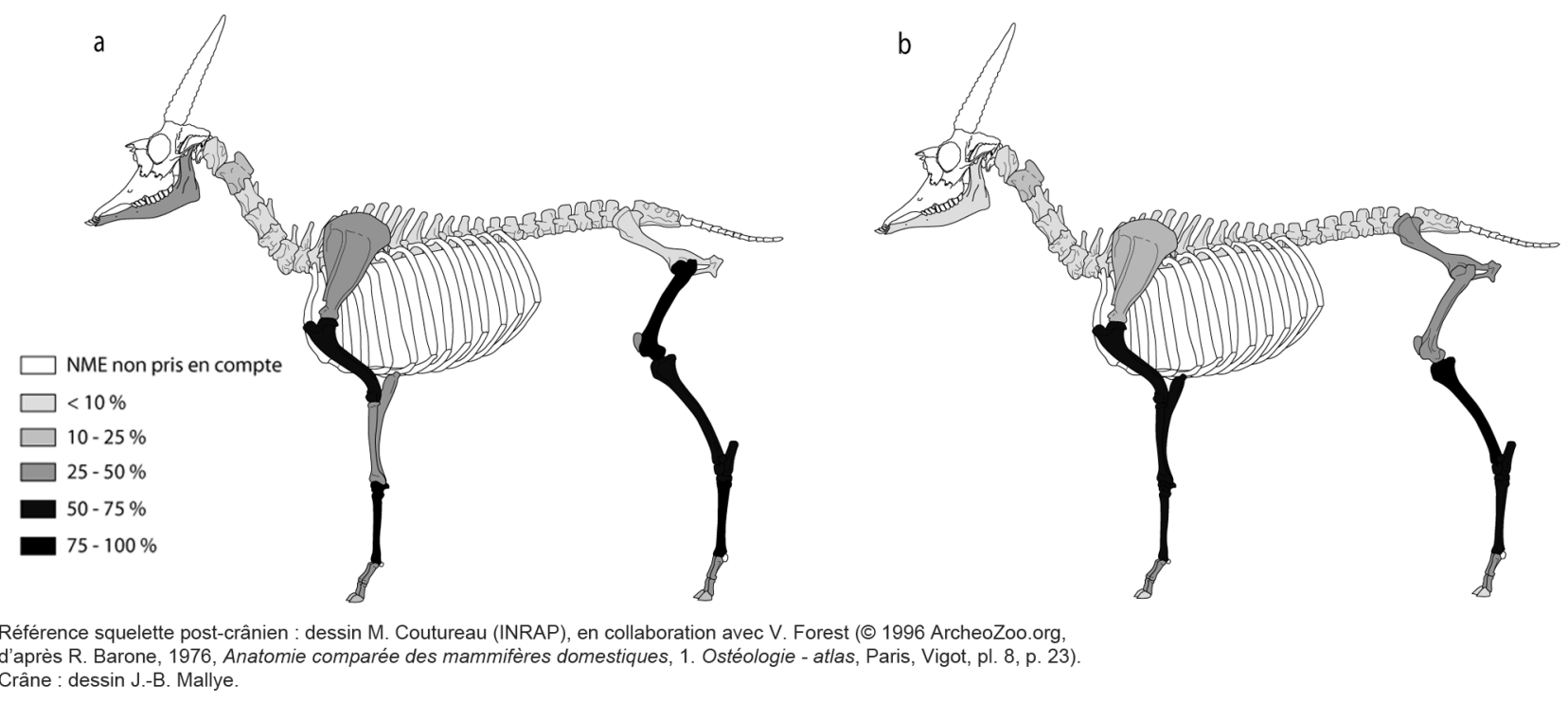

Fig. 26 - SG (Trécolle) : pourcentage de survie des éléments squelettiques d'Antilope saïga. a : ensemble inférieur; b : ensemble supérieur. Pour le décompte du pourcentage de survie des éléments crâniens, les restes dentaires ne sont pas pris en compte (DAO SC).

Fig. 26 - SG (Trécolle):percentage survival of skeletal elements of saiga antelope. a: lower stratigraphic unit; b: upper stratigraphic unit. For the breakdown of the percentage survival of cranial elements, dental remains have not been taken into account (CAD SC). 
distingue cet ensemble de l'ensemble sous-jacent. Du fait de la faible surface fouillée, il est difficile de trancher entre transport préférentiel des mandibules et biais spatial. Les membres ont probablement été introduits complets sur le site et les différences observées entre les os longs pourraient être dues à la répartition spatiale des vestiges et à la faible étendue de la fouille. Comme pour l'ensemble inférieur, la sous-représentation des phalanges pourrait avoir un lien avec les activités de boucherie.

En ce qui concerne les autres espèces, dans les deux ensembles, le renne présente globalement le même schéma que l'antilope saïga. Les têtes pourraient néanmoins avoir été plus fréquemment abandonnées sur le site d'abattage, tout comme les métapodes qui eux pourraient également avoir été utilisés ailleurs comme matrice de baguettes (tabl. 9). Les nombreux fragments de bois ${ }^{(14)}$ présents dans les deux niveaux ont donc probablement été introduits de façon autonome, ce que confirme l'étude de l'industrie osseuse. Dans l'ensemble inférieur, pour les gros ongulés, il semble y avoir un choix préférentiel en faveur des os longs les plus charnus (humérus et fémur) alors que, dans l'ensemble supérieur, la répartition des os longs est plus équilibrée, pouvant témoigner d'une introduction de membres entiers. Dans les deux niveaux, les phalanges sont nettement sous-représentées.

\section{Ressources recherchées}

Les traces de boucherie sont très abondantes (tabl. 10), ce qui témoigne d'une exploitation intensive des carcasses d'ongulés depuis le prélèvement de la peau jusqu'à l'extraction de la moelle.

\section{L'antilope saïga}

Les marques de boucherie observées sur les restes de saïga témoignent de pratiques bouchères relativement similaires entre les deux ensembles. Ces similarités ne doivent néanmoins pas masquer quelques différences.

Le prélèvement de la peau est attesté sur une quarantaine de restes de l'ensemble inférieur et plus d'une centaine de l'ensemble supérieur. Les stries longitudinales observées sur la branche horizontale des mandibules et sur les maxillaires témoignent du dépouillement de la tête (fig. 27). Les stries de dépouillement les plus fréquemment rencontrées correspondent au détachement de la peau de l'os. Sur les membres, ces traces sont principalement présentes sur les métapodes et les phalanges. La peau semble avoir été incisée très bas car très peu de métapodes portent des traces relatives à une incision circulaire. Les incisions longitudinales sont peu fréquentes, contrairement à ce qui a été produit expérimentalement

\begin{tabular}{|l|c|c|c|c|c|c|c|c|}
\multicolumn{1}{l}{} & \multicolumn{9}{c}{ Inférieur } & \multicolumn{4}{c|}{ Supérieur } \\
\cline { 2 - 10 } \multicolumn{1}{l|}{} & Saïga & Renne & Cheval & Bovinés & Saïga & Renne & Cheval & Bovinés \\
\hline Dents supérieures & 20 & 3 & 5 & 2 & 34 & 4 & 3 & 1 \\
\hline Dents inférieures & 16 & 5 & 6 & 3 & 53 & 5 & 5 & 2 \\
\hline Crâne & - & 1 & 2 & 2 & - & 1 & 1 & - \\
\hline Mandibule & 5 & - & 1 & 2 & 7 & 1 & 2 & - \\
\hline Vertèbres cervicales & 3 & - & 1 & 1 & 4 & - & 3 & 1 \\
\hline Vertèbres thoraciques & 1 & 1 & - & - & 1 & 5 & - & 1 \\
\hline Vertèbres lombaires & 1 & - & - & 1 & 1 & 1 & 1 & 1 \\
\hline Scapula & 6 & 2 & - & 2 & 18 & 2 & 1 & - \\
\hline Humérus & 12 & 1 & 6 & 3 & 18 & 5 & 2 & 1 \\
\hline Radius & 9 & 3 & 2 & 1 & 18 & 2 & 2 & 1 \\
\hline Carpiens & 13 & 2 & - & 1 & 17 & 1 & 1 & 1 \\
\hline Métacarpien & 14 & 3 & 1 & 1 & 22 & 4 & 2 & 1 \\
\hline Coxal & - & 1 & 1 & - & 12 & - & - & - \\
\hline Fémur & 14 & 3 & 3 & 2 & 11 & 3 & 1 & 1 \\
\hline Tibia & 8 & 2 & 2 & 1 & 29 & 4 & 1 & 1 \\
\hline Tarsiens & 10 & 2 & 2 & 1 & 19 & 1 & 1 & 1 \\
\hline Métatarsien & 13 & 2 & 1 & 1 & 21 & 2 & 1 & 1 \\
\hline Phalange 1 & 6 & 1 & 1 & 1 & 13 & 2 & 1 & - \\
\hline Phalange 2 & 4 & 1 & 1 & - & 10 & 2 & - & - \\
\hline Phalange 3 & - & 1 & - & 7 & 1 & - & - \\
\hline
\end{tabular}

Tabl. 9 - SG (Trécolle) : répartition archéostratigraphique du nombre minimum d'individus représenté selon l'élément squelettique considéré pour l'antilope saïga, le renne, le cheval et les Bovinés.

Table 9 - SG (Trécolle): distribution, by unit, of the minimum number of individuals represented, based on the skeletal element considered for saiga antelope, reindeer, horse and large bovids. 


\begin{tabular}{|c|c|c|c|}
\hline & $\begin{array}{c}\% \text { stries } \\
\text { transversales } \\
\text { ou obliques }\end{array}$ & $\begin{array}{c}\% \text { stries } \\
\text { longitudinales }\end{array}$ & $\%$ percussion \\
\hline \multicolumn{4}{|c|}{ Inférieur } \\
\hline Saïga & 22,4 & 9,0 & 8,9 \\
\hline Renne & 19,1 & 15,4 & 7,4 \\
\hline Cheval & 33,3 & 20,4 & 11,6 \\
\hline Bovinés & 19,7 & 13,1 & 7,8 \\
\hline \multicolumn{4}{|c|}{ Supérieur } \\
\hline Saïga & 41,1 & 11,3 & 6,8 \\
\hline Renne & 30,9 & 16,4 & 8,3 \\
\hline Cheval & 43,9 & 16,9 & 6,4 \\
\hline Bovinés & 36,4 & 14,5 & 12,1 \\
\hline
\end{tabular}

Tabl. 10 - SG (Trécolle) : répartition archéostratigraphique du pourcentage relatif des traces de boucherie observées sur les ossements d'antilope saïga, de renne, de cheval et de Bovinés (les stries longitudinales comprennent les stries allongées et des traces de raclage).

Table 10 - SG (Trécolle): distribution, by unit, of the relative percentage of butchery marks observed on bones of saiga antelope, reindeer, horse and large bovids (longitudinal marks include both long cutmarks as well as scraping marks). sur des carcasses de cerf (Costamagno, 2012). Se développant sur différentes faces, ces incisions témoignent d'un prélèvement disjoint de la peau des pattes et du tronc ${ }^{(15)}$.

Le décharnement est l'opération qui a laissé le plus de traces sur les ossements: tous les os charnus en portent. La désarticulation a aussi été très intense puisque toutes les articulations des membres, à l'exception de l'épaule, portent le témoignage de cette opération. De nombreuses traces relatives à l'extraction des tendons ont été identifiées sur les faces postérieures mais aussi antérieures des métacarpiens et métatarsiens. Les traces transversales identifiées sur les faces postérieures des phalanges 1 et 2 indiquent que les petits tendons étaient fréquemment recherchés, tout comme les sabots. Les os longs et les mandibules ont été systématiquement fracturés pour leur moelle. Cette source lipidique était aussi extraite des phalanges et en particulier des premières. Dans l'ensemble supérieur, $12 \%$ des phalanges 1 et $87,5 \%$ des phalanges 2 sont entières, tandis que dans l'ensemble inférieur, ce sont $23,2 \%$ des phalanges 1 et $81,8 \%$ des phalanges 2 .

Fig. 27 - SG (Trécolle) : schéma de boucherie de l'antilope saïga dans les ensembles inférieur et supérieur (DAO SC).

Fig. $27-S G$ (Trécolle): butchery pattern of saiga antelope in the lower and upper stratigraphic units (CAD SC).

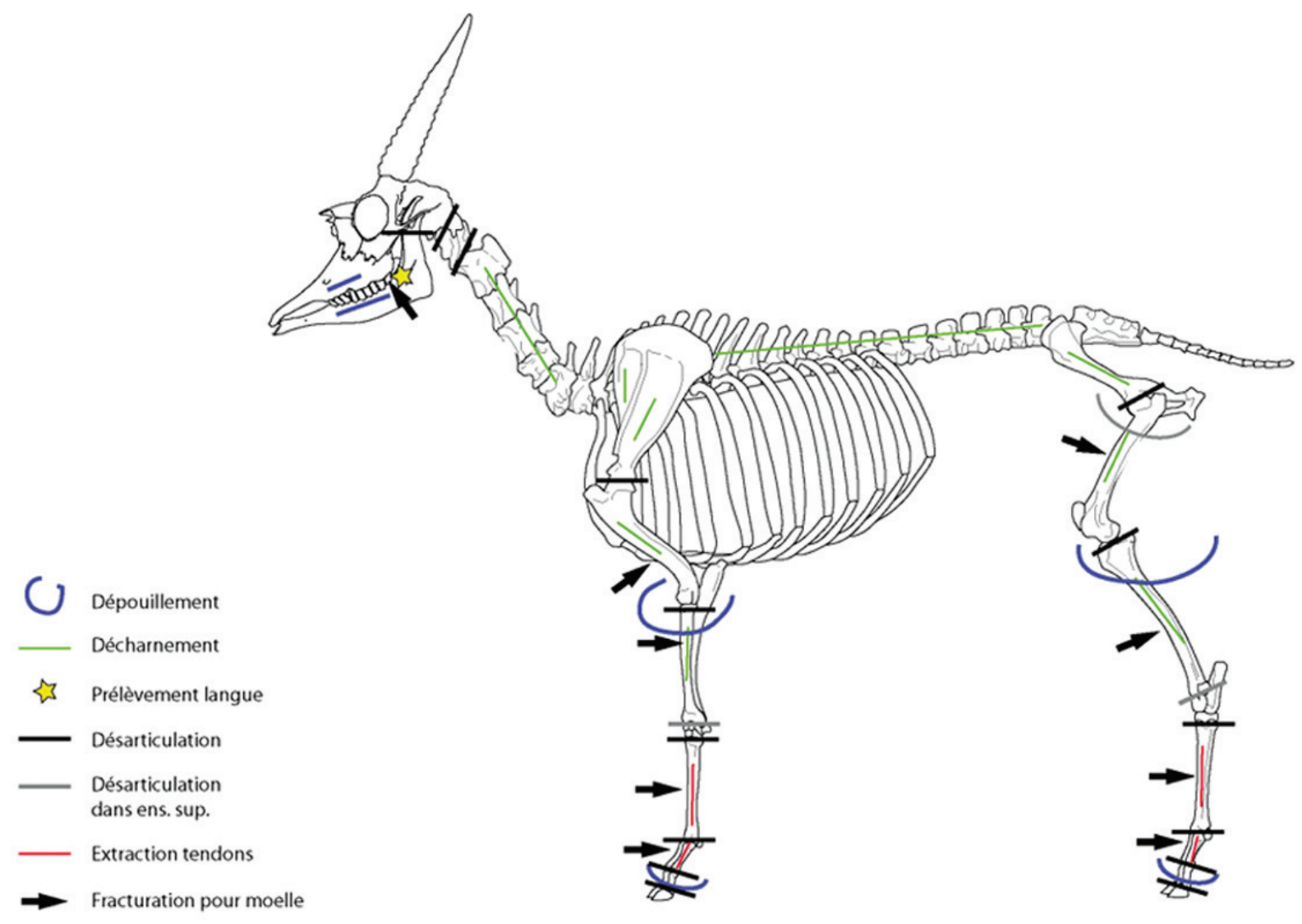

Référence squelette post-crânien : dessin M. Coutureau (INRAP), en collaboration avec V. Forest (@ 1996 ArcheoZoo.org, d'après R. Barone, 1976, Anatomie comparée des mammifères domestiques, 1. Ostéologie - atlas, Paris, Vigot, pl. 8, p. 23). Crâne : dessin J.-B. Mallye. 
Les phalanges 1, plus souvent fragmentées dans l'ensemble supérieur, attestent d'une recherche plus intensive de la moelle au Magdalénien moyen. L'étude entreprise sur l'emplacement des traces de percussion semble témoigner de techniques de percussion distinctes dans les deux ensembles (Costamagno, 1999, p. 186). Par exemple, dans l'ensemble inférieur, sur les radius, les coups ont souvent été assénés sur les faces médiale ou latérale alors que, dans l'ensemble supérieur, les points d'impact sont très fréquemment observés en face antérieure en dessous de l'extrémité proximale. Dans l'ensemble supérieur, la disposition des points d'impact témoigne d'un choix délibéré spécifique à chaque élément squelettique. Ces zones retenues sans lien strict avec la structure de l'os laissent à penser qu'il s'agit de schémas culturels nécessitant une transmission des savoir-faire (Masset, 2014).

\section{Les autres ongulés}

Dans les deux ensembles, la boucherie du renne est très similaire à celle de l'antilope saïga. Les incisions longitudinales observées sur les faces médiale et latérale des métapodes témoignent d'une récupération disjointe de la peau du tronc et de celle des pattes. Le décharnement des os longs est systématique et est réalisé avec des gestes transversaux ou longitudinaux. Les tendons sont aussi fréquemment prélevés. L'exploitation de la moelle est encore plus poussée que pour l'antilope saïga puisque, dans l'ensemble supérieur, même les phalanges 2 sont systématiquement fracturées. La chaîne opératoire est plus fragmentaire pour le cheval et les bovinés que pour l'antilope saïga ou le renne du fait du faible nombre de restes identifiés mais, globalement, les pratiques semblent similaires.

Outre les bois de rennes, nous avons vu précédemment que les ossements des quatre ongulés principaux ont été utilisés pour la confection d'outils. En dehors de l'exploitation des ongulés à des fins alimentaires ou techniques, les ossements ont aussi parfois été utilisés comme combustible (Costamagno, 1999; Costamagno et al., 2009). Plusieurs indices indiquent que cette pratique était plus fréquente dans l'ensemble inférieur (tabl. 11). Néanmoins, la bonne représentation des extrémités articulaires d'os longs et le faible pourcentage d'extrémités articulaires d'os longs brûlés d'antilope saïga montrent que, même dans cet ensemble, l'os n'était pas utilisé intensivement comme combustible.

\section{Exploitation des mésomammifères}

Le faible nombre de restes limite considérablement la reconstitution des chaînes opératoires de traitement des carcasses. Néanmoins de menus indices permettent d'apporter des informations sur les gestes et les produits recherchés.

Dans l'ensemble inférieur, quatre restes de renard portent des traces de l'activité humaine. Une incision sur une diaphyse de radius atteste du prélèvement des masses musculaires. Un fragment de diaphyse de fémur porte des stries longitudinales de raclage et montre une fracture dont la morphologie atteste de sa fracturation pour en extraire la moelle. D'autre part, des traces de brûlure visibles au

\begin{tabular}{|l|c|c|}
\cline { 2 - 3 } \multicolumn{1}{c|}{} & Inférieur & Supérieur \\
\hline$\%$ cheval brûlé & 6,2 & 3,6 \\
\hline$\%$ saïga brûlé & 7,9 & 2,5 \\
\hline$\%$ ext. arti. os longs saïga & 10,6 & 3,2 \\
\hline$\%$ os brûlé $>2 \mathrm{~cm}$ & 17,2 & 9,8 \\
\hline$\%$ os brûlé $<2 \mathrm{~cm}$ & 63,8 & 46 \\
\hline
\end{tabular}

Tabl. 11 - SG (Trécolle) : répartition archéostratigraphique du pourcentage d'os brûlés selon la taille des fragments et selon l'espèce.

Table 11 - SG (Trécolle): distribution, by unit, of the percentage of burnt bones according to fragment size and according to species.

niveau de l'apex d'une canine supérieure (fig. 28b) indiqueraient la cuisson de la tête à proximité d'une source de chaleur. Enfin, la racine d'une canine supérieure (fig. 28a) porte des traces de raclage possiblement réalisées lors des phases de préparation d'un élément de parure (Vanhaeren et D'Errico, 2003, fig. 29, nos 15 et 16). Le seul reste de lièvre portant des traces anthropogènes est un fragment de fémur (fig. $28 \mathrm{~g}$ ) sur lequel une strie de boucherie est visible près du grand trochanter.

Dans l'ensemble supérieur, une extrémité proximale d'ulna (fig. 28c) ainsi qu'une canine supérieure droite de renard roux (fig. 28d) sont incisées. Sur la première, la strie pourrait indiquer le prélèvement des masses musculaires ou correspondre à la désarticulation du coude. Sur la seconde, les traces, profondes et transverses, sont localisées au niveau du collet sur le bord lingual et présentent la particularité d'être associées à un fragment de silex fiché. Quant au lièvre, il semble avoir été exploité pour sa chair et sa moelle comme l'attestent des traces de découpe et la morphologie des bords de fracture observée sur une diaphyse tibiale.

Enfin, signalons, parmi les ossements non réattribués à l'un des deux ensembles retenus, un calcanéum de lièvre qui est marqué d'une strie liée au dépouillement (fig. 28f).

\section{Exploitation de l'avifaune}

Dans l'ensemble inférieur, la chouette harfang est documentée par de nombreux vestiges, essentiellement des os des membres et de la ceinture scapulaire (tabl. 12). Les manques et sous-représentations en certains éléments osseux peuvent s'expliquer par leur fragile constitution ou leur petite taille. Par ailleurs, la forte fragmentation des os longs entraîne une sous-représentation relative de certaines portions. En définitive, il est probable que les rapaces aient plutôt été introduits entiers sur le site.

Les stries observées sur plus d'une centaine de pièces et de rares traces de peeling témoignent qu'au moins une partie des carcasses a été réduite en quartiers et décharnée. La segmentation, qui a été réalisée à l'aide d'un tranchant ou par contrainte mécanique, concerne presque toutes les articulations de l'aile et de la patte (fig. 29). Toutes les parties charnues représentées par au moins un 

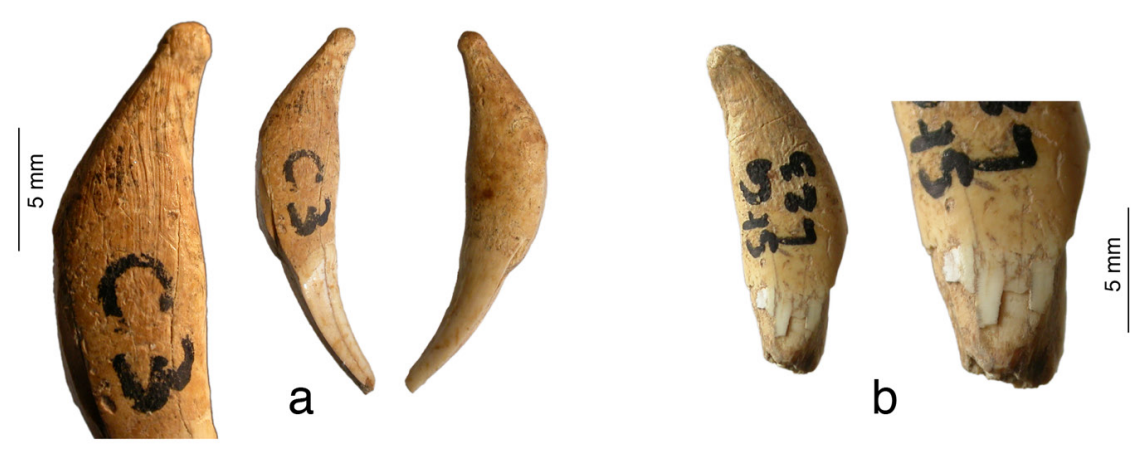

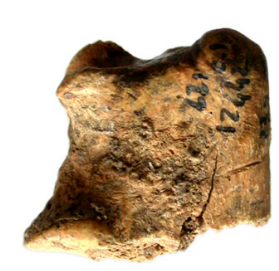

C

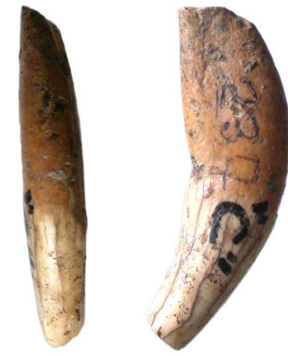

d
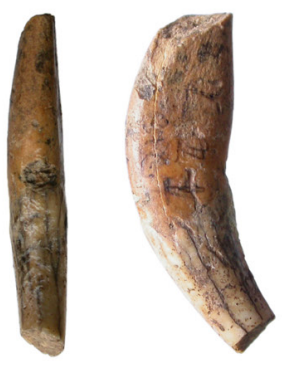
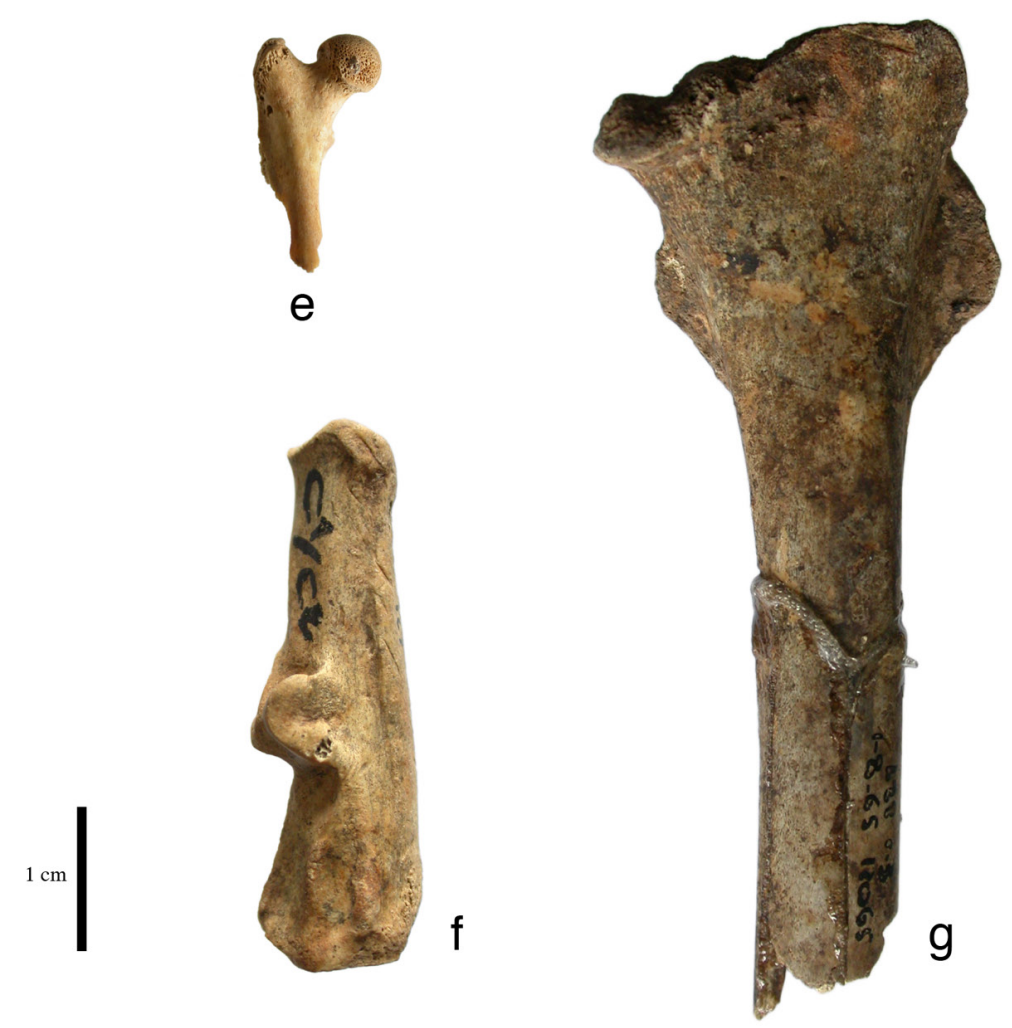

Fig. 28 - SG (Trécolle) : restes de mésomammifères portant des traces de prédation. a-d : renard; e : spermophile; f-g : lièvre. a : canine sup. droite avec trace de raclage sur sa racine; b : canine sup. droite avec trace de chauffe (apex de la couronne); $\mathrm{c}$ fragment proximal d'ulna gauche avec stries de découpe; $d$ : canine sup. droite avec traces de raclage au niveau de la racine et le long de la couronne, un fragment de silex est fiché sur la face distale de la canine au niveau du collet; e : fémur droit portant des traces de digestion; f : calcanéum gauche avec trace de découpe oblique le long du corps de l'os; $g$ : fémur gauche de lièvre avec trace de découpe au dessous du grand trochanter (DAO JBM).

Fig. 28 -SG (Trécolle): small and medium mammals remains with predation marks. a-d: fox; e: ground squirrel; f-g: artic hare. a: upper right canine with scrapping marks on its root; $b$ : upper right canine with burn marks at the apex; $c$ : left ulna - proximal part - with cut marks; $d$ : upper right canine with scrapping marks on its root and on the its crown, a flint fragment is embedded in the distal part of the crown near its neck e: right femur with digestion marks; f: left calcaneus, some cut marks are visible along the lateral face; g: left femur of a hare, a cut mark is clearly visible at the base of the great trochanter (CAD JBM). 
NRD $\mid$ NME $\mid$ NRD strie $\quad \%$ survie

\begin{tabular}{|l|c|c|c|c|}
\hline Carré & 3 & 3 & 0 & 6,8 \\
\hline Prémaxillaire & 2 & 2 & 0 & 4,5 \\
\hline Vertèbres & 8 & 8 & 0 & 1,4 \\
\hline Coracoïde & 28 & 16 & 3 & 36,4 \\
\hline Scapula & 21 & 17 & 4 & 38,6 \\
\hline Humérus & 30 & 15 & 7 & 34,1 \\
\hline Ulna & 24 & 19 & 13 & 43,2 \\
\hline Radius & 120 & 25 & 17 & 56,8 \\
\hline Ulnaire & 1 & 1 & 0 & 2,3 \\
\hline Carpométacarpe & 24 & 15 & 2 & 34,1 \\
\hline Phalanges aile & 5 & 5 & 0 & 2,8 \\
\hline Fémur & 25 & 15 & 5 & 34,1 \\
\hline Tibiotarse & 54 & 32 & 8 & 72,7 \\
\hline Fibula & 13 & 13 & 2 & 29,5 \\
\hline Tarsométatarse & 15 & 10 & 1 & 22,7 \\
\hline pha p I1 & 22 & 22 & 9 & 50,0 \\
\hline pha p I2 & 44 & 44 & 6 & 100,0 \\
\hline pha p II1 & 6 & 6 & 2 & 13,6 \\
\hline pha p II2 & 19 & 19 & 3 & 43,2 \\
\hline pha p II3 & 22 & 22 & 2 & 50,0 \\
\hline pha p III1 & 4 & 4 & 1 & 9,1 \\
\hline pha p III2 & 12 & 12 & 2 & 27,3 \\
\hline pha p III3 & 16 & 16 & 3 & 36,4 \\
\hline pha p III4 & 31 & 31 & 2 & 70,5 \\
\hline pha p IV1 & 2 & 2 & 0 & 4,5 \\
\hline pha p IV2 & 2 & 2 & 0 & 4,5 \\
\hline pha p IV4 & 24 & 24 & 5 & 54,5 \\
\hline pha p IV5 & 23 & 23 & 2 & 52,3 \\
\hline pha p pen & 14 & - & 4 & - \\
\hline pha p grif & - & 0 & - \\
\hline Total & & 103 & \\
\hline
\end{tabular}

Tabl. 12 - SG (Trécolle, ensemble inférieur) : décomptes des NRD, NME, NRD portant des stries et pourcentage de survie des éléments anatomiques de Chouette harfang (pha p I1 à IV5 $=$ phalanges des pattes suivie du numéro du doigt et du rang).

Table 12 - SG (Trécolle, lower stratigraphic unit): values of NISP, MNE, NISP of cutmarked bones and percentage presence (or percentage likelihood of survival?), of each anatomical element for snowy owl (pha p II à IV5 = leg phalanxes followed by the digit number and rank).

élément anatomique portent des traces de prélèvement de la viande. Des stries présentes sur le carpométacarpe, le tarsométatarse et des pénultièmes phalanges du pied indiquent possiblement la recherche des plumes, grandes et petites. Bien que d'interprétation ambiguë, les stries longitudinales de raclage qui sont visibles sur des fragments d'ulnas et de radius pourraient être liées au travail de l'os. La présence d'un fragment de radius décoré et de fragments d'industrie osseuse sur os d'oiseau de taille de la chouette des neiges tend à soutenir cette hypothèse. Le traitement des carcasses de harfang semble orienté vers différents produits : viande, os, plumes, phalanges du pied et notamment les griffes. Parmi les autres oiseaux utilisés, citons les lagopèdes, et en particulier un radius qui porte des stries de raclage dont la finalité nous échappe. Enfin, l'oie livre un seul ossement, une extrémité distale d'humérus ayant servi à la confection de baguettes (voir supra). Dans l'ensemble attribué au Magdalénien inférieur, les ressources aviaires participent donc à plusieurs sphères d'activités (alimentaire, technique, symbolique) de la vie des chasseurs-cueilleurs.

Dans l'ensemble supérieur, le grand corbeau fait une apparition discrète dans le tableau de chasse. Le prélèvement de sa viande est visible sur un humérus et un fémur qui montre par ailleurs la preuve d'une découpe au niveau du genou. Les restes de harfang portant des traces sont moins nombreux que dans l'ensemble inférieur (NISP strie $=25$ ) et le schéma de traitement qui en découle nous apparaît plus partiel. Néanmoins, la désarticulation des carcasses en plusieurs points de la patte et des ailes ainsi que le prélèvement de la viande sont documentés. L'intérêt pour les phalanges du pied et les plumes ainsi que l'utilisation des os (ulna) comme matière première semble se maintenir.

\section{Comparaisons}

Dans les deux ensembles des fouilles Trécolle, le spectre faunique est dominé par l'antilope saïga comme dans plusieurs sites régionaux de cette période (Delpech, 1989; Costamagno, 2001). La chouette harfang tient le deuxième rôle avant le renne dans l'ensemble inférieur et diminue au profit du renne et du cheval au Magdalénien moyen. Le spectre est complété notamment par des Bovinés. Ce taxon peu fréquent à $\mathrm{SG}$ a été parfois une proie abondamment chassée par les Magdaléniens de Gironde que ce soit dans la phase ancienne ou la phase moyenne. Par exemple au Roc-de-Marcamps (locus 1, couches 4-6), le spectre faunique est dominée par le bison (Slott-Möller, 1988) tout comme à Fongaban où c'est quasiment la seule espèce présente (Delpech, 1972; Martin, 2013). Quelques restes de renard et lièvre indiquent également une chasse de ces petits gibiers. Si l'on note une similitude de saisonnalité entre les deux ensembles, on remarque néanmoins une différence dans les modes et l'intensité de la récupération de la moelle, renvoyant à des fonctions de site différentes. Peu de données archéozoologiques sont disponibles mais nous pouvons mentionner quelques comparaisons permettant de souligner la variabilité des spectres selon les biotopes.

$\mathrm{Au}$ Taillis des Coteaux, les groupes du Magdalénien inférieur de AG-IIIa comme du Magdalénien moyen (ensemble II) ont chassé essentiellement du renne puis du cheval et du Boviné (Griggo in Primault et al., 2010). La question de la consommation du poisson au Magdalénien inférieur est encore débattue (Primault et al., 2010; Rambaud et al., 2011). À l'abri Gandil, l'étude paléontologique de la faune du Magdalénien inférieur livre un spectre faunique dominé par le renne, suivi du couple cha- 


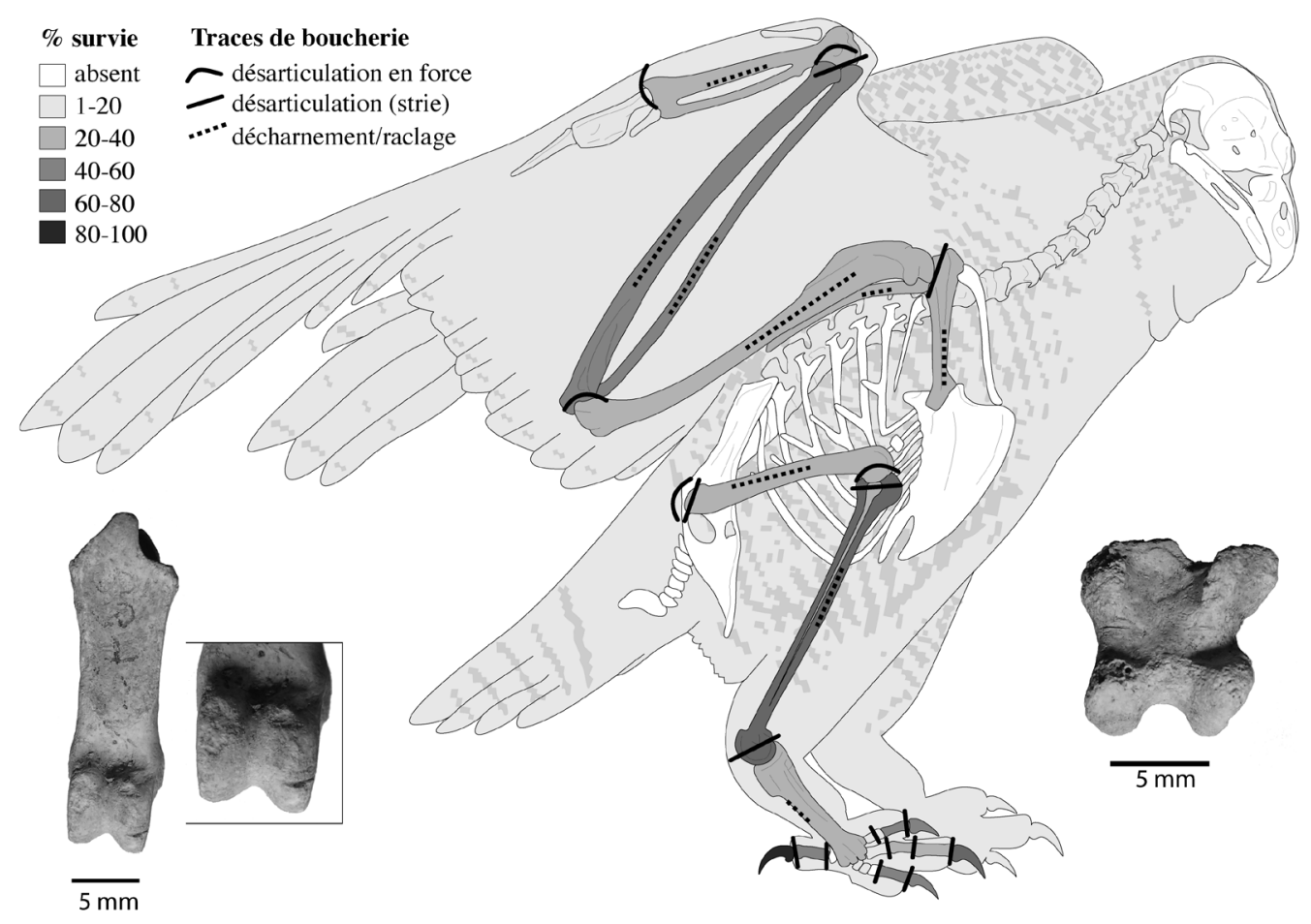

Référence : Dessin Michel Coutureau (Inrap), avec la collaboration de Véronique Laroulandie (CNRS) - 10/2007

Fig. 29 - SG (ensemble inférieur) : schéma synthétique de la conservation squelettique et du traitement des carcasses de chouette harfang (DAO VL).

Fig. 29 - SG SG (lower stratigraphic unit): schematic drawing summarizing skeletal preservation and carcass processing of snowy owl (CAD VL).

mois-bouquetin et de quelques restes de cheval (Griggo, 1997). Nous pouvons rappeler la présence de trois restes de chouette harfang dont l'origine anthropogénique reste à préciser. L'ensemble supérieur des fouilles d'E. Ladier, attribuable au Magdalénien moyen sur la présence de lamelles scalènes, est également dominé par le renne, suivi tantôt du couple chamois-bouquetin tantôt du cheval en c. 5 - couche qui a également livré le seul reste de saïga des fouilles récentes (Griggo, 1997). Au Petit Cloup Barrat, en l'état des travaux sur la couche 4, le spectre est dominé par le renne (Castel in Ducasse et al., 2011). Aux Scilles, c'est également le renne mais cette fois suivi des Bovinés, puis du chamois, cheval, cerf et deux restes de saïga (Letourneux et David in Langlais et al., 2010). Dans les niveaux attribués au Magdalénien moyen de Troubat (c. 13), c'est aussi le renne qui domine (Costamagno, inédit). Rappelons par ailleurs que le bouquetin, absent du régime alimentaire des groupes de SG, est représenté sur une gravure dans la couche B du Grand Abri fouillé par R. Blanchard (Blanchard et al., 1972, fig. 15). Cette image n'est pas sans rappeler une gravure de bouquetin provenant de la c. 2 de l'abri Gandil (Ladier, 2002, fig. 7).

\section{Nouvelles dates radiométriques}

$\mathrm{D}$ ix nouvelles dates ${ }^{14} \mathrm{C}$ AMS ont été obtenues sur le matériel des fouilles Trécolle au sein de trois laboratoires différents (fig. 30, tabl. 13). Pour le complexe inférieur, dans le cadre du programme ARTEMIS (labo- ratoires de Lyon et Saclay), trois résultats ont été obtenus sur espèces : chouette harfang, antilope saïga et Bovinés et trois autres sur industrie osseuse : un déchet de façonnage, un fragment de pointe et un fragment d'outil mousse. Pour l'ensemble supérieur, une date sur renne a été obtenue au laboratoire de Poznan dans le cadre d'un projet Fyssen. Dans le cadre du projet Magdatis, trois échantillons envoyés au laboratoire ORAU (Oxford Radiocarbon Accelerator Unit, University of Oxford) ont fonctionné : un os de Boviné, un os de chouette harfang et un déchet de débitage sur perche $\mathrm{C}$ (voir BarshaySzmidt et al., en préparation, pour une description de la méthodologie utilisée). Néanmoins, l'ensemble supérieur de ce site a été difficile à dater puisque cinq ossements et six éléments d'industrie osseuse en bois de cervidé de la fouille Trécolle ainsi que deux éléments en bois de renne de la fouille Blanchard soumis pour datation ont échoué. Dans tous les cas sauf un, ce fut en raison de la faible quantité de collagène (ou d'azote qui donne une bonne indication de la présence de collagène, Brock et al., 2010). Dans un cas le rapport $\mathrm{C} / \mathrm{N}$ fut trop élevé. Mise à part la date sur harfang, dont le code OxA-X indique une fiabilité moindre (taux de collagène sous la limite optimale), on observe une discontinuité chronologique entre les deux ensembles de la séquence Trécolle (fig. 30, tabl. 13). Ce possible hiatus d'occupation dans le talus, d'environ 700 ans, est en partie comblé par deux dates obtenues pour la terrasse supérieure de SG (fouilles Blanchard) : la sépulture et un os provenant du « magma ». 


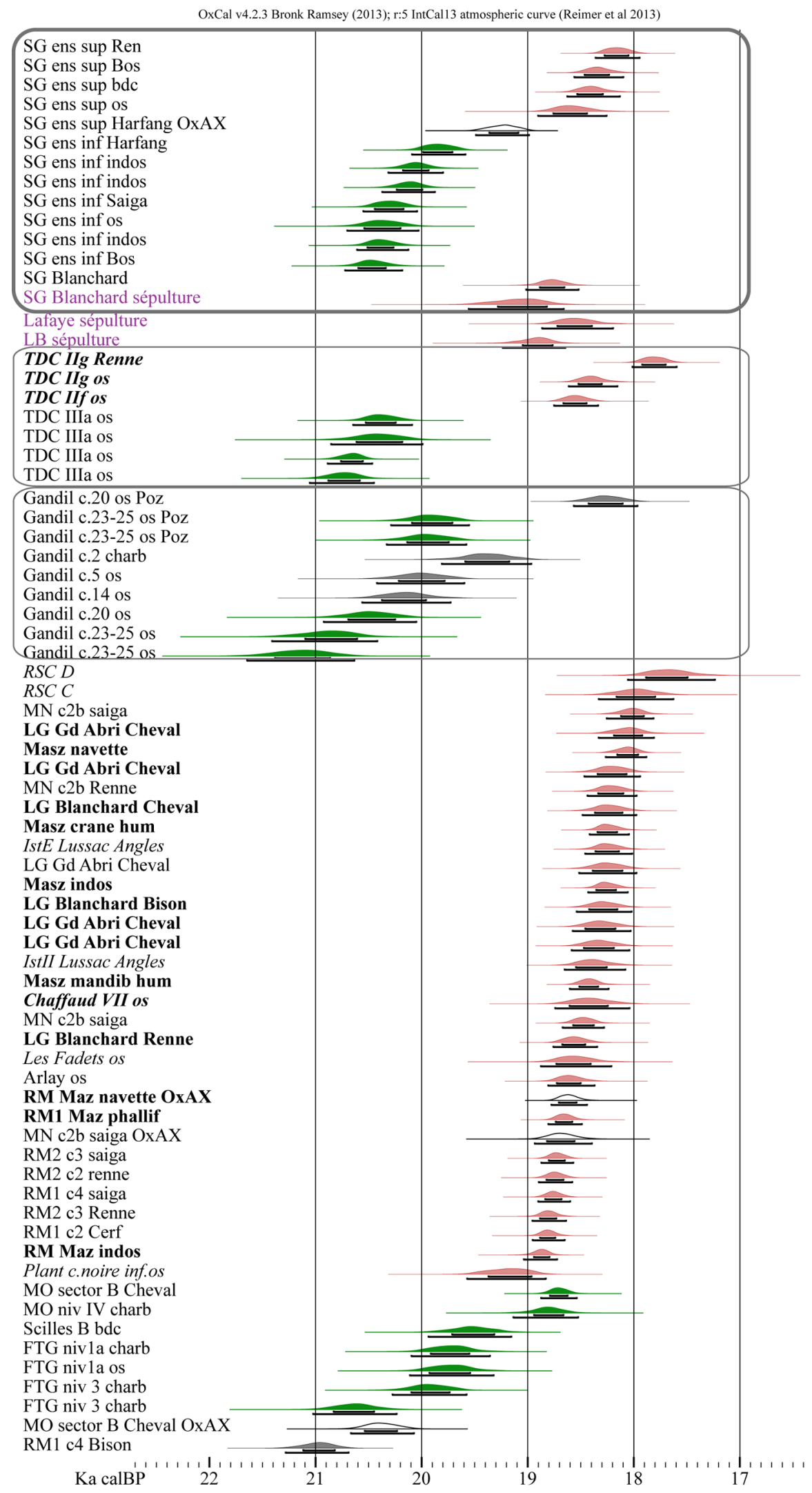

Fig. 30 - Datations ${ }^{14} \mathrm{C}$ AMS des séquences de SG, du Taillis des Coteaux et de Gandil. En violet : dates directes sur os humain en contexte sépulcral; en rouge : Magdalénien moyen ancien (gras : à navettes; italique : à pointes de Lussac-Angles); en vert : Magdalénien inférieur; en grisé : indéterminé et dates problématiques (pour les légendes des sites voir le tableau 13).

Fig. $30-{ }^{14} \mathrm{C}$ AMS dates obtained on materials from SG, Taillis des Coteaux and Gandil. In purple: dates taken directly from a human bone within a burial context; in red: early Middle Magdalenian (in bold: navette facies; in italics: Lussac-Angles facies); in green: Lower Magdalenian; in grey: indeterminate and problematic dates (see table 13 for site listing). 


\begin{tabular}{|c|c|c|c|c|c|c|}
\hline Site & Couche & Nature & Date & Écart & Laboratoire & Bibliographie \\
\hline Saint-Germain & ens. sup. & renne & 14940 & 70 & Poz-52970 & Barshay-Szmidt et al., en prep. \\
\hline Saint-Germain & ens. sup. & Bos-bison & 15090 & 75 & OxA-26655 & Barshay-Szmidt et al., en prep. \\
\hline Saint-Germain & ens. sup. & bdc & 15140 & 90 & OxA26486 & Barshay-Szmidt et al., en prep. \\
\hline Saint-Germain & ens. sup. & os & 15330 & 150 & OxA7345-Ly615 & Lenoir, 2000 \\
\hline Saint-Germain & ens. sup. & harfang & 15940 & 80 & OxA-X-2503-18 & Barshay-Szmidt et al., en prep. \\
\hline Saint-Germain & ens. inf. & harfang & 16450 & 90 & Lyon-10175 (SacA 32842) & Barshay-Szmidt et al., en prep. \\
\hline Saint-Germain & ens. inf. & indos & 16620 & 80 & Ly10230/SacA33705 & Barshay-Szmidt et al., en prep. \\
\hline Saint-Germain & ens. inf. & indos & 16670 & 80 & Ly10231/SacA33706 & Barshay-Szmidt et al., en prep. \\
\hline Saint-Germain & ens. inf. & saiga & 16830 & 90 & Lyon-10174 (SacA 32841) & Barshay-Szmidt et al., en prep. \\
\hline Saint-Germain & ens. inf. & os & 16890 & 130 & OxA7260/Ly617 & Lenoir et al., 1994 \\
\hline Saint-Germain & ens. inf. & indos & 16900 & 80 & Ly10232/SacA33707 & Barshay-Szmidt et al., en prep. \\
\hline Saint-Germain & ens. inf. & Bos-bison & 16970 & 90 & Lyon-10176 (SacA 32843) & Barshay-Szmidt et al., en prep. \\
\hline Saint-Germain & $\mathrm{SG} «$ magma » & os & 15510 & 120 & OxA7258/Ly614 & Lenoir, 2000 \\
\hline Saint-Germain & Blanchard sép. & os humain & 15780 & 200 & GifA95456 & Gambier et al., 2000 \\
\hline Lafaye & sépulture & os humain & 15290 & 150 & GifA95047 & Gambier et al., 2000 \\
\hline Laugerie Basse & sépulture & os humain & 15660 & 130 & GifA94204 & Gambier et al., 2000 \\
\hline Taillis des coteaux & IIg & os renne & 14630 & 75 & Ly3876 & Primault et al., 2007 \\
\hline Taillis des coteaux & IIg & os & 15140 & 80 & Ly6410 & Airvaux et al., 2012 \\
\hline Taillis des coteaux & IIf & os & 15280 & 90 & Ly6408 & Airvaux et al., 2012 \\
\hline Taillis des coteaux & IIIa & os & 16900 & 100 & Ly6409 & Primault, dir., 2010 \\
\hline Taillis des coteaux & IIIa & os & 16920 & 170 & Ly2264 & Primault et al., 2007 \\
\hline Taillis des coteaux & IIIa & os & 17130 & 65 & OxA12180 & Primault et al., 2007 \\
\hline Taillis des coteaux & IIIa & os & 17190 & 110 & Ly6406 & Primault et al., 2007 \\
\hline Gandil & c. $20 \mathrm{Poz}$ & os & 15033 & 120 & Ly2483 (Poz) & Langlais et al., 2007 \\
\hline Gandil & c. $23-25 \mathrm{Poz}$ & os & 16507 & 144 & Ly2485(Poz) & Langlais et al., 2007 \\
\hline Gandil & c. $23-25 \mathrm{Poz}$ & os & 16538 & 144 & Ly2484 (Poz) & Langlais et al., 2007 \\
\hline Gandil & c. 2 & charbon & 16070 & 160 & GifA 93238 & Tisnerat Laborde et al., 1997 \\
\hline Gandil & c. 5 & os & 16580 & 160 & GifA 96350 & Tisnerat Laborde et al., 1997 \\
\hline Gandil & c. 14 & os & 16700 & 160 & GifA 96351 & Tisnerat Laborde et al., 1997 \\
\hline Gandil & c. 20 & os & 16980 & 170 & GifA 96416 & Tisnerat Laborde et al., 1997 \\
\hline Gandil & c. $23-25$ & os & 17290 & 180 & GifA 97307 & Tisnerat Laborde et al., 1997 \\
\hline Gandil & c. $23-25$ & os & 17480 & 180 & GifA 96417 & Tisnerat Laborde et al., 1997 \\
\hline Roc aux Sorciers & $\mathrm{D}$ & os & 14510 & 160 & GifA94191 & Airvaux et al., 2012 \\
\hline Roc aux Sorciers & $\mathrm{C}$ & os & 14770 & 140 & GifA94190 & Airvaux et al., 2012 \\
\hline Moulin Neuf & c. $2 b$ & Saiga & 14810 & 75 & OxA26656 & Barshay-Szmidt et al., en prep. \\
\hline La Garenne & Grand Abri & cheval & 14840 & 100 & ETH29157 & Despriée et al., 2009 \\
\hline Maszicka & - & navette & 14855 & 60 & KIA39225 & Kozsłowski et al., 2012 \\
\hline La Garenne & Grandd Abri & cheval & 14980 & 100 & ETH29154 & Despriée et al., 2009 \\
\hline Moulin Neuf & c. $2 b$ & renne & 14990 & 80 & Poz52971 & Barshay-Szmidt et al., en prep. \\
\hline La Garenne & Blanchard & cheval & 15010 & 90 & ETH28494 & Despriée et al., 2009 \\
\hline Maszicka & - & os humain & 15015 & 50 & KIA39227 & Kozsłowski et al., 2012 \\
\hline Isturitz & E & Lussac-Angles & 15020 & 70 & OxA28083 & Barshay-Szmidt et al., en prep. \\
\hline La Garenne & Grand Abri & cheval & 15020 & 100 & ETH29158 & Despriée et al., 2009 \\
\hline Maszicka & - & pointe indos & 15025 & 50 & KIA39226 & Kozsłowski et al., 2012 \\
\hline La Garenne & Blanchard & bison & 15050 & 90 & ETH28493 & Despriée et al., 2009 \\
\hline La Garenne & Grand Abri & cheval & 15070 & 100 & ETH29156 & Despriée et al., 2009 \\
\hline La Garenne & Grand Abri & cheval & 15080 & 100 & ETH29155 & Despriée et al., 2009 \\
\hline
\end{tabular}

Tabl. 13 - Liste des dates ${ }^{14} \mathrm{C}$ AMS de la figure 31. Le code OxA-X indique des datations considérées moins fiables par le laboratoire concerné (masse ou taux de collagène faible), mais pas rejetées par celui-ci.

Table 13 - List of ${ }^{14} \mathrm{C}$ AMS dates depicted in figure 31. OxA-X codes indicate dates considered slightly less reliable by that laboratory (collagen mass or percentage are low), but not rejected by that laboratory. 


\begin{tabular}{|c|c|c|c|c|c|c|}
\hline Site & Couche & Nature & Date & Écart & Laboratoire & Bibliographie \\
\hline Isturitz & II & Lussac-Angles & 15130 & 110 & OxA19836 & Szmidt et al., 2009 \\
\hline Maszicka & - & os humain & 15155 & 60 & KIA39228 & Kozsłowski et al., 2012 \\
\hline Chaffaud & VII & os & 15160 & 150 & Gif7357 & Airvaux et al., 2012 \\
\hline Moulin Neuf & c. $2 b$ & saiga & 15205 & 75 & OxA26659 & Barshay-Szmidt et al., en prep. \\
\hline La Garenne & Blanchard & renne & 15290 & 90 & ETH28492 & Despriée et al., 2009 \\
\hline Les Fadets & - & os & 15300 & 150 & Gif7353 & Airvaux et al., 2012 \\
\hline Arlay & - & os & 15335 & 100 & Ly3161Poz & Cupillard et Welté, 2006 \\
\hline Marcamps 1 & Maziaud & navette & 15340 & 70 & OxA-X-248219 & Barshay-Szmidt et al., en prep. \\
\hline Marcamps 1 & Maziaud & phalliforme & 15380 & 70 & OxA27394 & Barshay-Szmidt et al., en prep. \\
\hline Moulin Neuf & c. $2 b$ & saiga & 15420 & 130 & OxA-X-248219 & Barshay-Szmidt et al., en prep. \\
\hline Marcamps 2 & c. 3 & saiga & 15460 & 70 & OxA28089 & Barshay-Szmidt et al., en prep. \\
\hline Marcamps 2 & c. 2 & renne & 15480 & 75 & OxA28092 & Barshay-Szmidt et al., en prep. \\
\hline Marcamps 1 & c. 4 & saiga & 15495 & 70 & OxA26664 & Barshay-Szmidt et al., en prep. \\
\hline Marcamps 2 & c. 3 & renne & 15550 & 75 & OxA28090 & Barshay-Szmidt et al., en prep. \\
\hline Marcamps 1 & c. 2 & cerf & 15555 & 70 & OxA26662 & Barshay-Szmidt et al., en prep. \\
\hline Marcamps 1 & Maziaud & biseau double & 15630 & 70 & OxA26665 & Barshay-Szmidt et al., en prep. \\
\hline Plantade & c. noire inf. & os & 15890 & 160 & GifA94185 & Tisnerat Laborde et al., 1997 \\
\hline Montlleo & sector B & dent cheval & 15440 & 80 & OxA9017 & Fullola et al., 2012 \\
\hline Montlleo & niv. IV & charbon & 15550 & 140 & OxA14034 & Fullola et al., 2012 \\
\hline Scilles & $\mathrm{B}$ & bdc & 16180 & 140 & TO 13555 & Pétillon et al., 2008 \\
\hline Fontgrasse & niv. 1a & charbon & 16338 & 143 & Erl 8925 & Bazile, 2006 \\
\hline Fontgrasse & niv. 1a & os & 16338 & 153 & Erl 8928 & Bazile, 2006 \\
\hline Fontgrasse & niv. 3 & charbon & 16518 & 133 & Erl 8936 & Bazile, 2006 \\
\hline Fontgrasse & niv. 4 & charbon & 17100 & 144 & Erl 8937 & Bazile, 2006 \\
\hline Montlleo & sector B & dent cheval & 16900 & 110 & OxA-X-223452 & Fullola et al., 2012 \\
\hline Marcamps 1 & c. 4 & bison & 17380 & 90 & OxA26663 & Barshay-Szmidt et al., en prep. \\
\hline
\end{tabular}

Tabl. 13 (suite) - Liste des dates ${ }^{14} \mathrm{C}$ AMS de la figure 31.

Table 13 (end) - List of ${ }^{14} \mathrm{C}$ AMS dates depicted in figure 31.

\section{SYNTHESE ET DISCUSSION}

$\mathrm{E}$ n considérant la zone fouillée comme un échantillon représentatif de ce secteur, la séquence archéologique de SG (fouilles Trécolle) livre des éléments témoignant de changements et de continuités dans le comportement techno-économique des chasseurs magdaléniens. Ces faits offrent également la possibilité de discuter à une plus vaste échelle des processus de mise en place des normes du Magdalénien dans sa période classique (phases moyenne et supérieure), notamment au travers de la recomposition de l'armement de chasse et la standardisation des supports d'outils domestiques mais également le traitement des gibiers. Les occupations magdaléniennes des deux ensembles documentent l'exploitation durant la bonne saison d'un gibier dominé par le saïga suivi du renne et du harfang. Toutefois, des différences dans les stratégies de chasse sont à noter comme la composition des hardes d'antilopes chassées (groupes de femelles dans l'ensemble inférieur et hardes mixtes au Magdalénien moyen), mais aussi l'intensité (plus forte au Magdalénien moyen) et les modes de récupération de la moelle. La chasse aux oiseaux, en particulier de la chouette harfang, est une donnée nouvelle pour cette période. Elle témoigne à SG d'une continuité entre les deux ensembles, bien que sa place apparaisse plus réduite au Magdalénien moyen. Les mésomammifères demeurent marginaux dans toute la séquence. Sur la base d'analyses isotopiques menées sur des ossements humains issus de la sépulture de SG, le bison constituerait la principale source carnée de l'individu inhumé, relativisant, selon les auteurs, l'importance de l'antilope saïga dans le régime alimentaire des groupes du Magdalénien moyen ayant occupé le site (Drucker et al., 2005). Si le bison est effectivement une proie abondamment chassée sur certains sites girondins (Delpech, 1972; Slott-Möller, 1988), ses restes sont, en revanche, rares à SG. Certes, il est possible d'envisager, comme les auteurs, que la viande de bison ait été introduite désossée, expliquant cette distorsion quantitative entre bison et saïga. Plusieurs études ont montré l'importance que revêt la graisse pour les chasseurs-cueilleurs paléolithiques (voir par exemple Speth et Spielmann, 1983; Costamagno et Rigaud, 2014; Soulier et al., 2014). Dans ce cadre, l'exploitation intensive de la graisse à SG qui est attestée par la fracturation des phalanges de saïga et de renne ne nous semble pas compatible avec un rejet systématique des os longs de bison à moins d'admettre que ce gibier 
n'ait été chassé à une saison distincte de la saison d'occupation du site, renvoyant alors à une consommation différée de cette ressource sous la forme de viande séchée. Sur la base des données disponibles, il est néanmoins difficile d'aller aussi loin dans les interprétations. L'individu inhumé à $\mathrm{SG}$, dont la date directe montre un décalage chronologique avec la séquence Trécolle (fig. 30), était-il issu du même groupe qui avait occupé le contrebas du site au Magdalénien moyen? Peut-on mettre en relation la sépulture avec le dit « magma » d'ossements qui livre justement des restes de Bovinés? A-t-il été inhumé en dehors de la saison de chasse à l'antilope saïga? Les premiers résultats cémentochronologiques obtenues sur des dents de bison issus de sites magdaléniens girondins indiquent un faible recouvrement saisonnier du saïga et du bison (Pubert et Rendu, inédit) qui pourrait témoigner d'une complémentarité entre ces deux taxons. Toutefois, de nouvelles analyses sont nécessaires. Quelles que soient les raisons pouvant expliquer les différences entre analyses isotopiques et spectres fauniques, l'antilope saïga, d'un point de vue tant alimentaire que technique, a joué un rôle crucial pour ces groupes tout au long de la séquence Trécolle.

L'industrie osseuse ne montre pas de rupture dans le choix des matières premières et des techniques de débitage des baguettes par double rainurage longitudinal. En revanche, on note un changement dans le calibre des supports (plus important au Magdalénien moyen). L'évolution des types de base des pointes osseuses (base pleine du Magdalénien inférieur vs base biseautée ou en languette du Magdalénien moyen) signe une évolution des modes d'emmanchement. Cette recomposition des armements osseux peut être corrélée avec la transformation des armatures lithiques, passant des microlamelles à dos de profil convexe du Magdalénien inférieur aux grandes lamelles rectilignes tronquées ou denticulées du Magdalénien moyen. Entre les deux ensembles, on remarque une systématisation de la lame comme support d'outil domestique dès les premiers temps du Magdalénien moyen. Cette évolution technique ne s'est pas traduite dans l'acquisition des silex, malgré un apport plus important dans l'ensemble supérieur de silex des Charentes sous la forme de supports. Cette relative monotonie lithologique fortement ancrée sur les ressources locales, dans le Magdalénien tant inférieur que moyen des fouilles Trécolle, tranche non seulement avec le Magdalénien moyen des fouilles Blanchard ou Mirande, qui livre notamment de nombreux silex de la vallée du Cher (Langlais, observation personnelle), mais aussi avec les sites du Magdalénien moyen ancien girondin (Sécher, 2013; Langlais et al., soumis).

En définitive, la séquence Trécolle témoigne d'une évolution au cours des premiers temps du Magdalénien marquée par un continuum économique et des changements techniques. On peut également proposer que les différences notées entre le Magdalénien moyen du talus et de la couche A de Blanchard avec celui de la couche $B$ et de la terrasse supérieure relèvent en partie de la fonction différente des occupations, notamment dans certaines activités économiques (production de grandes lames, importations de silex lointains, traitement des carcasses ?) et symboliques (gravures pariétales, sépultures).

À une échelle plus vaste, les séquences potentiellement comparables à celle de SG ont pour la plupart été fouillées dans la première moitié du $\mathrm{XX}^{\mathrm{e}}$ siècle avec des méthodes d'enregistrement peu précises. Néanmoins, la révision récente de séries attribuées anciennement au Magdalénien II - telles que Laugerie-Haute, Chancelade, La Chaire-à-Calvin, Le Cap-Blanc ou Reverdit - livre de nouvelles données sur le matériel et le contexte radiométrique pour discuter des premières phases du Magdalénien dans ces gisements (Delage, inédit; Langlais, inédit; Bourdier, 2010; Bourdier et al., 2014a et b). Le site de Gabillou offre de fortes similitudes avec SG mais la révision du matériel reste à faire. D'autres archéostratigraphies de comparaison peuvent être mobilisées : le Taillis des Coteaux (fouilles J. Primault), l'abri Gandil (fouilles E. Ladier) et le Petit Cloup Barrat (fouilles J.-C. Castel). La séquence supérieure du Taillis des Coteaux, avec l'ensemble IIIa attribué au Magdalénien inférieur et daté entre 21 et $20 \mathrm{Ka}$ cal. BP et l'ensemble II attribué au Magdalénien moyen et daté entre 18,7 et $17,8 \mathrm{Ka}$ cal. BP, offre une forte similitude avec SG-Trécolle, tant dans les équipements (voir ci-dessus) que dans le cadre radiométrique (fig. 30; Primault et al., 2010; Airvaux et al., 2012; Astier, 2014). Pour l'abri Gandil, les deux séries de dates obtenues dans deux laboratoires différents (Gif et Lyon-Poznan) sont difficilement comparables en l'état (Langlais, 2007a). En outre, la date de Poznan obtenue pour la couche 20, attribuée au Magdalénien inférieur, apparaît bien récente au regard non seulement des dates faites à Gif-sur-Yvette mais aussi des séries de comparaison (fig. 30). Toutefois, les équipements lithiques et osseux montrent des rapprochements significatifs avec la séquence Trécolle (voir ci-dessus). Enfin, le Petit Cloup Barrat livre un ensemble archéologique et des dates compatibles avec du Magdalénien inférieur mais sa caractérisation est en cours et nécessite encore une meilleure compréhension de ses rapports stratigraphiques tant avec le Badegoulien qu'avec le Magdalénien moyen. La confrontation des données radiométriques $^{(16)}$ (fig. 30) montre une certaine unité du Magdalénien inférieur autour de l'intervalle 21-18,8 Ka cal. BP. Quant au Magdalénien moyen ancien, malgré des chevauchements potentiellement liés à des arythmies géographiques (fig. 1, fig. 30), il peut être calé autour de 19-18 Ka cal. BP.

Le Magdalénien inférieur apparaît de prime abord comme une phase de stabilité des équipements entre environ 21 et $19 \mathrm{Ka}$ cal. BP (osseux : débitage par rainurage longitudinal, pointes à base pleine; ou lithiques : productions microlamellaires, variabilité morphométrique de l'outillage domestique en silex). Les travaux menés sur la diversité typo-technologique des armatures (micro)lamellaires (voir ci-dessus) offrent désormais une image plus complexe qu'il faudra préciser ces prochaines années. La phase ancienne du Magdalénien moyen est marquée par une diversification des équipements lithiques et osseux qui s'est traduite par une mosaïque de traditions 
ou faciès. Toutefois, une réévaluation de plusieurs séries tend à montrer qu'au delà de la présence-absence de certains marqueurs, de fortes tendances techno-typologiques unifient cette diversité (Langlais et al., soumis). L'ensemble supérieur des fouilles Trécolle livre un complexe lithique comparable à d'autres séries à lamelles scalènes du Sud-Ouest français qui semble se distinguer de séries à lamelles à dos tronquées. L'industrie osseuse présente des ressemblances avec la couche inférieure A de Blanchard, notamment dans les armatures (Blanchard et al., 1972 pl. II $n^{\circ} 1$ et pl. IV n $\left.{ }^{\circ} 1\right)$. En revanche, les quelques objets décorés de l'ensemble supérieur de Trécolle, à motifs de croisillons ou de zigzag, évoquent davantage des pièces de la couche $\mathrm{B}$ (fig. 14, $\mathrm{n}^{\text {os }}$ 6-8) mais aussi du Martinet (Le Tensorer, 1981 fig. 178). Un nouveau morphotype, la « pointe à base en languette », signe une particularité connue pour le moment uniquement à Gandil mais les recherches sur cet objet débutent à peine. De plus, si l'on note dans l'ensemble du gisement l'absence de pointes à biseau simple de type Lussac-Angles (Averbouh et Pétillon, 2013), certaines pointes à biseau double et certains décors sur industrie osseuse provenant des fouilles Mirande ou de la couche supérieure B du grand abri (Blanchard et al., 1972 fig. 12) ne dépareraient pas dans le « Magdalénien à navettes » (Allain et al., 1985). Enfin, la sépulture de la terrasse supérieure s'intègre parfaitement dans le groupe de sépultures primaires de cette période, telles Chancelade, Laugerie-Basse et Lafaye (Gambier et al., 2000; fig. 30) et peut-être El Miron en Espagne (Straus et al., 2011).

En définitive, la séquence Trécolle, si ce n'est l'ensemble du gisement de $\mathrm{SG}$, constitue un témoignage privilégié pour discuter à la fois dans le temps et dans l'espace des premiers pas du Magdalénien. Cette période se marque par une vaste géographie culturelle imbriquant des comportements et des objets communs avec des éléments originaux (Sécher, thèse en cours). Ces nouvelles discussions, au cœur du débat sur la structuration du Magdalénien à cette période (synchronie de groupes différents ou phasage interne?), ouvrent des pistes de recherche multiples qui nécessiteront sans doute un élargissement européen des comparaisons (de la Pologne aux Cantabres) mais également la poursuite de cet effort de réévaluation des collections anciennes.

Remerciements : Ce travail collectif a été mené grâce au soutien financier de l'Agence nationale de la recherche (ANR) dans le cadre du projet « Magdatis » (2011 BSH3 0005). Les auteurs remercient le laboratoire PACEA pour le financement de dates ARTEMIS, la fondation Fyssen, le musée national de Préhistoire des Eyzies, notamment J.-J. Cleyet-Merle et S. Madeleine, et la Société d'histoire et d'archéologie du Libournais pour l'accès aux collections Blanchard et Mirande. Nous remercions également D. Armand pour son aide lors du prélèvement des échantillons ainsi que l'équipe du laboratoire ORAU pour la qualité et l'efficacité de leur travail sur les datations. Nous remercions également B. Valentin et le second rapporteur anonyme pour leurs remarques constructives ainsi que L. Klaric et M. Sauvage pour leur travail éditorial.

\section{NOTES}

(1) Stratigraphie formée par les seuls éléments archéologiques pouvant être indépendante des attributions de couches affectées lors des fouilles.

(2) Travail qui devrait être précisé par des tests systématiques de remontages lithiques et osseux. Notons en L21 qu'un lot de pièces marquées $\mathrm{C} 1 / \mathrm{C} 2$ par le fouilleur et associant des marqueurs des ensembles inférieur et supérieur ont été attribuées à l'ensemble médian, lui conférant une morphologie emboîtée.

(3) Selon D. Stordeur-Yedid, « les plus grandes aiguilles, de 80 à $170 \mathrm{~mm}$, viennent presque toutes des couches anciennes du Placard (7 objets sur 10). Toutes les aiguilles qui mesurent plus de $100 \mathrm{~mm}$ ont été fabriquées au Placard dans une période qui va du Magdalénien I à III (sauf une aiguille de La Madeleine) » (Stordeur-Yedid, 1979, p. 185-186). L'auteur n'indique cependant pas si ces grandes aiguilles sont réalisées préférentiellement en bois de cervidé.

(4) Plus des $2 / 3$ des fragments de diaphyse sont de petite taille : moins de la moitié de la circonférence complète et moins d'un quart de la longueur initiale de l'os.

(5) Une canine de loup avait été collée par erreur dans une hémi-mandibule appartenant à un autre individu.

(6) Sans la prise en compte des fragments de bois, le renne ne représente que $15,4 \%$ des restes déterminés dans l'ensemble inférieur et 9,6\% dans l'ensemble supérieur.

(7) Indice de Simpson : $1 / \Sigma \mathrm{p}_{\mathrm{i}}{ }^{2}$ avec $\mathrm{p}_{\mathrm{i}}=\mathrm{NRD}_{\mathrm{i}} / \mathrm{NRD}$. Cet indice reflète le degré de dominance d'un taxon particulier au sein d'un cortège faunique. Plus l'indice est proche de 1 , plus le spectre est spécialisé.

(8) Cette surface est d'autant plus faible que seuls trois carrés ont livré un matériel conséquent.

(9) Pour les méthodes d'estimation d'âge de l'antilope saïga, voir Costamagno, 2001.

(10) Une courbe en L correspond à un profil d'âge d'une population vivante stable.

(11) Les études cémentochronologiques ont été réalisées par E. Pubert (in Costamagno 1999) puis reprises par W. Rendu (2007).

(12) Dans l'ensemble inférieur, le NMI à partir des restes osseux est de 16. Même en considérant que tous les fragments de côtes de taille moyenne sont de l'antilope saïga, chaque côte en moyenne ne devrait pas s'être fragmentée en plus de 3 morceaux pour que le nombre de fragments de côtes soit équivalent au nombre d'antilopes saïga. Or, près de $80 \%$ des fragments de côtes font moins de $2 \mathrm{~cm}(93 \%$ moins de $3 \mathrm{~cm}$ ).

(13) Pour les côtes, les mêmes remarques peuvent être réitérées : si toutes les côtes des 29 carcasses d'antilopes étaient présentes, il faudrait un NMI de 696 côtes. Ce nombre est à peine inférieur au nombre de fragments de côtes d'ongulés de petite et moyenne taille $(\mathrm{NR}=837)$ dont plus de $89 \%$ sont inférieurs à $2 \mathrm{~cm}$.

(14) Dans l'ensemble inférieur, plus d'un tiers des restes de rennes sont des fragments de bois (ont été pris en compte tous les fragments même s'ils ne comportent pas de critères diagnostiques); dans l'ensemble supérieur, ces pièces représentent un quart des vestiges de rennes.

(15) Si la peau est récupérée dans son entier, les incisions longitudinales de la peau des pattes doivent être réalisées sur la face interne des os des membres. Ces incisions qui rejoignent alors l'incision ventrale permettent d'obtenir une 
peau de grande surface. Si, au contraire, la peau des pattes est récupérée séparément de celle du tronc en pratiquant une incision circulaire non détectable sur le matériel archéologique à proximité du tronc, les incisions longitudinales peuvent alors être pratiquées sur n'importe quelle face.

(16) S'il est possible pour nous d'évaluer la fiabilité physico-chimique et stratigraphique des échantillons datés dans le cadre du projet « Magdatis », nous ne pouvons pas en dire de même pour la plupart des datations publiées, car les renseignements nécessaires à cette évaluation ne sont souvent pas présentés. Par exemple, ORAU ne trouve pas fiables les datations faites sur $<5 \mathrm{mg}$ de collagène ou ayant un taux $<$ $1 \%$ de collagène et ne datent pas d'habitude ceux ayant un taux d'azote $<0.76 \%$ (Brock et al., 2010). Ces critères (ou d'autres qui leur sont proches) ne sont pas forcément utilisés par d'autres laboratoires.

\section{RÉFÉRENCES BIBLIOGRAPHIQUES}

AdACHI T. (2000) - Études des industries magdaléniennes de Combe-Cullier (Lot), une approche typologique, lithologique et technologique, mémoire de DEA, université Bordeaux 1,2 vol.

Airvaux J., Brou L., Primault J. (2012) - Les outils sur lames tronquées amincies du Magdalénien moyen de LussacAngles, Préhistoire du Sud-Ouest, 20, 2, p. 143-178.

Allain J., Desbrosse R., KozŁowski J. K., Rigaud A. (1985) - Le Magdalénien à navettes, Gallia Préhistoire, 28, p. 37-124.

Altuna J. (2004) - Estudio biométrico de Vulpes vulpes L. y Alopex lagopus L. Contribución a su diferenciación en los yacimientos paleolíticos cantábricos, Munibe, 56, p. 45-59.

Andrews P., CooK J. (1985) - Natural modifications to bones in a temperate setting, Man, 20, p. 675-691.

Astier A. (2014) - Comportements techniques au Magdalénien moyen sur le site du Taillis des Coteaux (Vienne). Analyse technologique de l'industrie lithique : niveau EG-IIg, mémoire de master I, université Toulouse II, $201 \mathrm{p}$.

Averboun A., Pétillon J.-M. (2013) - L'industrie osseuse du Solutréen au Magdalénien moyen dans le Bassin parisien, in P. Bodu, L. Chemana, L. Klaric, L. Mevel, S. Soriano et N. Teyssandier (dir.), Le Paléolithique supérieur ancien de l'Europe du Nord-Ouest, actes du colloque (Sens, 15-18 avril 2009), Paris, Société préhistorique française (Mémoire, 56), p. 143-158.

Bannikov A. G., dir. (1967) - Biology of the Saiga, Jérusalem, Israel Program for Scientific Translation, $252 \mathrm{p}$.

Barshay-Szmidt C., Pétillon J.-M., Costamagno S., Laroulandie V., Langlais M., Mallye J.-B., HenryGambier D., Boudadi-Maligne M., Kuntz D. (en prép.) - The Chronology of the Middle and Late Magdalenian in the Western Aquitaine/Pyrenean Region of France (ca. 19-14 Ky cal BP). New Extensive Focused AMS ${ }^{14} \mathrm{C}$ Dating of the MAGDATIS Project and Overall Trends, Quaternary International.

Bartram L. E., Marean C. W. (1999) - Explaining the 'Klasies Patterns': Kua Ethnoarchaeology, the Die Kelders Middle Stone Age Archaeofauna, Long Bone Fragmentation, and Carnivore Ravaging, Journal of Archaeological Science, 26, p. 9-29.

BaziLe F. (2006) - Datations du site de Fontgrasse (Vers-Pontdu-Gard, Gard). Implications sur la phase ancienne du Magdalénien en France méditerranéenne, Bulletin de la Société préhistorique française, 103, 3, p. 597-602.
Bazile F., Monnet-Bazile C. (2000) - Le Magdalénien et 1'Après-Magdalénien en Languedoc oriental, in G. Pion (dir.), Le Paléolithique supérieur récent: nouvelles données sur le peuplement et l'environnement, actes de table ronde (Chambéry, 1999), Paris, Société préhistorique française (Mémoire, 28), p. 127-145.

Behrensmeyer A. K. (1978) - Taphonomic and Ecologic Information from Bone Weathering, Paleobiology, 4, p. 150-162.

Binford L. R. (1981) - Bones: Ancient Men and Modern Myths, New York, Academic Press, 320 p.

BlanCHARD R. (1935) - Découverte d'un squelette humain à Saint-Germain-la-Rivière, Revue historique et archéologique du Libournais, 9, p. 11-18.

Blanchard R., Peyrony D., Vallois H. V. (1972) - Le gisement et le squelette de Saint-Germain-la Rivière, Paris, Masson (Archives de l'Institut de paléontologie humaine, 34), $115 \mathrm{p}$.

Blumenschine R. J., Marean C. W., Capaldo S. D. (1996) Blind Tests of Interanalyst Correspondence and Accuracy in the Identification of Cut-marks, Percussion Marks and Carnivore Tooth Marks on Bone Surface, Journal of Archaeological Science, 23, p. 493-508.

Bodu P., Chehmana L., Debout G. (2007) - Le Badegoulien de la moitié nord de la France, Bulletin de la Société préhistorique française, 104, 4, p. 661-679.

Boudadi-Maligne M. (2010) - Les Canis pléistocènes du Sud de la France : approche biosystématique, évolutive et biochronologique, thèse de doctorat, université Bordeaux 1, $451 \mathrm{p}$.

Bourdier C. (2010) - Le Magdalénien moyen en PoitouCharentes : une expression symbolique propre, in J. Buisson-Catil et J. Primault (dir.), Préhistoire entre Charente et Vienne. Hommes et sociétés du Paléolithique, Chauvigny, Association des publications chauvignoises (Mémoire, 38), p. 363-382.

Bourdier C., Chehmana L., Pétillon J.-M., Valladas H. (2014a) - L'abri-sous-roche orné de Reverdit (Sergeac, Dordogne) : l'apport d'une approche pluridisciplinaire à l'élaboration d'un nouveau cadre chronoculturel, in J. Jaubert, N. Fourment et P. Depaepe (dir.), Transitions, ruptures et continuité en Préhistoire, 2. Paléolithique et Mésolithique, actes du XXVII ${ }^{e}$ Congrès préhistorique de France (Bordeaux - Les Eyzies, juin 2010), Paris, Société préhistorique française, p. 431-447.

Bourdier C., Pétillon J.-M., Chehmana L., Valladas H. (2014b) - Contexte archéologique des dispositifs parié- 
taux de Reverdit et de Cap-Blanc : nouvelles données, in P. Paillet (dir.), Les arts de la Préhistoire : micro-analyses, mises en contextes et conservation, Les Eyzies-de-Tayac, SAMRA (nº spécial de Paléo), p. 285-294.

Bouvier J.-M., Trécolle G. (1966) - La frise gravée de SaintGermain-la-Rivière, L'Anthropologie, 70, p. 535-540

Brock F., Higham T., Ditchfield P., Bronk Ramsey C. (2010) - Current Pretreatment Methods for AMS Radiocarbon Dating at the Oxford Radiocarbon Accelerator Unit (ORAU), Radiocarbon, 52, 1, p. 103-112.

Bronk Ramsey C., Lee S. (2013) - Recent and Planned Developments of the Program OxCal, Radiocarbon, 55, 2-3, p. 720-730.

Caux S. (2014) - A New Piece within a Larger Puzzle: Characterisation of 'Grain de Mil' Flint from the CharenteMaritime, a Central Resource for the Reconstruction of Palaeolithic Territories in South-western France, in A. Garcia, J. Garcia, A. Maximiano et J. Rios-Garaizar (dir.), Debating spatial archaeology, actes de la table ronde internationale (Santander, 8-9 juin 2012), Santander, IIIPC, p. 143-152.

Cazals N. (2005) - Le début du Magdalénien de part et d'autre des Pyrénées. Quelques réflexions au travers des techniques de taille et des modes d'exploitation des ressources, in J. Jaubert et M. Barbaza (dir.), Territoires, déplacements, mobilité, échanges durant la Préhistoire, actes du $126^{\mathrm{e}}$ Congrès du CTHS (Toulouse, 2001), Paris, CTHS, p. 295-309.

Chauvière F.-X., Rigaud A. (2005) - Les « sagaies » à « base raccourcie » ou les avatars de la typologie : du technique au " non-fonctionnel » dans le Magdalénien à navettes de la Garenne (Saint-Marcel, Indre), in V. Dujardin (dir.), Industrie osseuse et parures du Solutréen au Magdalénien en Europe, Paris, Société préhistorique française (Mémoire, 39), p. 233-242.

Chauvière F.-X., Rigaud A. (2008) - Le travail du bois de renne à la Garenne (Saint-Marcel, Indre) : entre conceptions préhistoriennes et techniques magdaléniennes, ou comment séparer ébauches et déchets des pointes vraies? Préhistoire du Sud-Ouest, 16, p. 173-183.

Chauviré C. (1965) - Les oiseaux du gisement magdalénien du Morin (Gironde), Actes du quatre-vingt-neuvième Congrès national des sociétés savantes (Lyon, 1964), section d'archéologie, Paris, Bibliothèque nationale, p. 255-266.

Cheynier A., Bouyssonie J. (1955) - Chancelade, abri de Raymonden, Bulletin de la Société historique et archéologique du Périgord, 82, p.172-188.

Cheynier A. (1965) - Les têtes de brochet, fossiles directeurs du Saint-Germien (Proto-Magdalénien IIb), Bulletin de la Société préhistorique française, comptes rendus des séances mensuelles, 62, 9, p. 315-323.

Costamagno S. (1999) - Stratégies de chasse et fonction des sites au Magdalénien dans le Sud de la France, thèse de doctorat, université Bordeaux I, 495 p.

Costamagno S. (2001) - Exploitation de l'antilope saïga au Magdalénien en Aquitaine : méthodes d'étude et applications archéologiques, Paléo, 13, p. 111-127.

Costamagno S. (2002) - Laboratory Taphonomy. Material Loss and Skeletal Part Profiles: The example of Saint-
Germain-la-Rivière (Gironde, France), Archaeometry, 44, p. $495-504$.

Costamagno S. (2012) - Des stries de boucherie au soussystème technique d'exploitation de l'animal : apports de l'approche expérimentale, mémoire d'habilitation à diriger des recherches université Bordeaux 1, 146 p.

Costamagno S., Rigaud J.-P. (2014) - L'exploitation de la graisse au Paléolithique, in S. Costamagno (dir.), Histoire de l'alimentation humaine : entre choix et contraintes, actes du 138 Congrès du CTHS (Rennes 22-26 avril 2013), Paris, CTHS, p. 134-152.

Costamagno S., Théry-Parisot I., Castel J.-C., Brugal J.-P. (2009) - Combustible ou non? analyse multifactorielle et modèles explicatifs sur les ossements brûlés paléolithiques, in I. Théry-Parisot, S. Costamagno et A. Henry (dir.), Gestion des combustibles au Paléolithique et au Mésolithique : nouveaux outils, nouvelles interprétations, actes du XV Congrès de 1'IUSPP (Lisbonne, 4-9 septembre 2006), Oxford, Archeopress (BAR, International Series 1914), p. 69-84.

Coulonges L. (1956) - Les industries à lamelles triangulaires du Paléolithique supérieur dites «magdaléniennes II », Bulletin de la Société d'études et de recherches préhistoriques des Eyzies, 6, p. 1-6.

Cupillard C., Welté A. C. (2006) - Le Magdalénien de la grotte Grappin à Arlay (Jura, France) : nouveaux regards, L'Anthropologie, 110, p. 624-683.

Delpech F. (1989) - Le temps de l'antilope saiga, in J.-P. Mohen (éd.), Le temps de la Préhistoire, Paris, Société préhistorique française; Dijon, Archeologia, p. 48-49.

Delpech F. (1972) - Fouilles de sauvetage dans le gisement magdalénien de Fongaban, commune de Saint-Émilion (Gironde), troisième partie : la faune, L'Anthropologie, 76, p. 615-629.

Despriee J., Tymula S., Rigaud A., dir. (2009) - Données récentes sur la Magdalénien de "La Garenne » (SaintMarcel, Indre) et la place du Magdalénien "à navettes " en Europe, actes du colloque (Argenton-sur-Creuse, octobre 2004), Saint-Marcel, ASSAAM, 256 p.

Drucker D., Henry-Gambier D., Lenoir M. (2005) - Alimentation humaine au cours du Magdalénien en Gironde d'après les teneurs en isotopes stables $\left({ }^{13} \mathrm{C},{ }^{15} \mathrm{~N}\right) \mathrm{du}$ collagène, Paléo, 17, p. 57-72.

Dubourg C. (1997) - Les expressions du naturalisme dans les arts graphiques du Paléolithique supérieur. Une vision du monde des chasseurs préhistoriques, thèse de doctorat, université Bordeaux I, $506 \mathrm{p}$.

Ducasse S., Langlais M. (2007) - Entre Badegoulien et Magdalénien inférieur, nos cœurs balancent... Approche critique des industries lithiques du Sud de la France et du Nord-Est espagnol entre 19000 et 16500 BP, Bulletin de la Société préhistorique française, 104, 4, p. 771-785.

DucAsSe S., Renard C., dir. (2013) - Sur l'évolution de l'organisation socio-économique des groupes humains entre la fin du Solutréen et les débuts du Magdalénien. Des Causses du Quercy aux contreforts pyrénéens entre 23500 et 18500 cal. $B P$, rapport annuel de programme collectif de recherche, service régional de l'Archéologie Midi-Pyrénées, Toulouse, $194 \mathrm{p}$. 
Ducasse S., Castel J.-C., Chauvière F.-X., Langlais M., Camus H., Morala A., Turq A. (2011) - Le Quercy au cœur du Dernier Maximum Glaciaire. La couche 4 du Petit Cloup Barrat et la question de la transition badegoulomagdalénienne, Paléo, 22, p. 101-154.

Fisher J. W. (1995) - Bone Surface Modifications in Zooarchaeology, Journal of Archaeological Method and Theory, 2, p. 7-68.

Fosse P., Laudet F., Selva N., Wajrak A. (2004) - Premières observations néotaphonomiques sur des assemblages osseux de Bialowieza (Nord-Est de la Pologne) : intérêts pour les gisements pléistocènes d'Europe, Paléo, 16, p. 91-116.

Fullola J. M., Mangado J., Tejero T., Petit M. A., Bergada M. M., Nadal J., Garcia-Arguelles P., Bartroli R., Mercadal O. (2012) - The Magdalenian in Catalonia (North-east Iberia), Quaternary International, 272-273, p. 55-74.

Gambier D., Valladas H., Tisnerat-Laborde N., Arnold M., Besson F. (2000) - Datation de vestiges humains présumés du Paléolithique supérieur par la méthode du carbone 14 en spectrométrie de masse par accélérateur, Paléo, 12, p. 201-212.

GARDE J.-A. (1934) - Inventaire des découvertes préhistoriques faites dans le Libournais (région de Sainte-Foy exceptée), Revue historique et archéologique du Libournais, 6, p. 55-64.

GAussen J. (1964) - La grotte ornée de Gabillou près Mussidan, Dordogne, Bordeaux, Delmas (Publication de l'Institut de Préhistoire de l'université de Bordeaux, mémoire 3), 68 p.

Gourichon L. (1994) - Les harfangs (Nyctea scandiaca L.) du gisement magdalénien du Morin (Gironde). Analyse taphonomique des restes d'un rapace nocturne chassé et exploité par les hommes préhistoriques, mémoire de maîtrise, université Lumière-Lyon 2, $180 \mathrm{p}$.

GrigGo C. (1997) - La faune magdalénienne de l'abri Gandil Bruniquel (Tarn-et-Garonne). Études paléontologique, taphonomique et archéozoologique, Paléo, 9, p. 279-294.

Henry-Gambier D., Bruzek J., Murail P., Houet F. (2002) - Révision du sexe du squelette de Saint-Germainla-Rivière (Gironde, France), Paléo, 14, p. 205-212.

KozŁowski S. K., Poltowicz-Bobak M., Bobak D., TerberGER T. (2012) - New Information from Maszycka Cave and the Late Glacial Recolonisation of Central Europe, Quaternary International, 272-273, p. 288-296.

LAdIER E. (2000) - Le Magdalénien ancien à lamelles à dos de l'abri Gandil à Bruniquel (Tarn-et-Garonne) : étude préliminaire de l'industrie de la c. 20, in G. Pion (dir.), Le Paléolithique supérieur récent : nouvelles données sur le peuplement et l'environnement, actes de la table ronde (Chambéry, 1999), Paris, Société préhistorique française (Mémoire, 28), p. 191-200.

LAdier E. (2002) - L'art mobilier de l'abri Gandil à Bruniquel (Tarn-et-Garonne, France), Bulletin de la Société archéologique et historique de Tarn-et-Garonne, 127, p. 7-24.

LANGlais M. (2007a) - Dynamiques culturelles des sociétés magdaléniennes dans leurs cadres environnementaux. Enquête sur 7000 ans d'évolution de leurs industries lithiques entre Rhône et Èbre, thèse de doctorat, universités de Toulouse - Le Mirail et Barcelone, $550 \mathrm{p}$.

LANGLAis M. (2007b) - Des identités qui se cherchent... Apports des industries lithiques à la question de l'origine du Magdalénien moyen dans le Sud-Ouest européen, Bulletin de la Société préhistorique française, 104, 4, p. 759-770.

Langlais M. (2008) - Chronologie et territoires au Magdalénien entre le Rhône et l'Èbre : l'exemple des armatures lithiques, in J.-M. Pétillon, M.-H. Dias-Merino, P. Cattelain, M. Honegger, C. Normand et N. Valdeyron (dir.), Recherches sur les armatures de projectile du Paléolithique supérieur au Néolithique, actes de la session C83 du $\mathrm{XV}^{\mathrm{e}}$ Congrès de l'IUSPP (Lisbonne, 4-9 septembre 2006) $=$ Palethnologie, 1, p. 220-249.

LANGLAIS M. (2010) - Les sociétés magdaléniennes de l'isthme pyrénéen, Paris, CTHS (Documents préhistoriques, 26), $337 \mathrm{p}$.

Langlais M., Ducasse S. (2013) - Badegoulien versus Magdalénien, II. Le Magdalénien inférieur quercinois, in M. Jarry, J.-P. Brugal et C. Ferrier (dir.), Modalités d'occupation et exploitation des milieux au Paléolithique dans le Sud-Ouest de la France : l'exemple du Quercy, actes de la session C67 $\mathrm{du} X \mathrm{~V}^{\mathrm{e}}$ congrès de l'IUSPP (Lisbonne, 4-9 septembre 2006), Les Ezyies de Tayac, SAMRA (Supplément à Paléo, 4), p. 379-394.

Langlais M., Ladier E., Chalard P., Jarry M., LacrampeCuyaubere F. (2007) - Aux origines du Magdalénien « classique »: les industries de la séquence inférieure de l'Abri Gandil (Bruniquel, Tarn-et-Garonne), Paléo, 19, p. 341-366.

Langlais M., Pétillon J.-M.,A[rchambault] de Beaune S., Cattelain P., Chauvière F.-X., Letourneux C., Szmidt C., Bellier C., Beukens R., David F. (2010) - Une occupation de la fin du dernier maximum glaciaire dans les Pyrénées : le Magdalénien inférieur de la grotte des Scilles (Lespugue, Haute-Garonne), Bulletin de la Société préhistorique française, 107, 1, p. 5-51.

Langlais M., Costamagno S., Laroulandie V., Pétillon J.-M., Discamps E., Mallye J.-B., Cochard D., Kuntz D. (2012) - The Evolution of Magdalenian Societies in SouthWest France Between 18,000 and 14,000 Cal. BP: Changing Environments, Changing Tool Kits, Quaternary International, 272-273, p. 138-149.

Langlais M., Laroulandie V., Pétillon J.-M., Mallye J.-B., Costamagno S. (2014) - Évolution des sociétés magdaléniennes dans le Sud-Ouest de la France entre 18000 et 14000 cal. BP : recomposition des environnements, reconfiguration des équipements, in J. Jaubert, N. Fourment et P. Depaepe (dir.), Transitions, ruptures et continuité en Préhistoire, 2. Paléolithique et Mésolithique, actes du XXVII ${ }^{e}$ Congrès préhistorique de France (Bordeaux - Les Eyzies, juin 2010), Paris, Société préhistorique française, p. 417-430.

Langlais M., Pétillon J.-M., Sécher A., Lenoir M. (soumis) - Les débuts du Magdalénien dans le Sud-Ouest français, témoignages croisés des équipements lithiques et osseux, in C. Bourdier, L. Chehmana, R. Malgarini et M. Poltowicz-Bobak (dir.), L'essor du Magdalénien : aspects culturels, symboliques et techniques des faciès à navettes et à 
Lussac-Angles, actes de la séance de la Société préhistorique française (Besançon, 17-19 octobre 2013), Paris, Société préhistorique française.

Laroulandie V. (2000) - Taphonomie et archéozoologie des oiseaux en grotte : applications aux sites paléolithiques $d u$ Bois-Ragot (Vienne), Combe Saunière (Dordogne) et de la Vache (Ariège), thèse de doctorat, université Bordeaux $1,396 \mathrm{p}$.

Le Brun-Ricalens F., Brou L. (2003) - Burins carénésnucléus à lamelles : identification d'une chaîne opératoire particulière à Thèmes (Yonne) et implications, Bulletin de la Société préhistorique française, 100, 1, p. 67-83.

Lenorr M. (1983) - Le Paléolithique des basses vallées de la Dordogne et de la Garonne, thèse de doctorat d'État, université Bordeaux I, 702 p.

LeNoIR M. (1996) - Saint-Germain-la-Rivière - Pillebourse, Bilan scientifique, service régional de l'archéologie de la région Aquitaine, Bordeaux, p. 68.

Lenolr M. (2000) - La Préhistoire ancienne en Gironde : apports des recherches récentes, Gallia Préhistoire, 42, p. $57-84$.

Lenoir M., Marmier F., Trécolle G. (1991) - Données nouvelles sur les industries de Saint-Germain-la-Rivière (Gironde), in 25 ans d'études technologiques en Préhistoire, actes des $\mathrm{XI}^{\mathrm{e}}$ Rencontres internationales d'archéologie et d'histoire (Antibes, 18-20 octobre 1990), Juan-les-Pins, APDCA, p. 245-254.

Lenoir M., Marmier F., Trécolle G. (1994) - Le gisement magdalénien de Saint-Germain-la-Rivière (Gironde) : données anciennes et acquis récents, Revue archéologique de Bordeaux, 85, p. 39-72.

Lenoir M., Marmier F., Trécolle G. (1995) - Le gisement paléolithique de Saint-Germain-la-Rivière, Société d'anthropologie du Sud-Ouest, 30, p. 139-153.

Lloveras, L., Moreno García M., Nadal, J. (2009) - Butchery, Cooking and Human Consumption Marks on Rabbit (Oryctolagus cuniculus) Bones: An Experimental Study, Journal of Taphonomy, 7, 2-3, p. 179-201.

Leroy-Prost C. (2008) - L'industrie sur matières dures animales, in A. Glory, B. Delluc et G. Delluc (dir.), Les recherches à Lascaux (1952-1963), Paris, CNRS (suppléments à Gallia Préhistoire, 39), p. 119-166.

Le Tensorer J.-M. (1981) - Le Paléolithique de l'Agenais, Paris, CNRS (Cahiers du Quaternaire, 3), 528 p.

Lyman R. L. (1994) - Vertebrate Taphonomy, Cambridge, Cambridge University Press, 524 p.

Mallye J.-B. (2011) - Réflexion sur le dépouillement des petits carnivores en contexte archéologique : apport de l'expérimentation, Archaeofauna, 20, p. 7-25.

Mallye J.-B., Costamagno S., Laroulandie V., Beauval C. (2009) - Impact des processus périglaciaires sur la préservation des ossements, Les Nouvelles de l'Archéologie, 118, p. 26-31.

Marean C. W., Frey J. (1997) - Animal Bones from Caves to Cities: Reverse Utility Curves as Methodological Artifacts, American Antiquity, 62, p. 698-711.
Marmier F., Trecolle G. (1973) - Réutilisations successives d'un bâton percé dans le Magdalénien supérieur de SaintGermain-la-Rivière, L'Anthropologie, 77, 7-8, p. 767-770.

Martin F. (2013) - Étude archéozoologique de la couche $3 d u$ gisement de Fongaban (Saint-Émilion, Gironde) : un cas de chasse spécialisée au bison au cours du Magdalénien supérieur dans le Sud-Ouest de la France, mémoire de master 1, université Toulouse 2 - Le Mirail, 158 p.

MASSET C. (2014) - L'apprentissage de la fracturation osseuse, mémoire de master 2, université de Bordeaux, $74 \mathrm{p}$.

Mirande H., Lépront R. (1933) - Le gisement de SaintGermain-la-Rivière (sa découverte), Revue historique et archéologique du Libournais, 1, p. 15-18.

Morel P., Müller W. (1997) - Hauterive-Champréveyres Un campement magdalénien au bord du lac de Neuchâtel. Étude archéozoologique, Neuchâtel, musée cantonal d'Archéologie, $149 \mathrm{p}$.

NilsSen P. J. (2000) - An Actualistic Butchery Study in South Africa and its Implications for Reconstructing Hominid Strategies of Carcass Acquisition and Butchery in the Upper Pleistocene and Plio-Pleistocene, Le Cap, University of Cape Town, $649 \mathrm{p}$.

Ouzrit L. (1986) - Recherches sur les faunes du Dryas ancien en Gironde. Le gisement de Saint-Germain-la-Rivière, thèse de doctorat, université Bordeaux 1, 147 p.

Peyrony D. (1936) - Le Magdalénien à triangles scalènes, L'Anthropologie, 46, p. 363-366.

Peyrony D., Peyrony E. (1938) - Laugerie-Haute, près des Eyzies (Dordogne), Paris, Masson (Archives de l'Institut de paléontologie humaine, 19), $84 \mathrm{p}$.

PéTILlon J.-M. (2006) - Note méthodologique sur l'étude de l'industrie osseuse : la recherche systématique de raccords, Bulletin de la Société préhistorique française, 104, p. 175179.

Pétillon J.-M., Ducasse S. (2012) - From Flakes to Grooves: a Technical Shift in Antlerworking During the Last Glacial Maximum in Southwest France, Journal of Human Evolution, 62, p. 435-465.

Pétillon J.-M., Langlais M., A[rchambault] de Beaune S., Beukens R., Chauvière F.-X., David F., LetourNeux C., Szmidt C. (2008) - Le Magdalénien de la grotte des Scilles (Lespugue, Haute-Garonne). Premiers résultats de l'étude pluridisciplinaire de la collection Saint-Périer, Antiquités Nationales, 39, p. 57-71.

Poplin F. (1976a) - Les grands vertébrés de Gönnersdorf. Fouilles 1968, Wiesbaden, F. Steiner, 212 p.

Poplin F. (1976b) - À propos du nombre de restes et du nombre d'individus dans les échantillons d'ossements, Cahier $d u$ Centre de recherches préhistoriques, 5, p. 61-74.

PRAT F. (1968) - Recherches sur les équidés pléistocènes en France, thèse de doctorat, université Bordeaux 1, 3 vol., $700 \mathrm{p}$.

Primault J., Gabilleau J., Brou L., Langlais M., Guérin S., et collab. (2007) - Le Magdalénien inférieur à microlamelles à dos de la grotte du Taillis des Coteaux à Antigny (Vienne, France), Bulletin de la Société préhistorique française, 104, 1, p. 5-30. 
Primault J. avec la collaboration de Berthet A.-L., Brou L., Delfour G., Gabilleau J., Griggo C., Guerin S., Henry-Gambier D., Houmard C., Jeannet M., Lacrampe-Cuyaubere F., Langlais M., Laroulandie V., Liard M., Liolios D., Lompre A., Lucquin A., Mistrot V., Rambaud D., Schmitt A., Soler L., TaboRIN Y., VISSAC C. (2010) - La grotte du Taillis-des-Coteaux à Antigny (Vienne), in J. Buisson-Catil et J. Primault (dir.), Préhistoire entre Vienne et Charente, hommes et sociétés $d u$ Paléolithique, Chauvigny, Association des publications chauvinoises (Mémoire, 38), p. 271-293.

Rambaud D., Laroulandie V., Primault J., Bearez, P. (2011) - Les poissons et les oiseaux du Taillis des Coteaux (Antigny, Vienne), niveaux magdaléniens : origine naturelle ou culturelle, in V. Laroulandie, J.-B. Mallye et C. Denys (éd.), Taphonomie des petits vertébrés : référentiels et transferts aux fossiles, actes de la table ronde du réseau thématique pluridisciplinaire « Taphonomie » (Talence, 20-21 octobre 2009), Oxford, Archeopress (BAR, International Series, S2269), p. 167-179.

Reimer P. J., Bard E., Bayliss A., Beck J. W., Blackwell P. G., Bronk Ramsey C., Buck C. E., Cheng H., Edwards R. L., Friedrich M., Grootes P. M., Guilderson T. P., Haflidason H., Hajdas I., Hatte C., Heaton T. J., Hoffmann D. L., Hogg A. G., Hughen K. A., KaISer K. F., Kromer B., Manning S. W., Niu M., Reimer R.W., Richards D. A., Scott E. M., Southon J. R., StafF R. A., Turney C. S. M., VAn der Plicht J. (2013) - Intcal13 and Marine13 Radiocarbon Age Calibration Curves 0-50,000 Years Cal. BP, Radiocarbon, 55, 4, p. 1869-1887.

RENDU W. (2007) - Planification des activités de subsistance au sein du territoire des derniers moustériens, thèse de doctorat, université Bordeaux 1, $363 \mathrm{p}$.

Rigaud J.-P. (1979) - À propos des industries magdaléniennes du Flageolet, in D. de Sonneville-Bordes (dir.), La fin des temps glaciaires en Europe. Chronostratigraphie et écologie des cultures du Paléolithique final, actes du colloque (Talence, 1977), Paris, CNRS, p. 467-469.

SÉCHER A. (2013) - La genèse du Magdalénien moyen dans le Sud-Ouest de la France. Apports de la série lithique du Roc de Marcamps 2 (Prignac-et-Marcamps, Gironde), mémoire de master 2, université Bordeaux 1, $80 \mathrm{p}$.

Serjeantson D. (2009) - Birds, Cambridge, Cambridge University Press, XXVI-496 p.

Simonnet R. avec la collaboration de Bon F., Bordes J.-G., Primault J. (2007) - Le silex « Grains de Mil ». Localisation des gîtes, in N. Cazals et X. Terradas (dir.), Frontières naturelles et frontières culturelles dans les Pyrénées préhistoriques, actes de la table ronde (Tarascon, Ariège, 2004), Santander, Publican Ediciones univ. de Cantabria, p. 101102.

SlotT-Möller R. (1988) - Contribution à l'étude paléontologique d'un gisement préhistorique : l'exemple du Roc de Marcamps (Gironde), mémoire de diplôme d'études supérieures de sciences naturelles, université Bordeaux 1, 173 p.

Sonneville-Bordes D. De (1960) - Le Paléolithique supérieur en Périgord, Bordeaux, Delmas, $558 \mathrm{p}$.

Soulier M.-C., Kuntz D., Lacarrière J., Castel J.-C. (2014) - Le renne comme ressource alimentaire : discussion entre pratiques actuelles et paléolithiques, in S. Costamagno (dir.), Histoire de l'alimentation humaine : entre choix et contraintes, actes du $138^{\mathrm{e}}$ Congrès du CTHS (Rennes, 22-26 avril 2013), Paris, CTHS, p. 153-169.

Speth J. D., Spielman K. A. (1983) - Energy Source, Protein Metabolism, and Hunter-gatherer Subsistence Strategies, Journal of Anthropological Archaeology, 2, p. 1-31.

Steele T. E., Weaver T. D. (2002) - The Modified Triangular Graph: a Refined Method for Comparing Mortality Profiles in Archaeological Samples, Journal of Archaeological Science, 29, p. 317-322.

STINER M. C. (1990) - The Use of Mortality Patterns in Archaeological Studies of Hominid Predatory Adaptations, Journal of Anthropological Archaeology, 9, p. 305-351.

Stordeur-Yedid D. (1979) - Les aiguilles à chas au Paléolithique, Paris, CNRS (suppléments à Gallia Préhistoire, 13), $215 \mathrm{p}$.

Straus L. G., González Morales M. R., Carretero J. M. (2011) - Lower Magdalenian Secondary Human Burial in El Miron Cave, Cantabria, Spain, Antiquity, 85, p. 1151-1164.

Szmidt C., Pétillon J.-M., Cattelain P., Normand C., Schwab C. (2009) - Premières dates radiocarbone pour le Magdalénien d'Isturitz (Pyrénées-Atlantiques), Bulletin de la Société préhistorique française, 106, 3, p. 588-592.

Val A., Mallye J.-B. (2011) - Small Carnivore Skining by Professionnals: Skeletal Effects and Implications for the European Upper Palaeolithic, Journal of Taphonomy, 9, 4, p. $221-243$

Vanhaeren M., D’Errico F. (2003) - Le mobilier funéraire de la Dame de Saint-Germain-la-Rivière (Gironde) et l'origine paléolithique des inégalités, Paléo, 15, p. 195-238.

VAufrey R. (1935) - L'homme magdalénien de Saint-Germainla-Rivière, L'Anthropologie, 45, p. 203-204.

Villa P., Mahieu E. (1991) - Breakage Patterns of Human Long Bones, Journal of Human Evolution, 21, p. 27-48.

White T. D. (1992) - Prehistoric Cannibalism at Mancos 5MTUMR-2346, Oxford, Princeton University Press, 462 p.

Mathieu Langlais

UMR 5199 «PACEA», Bordeaux mathieu.langlais@u-bordeaux.fr

Véronique LAROULANDIE

UMR 5199 « PACEA», Bordeaux veronique.laroulandie@u-bordeaux.fr

Sandrine Costamagno UMR 5608 « TRACES », Toulouse costamag@univ-tlse2.fr

Jean-Marc PÉtillon

UMR 5608 «TRACES», Toulouse petillon@univ-tlse2.fr 
Jean-Baptiste Mallye

UMR 5199 « PACEA », Bordeaux jean-baptiste.mallye@u-bordeaux.fr

François Lacrampe-Cuyaubère Société Archéosphère f.lacrampe@archeosphere.com

Myriam Boudadi-MaLigne UMR 5199 « PACEA », Bordeaux myriam.boudadi-maligne@u-bordeaux.fr

Carolyn BarshaY-SzMidT University of Pennsylvania Museum of Archaeology and Anthropology Toronto, Canada carolyn_szmidt22@yahoo.ca
Caroline MAsset

Étudiante en master 2, université de Bordeaux

UMR 5199 « PACEA », Bordeaux caroline.masset@yahoo.fr

Éric Pubert

UMR 5199 « PACEA», Bordeaux e.pubert@pacea.u-bordeaux1.fr

William RENDU

UMI 3199 « CIRHUS », New York University williamrendu@hotmail.fr

Michel LenoIr

Préhistorien retraité blackmike33@orange.fr 